\title{
MODELLING THE GENERATION OF THE COCHLEAR MICROPHONIC
}

BY

MOHAMMAD AYAT

A thesis

submitted to the Victoria University of Wellington in fulfilment of the requirements for the degree of

Doctor of Philosophy

in

Electronic and Computer System Engineering

2014 
Modelling the Generation of the Cochlear Microphonic

Mohammad Ayat

M.Sc., Sharif University of Technology, 2006

B. Sc., Sahand University of Technology, 2003

Supervisors:

Dr. Paul D. Teal

Dr. Mark McGuinness 


\begin{abstract}
The human ear is a remarkable sensory organ. A normal healthy human ear is able to process sounds covering a wide range of frequencies and intensities, while distinguishing between different components of complex sounds such as a musical chord.

In the last four decades, knowledge about the cochlea and the mechanisms involved in its operation has greatly increased, but many details about these mechanisms remain unresolved and disputed.

The cochlea has a vulnerable structure. Consequently, measuring and monitoring its mechanical and electrical activities even with contemporary devices is very difficult. Modelling can be used to fill gaps between those measurements that are feasible and actual cochlear function. Modelling techniques can also help to simplify complex cochlear operation to a tractable and comprehensible level while still reproducing certain behaviours of interest. Modelling therefore can play an essential role in developing a better understanding of the cochlea.

The Cochlear Microphonic (CM) is an electrical signal generated inside the cochlea in response to sound. This electrical signal reflects mechanical activity in the cochlea and the excitation processes involved in its generation. However, the difficulty of obtaining this signal and the simplicity of other methods such as otoacoustic emissions have discouraged the use of the cochlear microphonic as a tool for studying cochlear functions.

In this thesis, a model of the cochlea is presented which integrates both mechanical and electrical aspects, enabling the interaction between them to be investigated. The resulting model is then used to observe the effect of the cochlear amplifier on the CM. The results indicate that while the cochlear amplifier significantly
\end{abstract}


amplifies the basilar membrane displacement, the effect on the $\mathrm{CM}$ is less significant. Both of these indications agree with previous physiological findings. A novel modelling approach is used to investigate the tuning discrepancy between basilar membrane and CM tuning curves. The results suggest that this discrepancy is primarily due to transversal phase cancellation in the outer hair cell rather than longitudinal phase cancellation along the basilar membrane. In addition, the results of the model suggest that spontaneous cochlear microphonic should exist in the cochlea. The existence of this spontaneous electrical signal has not yet been reported. 


\section{Acknowledgements}

This study would not have been possible without the help and support of many people.

First and the foremost I wish to express my deepest gratitude to Dr Paul Teal, my supervisor, for welcoming me as his student, for sharing his knowledge and his idea, for his constant support and availability during my PhD work. I would like to thank Dr Mark McGuinness, for all his support and encouragement throughout my work.

Special thanks go to my officemate Ahmed Sheik Deeb and my friend Reza Daj. They are amazing friends and unconditionally helped me during my $\mathrm{PhD}$ study. I would also like to extend my thanks to my fellow $\mathrm{PhD}$ friends in our Communications and Signal Processing (CaSP) Group, especially Praveen Choppala, Asim Masood for their help. I am also grateful to the examination committee for their time, their valuable comments and suggestions to improve the quality of my thesis.

I am deeply indebted to all my friends in Wellington who have supported me over the last four years: Phil Hamil, Davoud Zare, Mahroo Falah, Babette Polly, Mohsen Joshanloo, Mazyar Mahmoudabadi and many others.

I wish to express my warmest thanks to my parents Hossein and Shahnaz, my brother and sisters Masoud, Moloud and Marzieh for the encouragement and the endless love.

Last but not least and above all, I am very very thankful to my beloved wife Mojdeh for all her love, patient, support and for encouraging me to keep working. She believed in me when I did not. She was with me in every difficulty and she was strong when I was weak. Thanks again Mojdeh.

The financial support provided by the Marsden Fund, administered by the Royal Society of New Zealand and Victoria University of Wellington is also much appreciated. 


\section{Contents}

1 Introduction 1

1.1 Modelling ...................... 2

1.2 Cochlear Microphonic . . . . . . . . . . . . . 3

1.3 Contributions and Thesis Overview . . . . . . . . . . . 4

1.4 Publications ...................... 7

2 Background 9

2.1 Anatomy and Physiology of the Human Ear . . . . . . . . . . 9

2.1.1 The Human Cochlea . . . . . . . . . . . . . . . 11

2.2 Hair cells . . . . . . . . . . . . . . . . . . . . 12

2.3 Active Processes and Cochlear Amplifier . . . . . . . . . . . 17

2.4 What is the Source of the Active Process? . . . . . . . . . . 21

2.4.1 Hair Bundle Motility . . . . . . . . . . . . . . 21

2.4 .2 Somatic Motility . . . . . . . . . . . . . 22

2.5 Electrical Activities Inside the Cochlea . . . . . . . . . . . . . 22

2.5.1 Cochlear Microphonic . . . . . . . . . . . 23

2.5.2 A Concise History of the Cochlear Microphonic . . . . . 24 
2.5.3 Applications of the Cochlear Microphonic . . . . . . . . 25

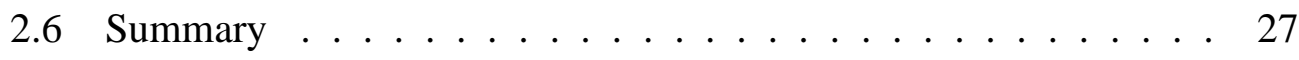

3 Modelling $\quad 29$

3.1 Introduction . . . . . . . . . . . . . . . . . . 29

3.2 Models of Cochlear Mechanics . . . . . . . . . . . . . . . 31

3.2.1 Mechanical properties of the cochlea . . . . . . . 31

3.2.2 Cochlear Macromechanics ............. 31

3.2.3 Cochlear Micromechanics ... . . . . . . . . 33

3.3 Models Of Electrical Coupling . . . . . . . . . . . . . 34

3.3.1 Electrical Properties of the Cochlea . . . . . . . . . 34

3.3.2 Electrical Modelling of the Organ of Corti . . . . . . . 36

3.3.3 Cochlear Microphonic . . . . . . . . . . . . 39

3.4 Modelling the Generation of the $\mathrm{CM} \ldots \ldots$. . . . . . . . 40

3.4.1 The Model of Liu and Neely (2010). . . . . . . . . . . . . 41

3.4.2 Electrical Lumped Model of The Organ of Corti . . . . 46

3.5 Summary . . . . . . . . . . . . . . . . . . 48

3.6 Contributions . . . . . . . . . . . . . . . . . . 49

4 Methods of Solution and Model Validation $\quad 51$

4.1 State Space Representation of the Model . . . . . . . . . . . . . . 52

4.2 Frequency Domain Analysis . . . . . . . . . . . . 57

4.2.1 Mechanical Responses . . . . . . . . . . . . . 58

4.2.2 Electrical Responses . . . . . . . . . . . . . 62

4.3 Time Domain Analysis . . . . . . . . . . . . . . . 65 
4.3.1 Response to a broadband Stimulus . . . . . . . . . . . 66

4.4 Effect of Cochlear Amplifier on the CM . . . . . . . . . 67

4.5 Summary ...................... 71

4.6 Contributions . . . . . . . . . . . . . . . 72

5 Cochlear Microphonic Broad Tuning Curves 73

5.1 Broadness of the CM Tuning Curves . . . . . . . . . . . . . 74

5.2 Effect of Longitudinal Phase Cancellation on the CM Tuning Curves 75

5.3 Effect of Transversal Phase Cancellation on the CM Tuning Curves 78

5.3.1 Longitudinal Resistors . . . . . . . . . . . . . . 79

5.3 .2 Discussion ................. 83

5.4 Summary . . . . . . . . . . . . . . . . . 84

5.5 Contribution .................... 85

$\begin{array}{llr}6 & \text { Spontaneous Cochlear Microphonic } & 87\end{array}$

6.1 Introduction . . . . . . . . . . . . . . . 87

6.2 Stability of the Proposed Model _ . . . . . . . . . . . . . 89

6.2.1 Effects of Irregularities on the Stability . . . . . . . . . 91

6.2.2 Time-domain Simulations of the Model Including Irregularities and the existence of SCOMIC . . 94

6.3 Summary and Conclusion . . . . . . . . . . . . . . . . . . 99

6.4 Contribution . . . . . . . . . . . . . . . 101

7 Summary, Conclusions and Suggestions for Further Work 103

7.1 Summary and Conclusions of Present Work . . . . . . . . . . 104

7.2 Limitations of the model . . . . . . . . . . . . . . . 107 
7.3 Future Work . . . . . . . . . . . . . . . . . . 108

$\begin{array}{ll}\text { A State space formulation } & 111\end{array}$

A.1 State Space Matrices . . . . . . . . . . . . . . 111

B Using Circuit Analogies for Analysis of Cochlear Models 119

B.1 Materials and Methods . . . . . . . . . . . . 120

B.1.1 Equivalent Circuit of the Sound Source and Middle Ear Models ..................... . 121

B.1.2 Equivalent Circuit of Cochlear Macromechanics . . . . 121

B.1.3 Equivalent Circuit of Cochlear Micromechanics . . . . . . 122

B.2 SPICE implementation . . . . . . . . . . . . 123

B.3 Discussion and Summary . . . . . . . . . . . . . 127

C Parameter Selection and Calibration 129

C.1 Mechanical Parameter Values . . . . . . . . . . . . . . . 130

C.2 Electrical Parameter Values . . . . . . . . . . . . . . . . 131

C.2.1 Longitudinal Resistors . . . . . . . . . . . . 131

C.2.2 Radial Electrical Elements . . . . . . . . . . . . 135

C.3 Calibration . . . . . . . . . . . . . . . 139 


\section{Glossary}

BM Basilar Membrane.

BP Best Place.

CAP Compound Action Potential.

CF Characteristic Frequency.

CM Cochlear Microphonic.

HB Hair Bundle.

IHC Inner Hair Cell.

MET Mechanoelectrical Transduction.

OAE Otoacoustic Emission.

OHC Outer Hair Cell.

PCM Proposed Cochlear microphonic Model.

RL Reticular Lamina.

SCOMIC Spontaneous COchlear MICrophonic.

SOAE Spontaneous Otacoustic Emission.

ix 
SP Summating Potential.

SPL Sound Pressure Level. 


\section{List of Symbols}

$A_{\mathbf{d}}$ The area of the earphone diaphragm.

$A_{\mathbf{e}}$ The area of the eardrum.

$A_{\mathbf{s}}$ The area of the stapes footplate.

$A$ Cochlear cross-sectional area.

$C_{3}$ Apical capacitance of the outer hair cell.

$C_{4}$ Basolateral capacitance of the outer hair cell.

$C_{\mathbf{g}}$ Gating capacitance of the outer hair cells.

$H$ Height of the scalae vestibuli and media (assumed constant).

$I_{\max }$ Maximum range of outer hair cell receptor current.

$K_{\mathbf{c}}$ Acoustic compliance.

$K_{\mathbf{d}}$ The stiffness of the earphone diaphragm.

$K_{\mathbf{i}}$ The stiffness of the incudo-stapedial joint.

$K_{\mathbf{m}}$ The stiffness of the eardrum-malleus-incus system.

$K_{\mathbf{r}}$ The stiffness of the round window.

$K_{\mathbf{S}}$ The stiffness of the stapes. 
$K$ The stiffness of the outer hair cell load impedance.

$L$ Length of the cochlea.

$M_{\mathbf{d}}$ The mass of the earphone diaphragm.

$M_{\mathbf{m}}$ The mass of the eardrum-malleus-incus system.

$M_{\mathbf{r}}$ The mass of the round window.

$M_{\mathrm{s}}$ The mass of the stapes.

$M$ The mass of the outer hair cell load impedance.

$P$ The fluid pressure difference between scala vestibuli and scala tympani.

$Q$ Charge accumulation that accompanies outer hair cell electromotility.

$R_{11}$ The resistances along the scala tympani.

$R_{1}$ The resistance between the scala vestibuli and the surrounding spiral ligament.

$R_{3}$ Apical resistance of the outer hair cell.

$R_{4}$ Basolateral resistance of the outer hair cell.

$R_{5}$ The resistance between the scala tympani and the surrounding spiral ligament.

$R_{6}$ The resistance between the scala media and the surrounding spiral ligament.

$R_{8}$ The resistances along the scala vestibuli.

$R_{9}$ The resistances along the scala media.

$R_{\mathbf{d}}$ The resistance of the earphone diaphragm.

$R_{\mathbf{i}}$ The resistance of the incudo-stapedial joint.

$R_{\mathbf{m}}$ The resistance of the eardrum-malleus-incus system.

$R_{\mathbf{r}}$ The resistance of the round window. 
$R_{\mathrm{S}}$ The resistance of the stapes.

$R$ The resistance of the outer hair cell load impedance.

$T$ Piezoelectric constant of the outer hair cells.

$U$ the cochlear fluid volume velocity along the $x$-direction..

$V_{\mathbf{H B}}$ Potential of the hair bundle.

$V_{\text {OHC }}$ Potential of outer hair cell membrane.

$V_{\text {sm }}$ Potential of the scala media.

$V_{\text {st }}$ Potential of the scala tympani.

$V_{\mathbf{s v}}$ Potential of the scala vestibuli.

$\alpha_{\mathbf{d}}$ The sensitivity of MET's to reticular lamina displacement.

$\alpha_{\mathbf{v}}$ The MET's sensitivity to reticular lamina velocity.

$\ddot{\xi}_{\mathbf{b}}$ The acceleration of the basilar membrane.

$\ddot{\xi}_{\text {o }}$ The acceleration of the outer hair cell.

$\dot{v}_{\mathbf{d}}$ The acceleration of the earphone diaphragm.

$\dot{\xi}_{\mathbf{b}}$ The velocity of the basilar membrane.

$\dot{\xi}_{\text {o }}$ The velocity of the outer hair cell.

$\dot{\xi}_{\text {r }}$ The velocity of the reticular lamina.

$f_{\text {OHC }}$ The force induced by outer hair cells electromotility.

$f(t)$ The stimulus force on the earphone diaphragm.

$g$ The malleus-incus lever ratio.

$k$ The stiffness of the basilar membrane per unit area. 
$m$ The mass of the basilar membrane per unit area.

$P_{\mathbf{d}}$ The pressure in the enclosed space of the ear canal.

$\rho$ The cochlear fluid density.

$r$ The resistance of the basilar membrane per unit area.

$v_{\mathbf{d}}$ The velocity of the earphone diaphragm.

$v_{\mathbf{S}}$ The velocity of the stapes.

$w$ The width of the basilar membrane.

$x_{\mathbf{d}}$ The displacement of the earphone diaphragm.

$\xi_{\mathbf{b}}$ The displacement of the basilar membrane.

$\xi_{0}$ The displacement of the outer hair cell.

$\xi_{\mathbf{r}}$ The displacement of the reticular lamina.

$x_{\mathbf{m}}$ The displacement of the malleus.

$x_{\mathbf{s}}$ The displacement of the stapes. 


\section{General comments}

- For clarity time-partial derivative sometimes are shown with dot as:

$$
\dot{x}=\frac{\partial}{\partial t} x
$$

or

$$
\ddot{x}=\frac{\partial^{2}}{\partial t^{2}} x
$$

- The proposed cochlear microphonic model, PCM, the model, our model, the proposed model, the proposed electromechanical model are used interchangeably throughout the thesis, and all refer to the proposed model.

- We have used Matlab ${ }^{\circledR}$ to implement the proposed model.

- All the work presented in this thesis has been performed solely by me, unless otherwise stated. Throughout the thesis, I refer to myself (sometimes with the reader) whenever the personal pronoun "we" is used. 


\section{Chapter 1}

\section{Introduction}

Despite great advances in hearing research, many details of the hearing mechanism still remain unclear. In mammals, sounds pass through different parts of the auditory system to reach a snail-shaped, fluid-filled organ known as the cochlea (Rhode, 1984; Robles and Ruggero, 2001; Kandel et al., 2012, Chapter 30). The cochlea is the central part of the auditory system which converts vibrations to a form comprehensible to the brain. The cochlea not only codes the sound into neural impulses but it also carries out the first tier analysis of the sound and classifies sound to different frequency bands and then sends corresponding information to the brain (Moller, 2006, Chapter 1).

The cochlea has a very sophisticated, complex and vulnerable structure. Consequently, measuring and monitoring its mechanical and electrical activities even with contemporary devices is very difficult and sometimes impossible. In addition, the results of these measurements even with our present-day understanding of cochlear function are hard to interpret (Wilson, 2008).

The cochlea includes electrical and mechanical parts which interact bidirectionally to convert vibrations to neural stimulation. These interactions produce both acoustical and electrical behaviours which can be measured with appropriate instrumentation. The focus of this thesis is on behaviours which can be observed 
electrically.

There are many neural activities in the auditory nerves and brainstem, but the cochlea itself also produces electrical signals. When the cochlea operates, in addition to action potentials of neural activities inside the cochlea, other bioelectric potentials can be observed by placing an electrode anywhere near (e.g., a convenient place for placing electrode near the cochlea in humans is the round window), on the surface or in the cochlea; one of these potentials is called the cochlear microphonic.

In the next chapter, it is shown that the cochlear microphonic is a reflection of cochlear mechanical activities, and hence can play a pivotal role in understanding cochlear function.

In general, electrical models of the cochlea can provide useful information and further our current knowledge of physiological and mechanical processes in the cochlea.

\subsection{Modelling}

The spectacular performance of the human cochlea which surpasses even the performance of the human visual system (Duke, 2002) clearly indicates that the cochlea is the most complex sensory organ in the human body (Moller, 2006, Chapter 1). Modelling techniques can help to simplify cochlear operation to a tractable and comprehensible level while still reproducing the behaviours of interest. The resulting model can be used to understand normal and abnormal function and test different hypotheses related to the cochlea. An acceptable biomedical model of an organ should agree with and explain certain relevant observations and behaviours. In addition, the model should allow specific predictions about that organ, which can be validated or invalidated by experiments or future observations.

Modelling has always been an inseparable and integral part of auditory research. 
From the time von Helmholtz described the ear as like a piano with an array of resonators each of which oscillates at a different frequency, until now, modelling has been gradually growing in importance to hearing research (Duke, 2002; Meddis and Lopez-Poveda, 2010). In recent years numerous auditory models have been suggested, revised, and discarded and some of them are still the subject of debate. Some of these models are reviewed in the following chapters. By using modelling techniques, hearing modellers try to classify auditory measurements and observations. They also try to explain phenomena which are not directly measurable and devise experiments to validate their model.

Researchers use models to improve the understanding of the cochlea from different perspectives. Electrical activities in mammalian cochleae are a significant part of the auditory sensing process. For investigating the properties and effects of these electrical activities, the cochlea can be considered as an electrical network of biological resistances, capacitances, voltage and current sources or simply an electrical model. Even though purely mechanical models of the cochlea can provide much information about the cochlea function and further our knowledge about this astonishing organ, incorporating a detailed electrical model of the cochlea can further our understanding of the cochlea considerably.

\subsection{Cochlear Microphonic}

One important but little appreciated electrical signal produced by the cochlea is the cochlear microphonic (Cheatham et al., 2011). The discovery of this electrical signal by Wever and Bray (1930a) and then the interpretation of it by Adrian (1931) provided a starting point for understanding electrical activities inside the cochlea (Ramsden, 2013). The cochlear microphonic is an important outcome of electrical activities in the cochlea which can be employed in clinical practice and auditory research (Zhang, 2013). However, in spite of the importance of the CM, the difficulty of obtaining this very small signal and uncertainty in its interpretation have meant that it is rarely used as an indication of cochlear 
performance, even after more than eighty years since its discovery (Teal et al., 2011; Cheatham et al., 2011). Several limited research and clinical applications of the CM are reviewed in the next chapter. These include assessing the mechanoelectrical transduction (MET) current of outer hair cells (Patuzzi and Moleirinho, 1998; Patuzzi et al., 1989; Patuzzi and O'Beirne, 1999), checking the biological effects of infrasound on the human auditory system (Salt and Hullar, 2010; Chen and Narins, 2012), diagnosing Ménière's disease (Norman et al., 1997), diagnosis of auditory neuropathy spectrum disorder (Roush, 2008) and examining the functionality of the ear objectively ${ }^{1}$ (Poch-Broto et al., 2009; Chertoff et al., 2012).

In clinical practice the cochlear microphonic is traditionally recorded invasively by transtympanic electrocochleography through electrodes placed on the round window or promontory of the cochlea (Atcherson and Stoody, 2012, Chapter 12); (He and Ren, 2013). Improvements in measurement techniques have also permitted the reliable non-invasive recording of this signal (Poch-Broto et al., 2009; Masood et al., 2012). In animal experiments, the CM can also be recorded by placing electrodes inside or on the surface of the cochlea (Honrubia and Ward, 1968; Liberman et al., 2002; Russell, 2008).

Modelling the generation of the cochlear microphonic can help to classify and examine current beliefs about the generation procedure of this signal and help to answer ambiguities about the interpretation of this bio-potential (Russell, 2008; Cheatham et al., 2011).

\subsection{Contributions and Thesis Overview}

The main goal of this doctoral work is to model the generation of the cochlear microphonic. This model is then used to explain several important characteristics

\footnotetext{
${ }^{1}$ The $\mathrm{CM}$ is useful particularly when screening the hearing of an infant or any person who cannot cooperate during behavioural (subjective) testing. In behavioural testing, the person will be told to raise his or her hand or push a button when they hear a sound.
} 
of the cochlear microphonic, and facilitate opportunities to increase understanding of mechanical and electrical interactions.

The next chapter provides the necessary background for this research. The major contributions of this research are presented in Chapters 3 to 6. The contributions are clearly highlighted at the end of each chapter. In the following, a brief overview of each chapter is given:

\section{Chapter 2: Background}

In this chapter, the physiology and anatomy of the human cochlea and hair cells are outlined. Cochlear interactions and relevant physical concepts are discussed. A historical review of the early works pertinent to this research is presented, and several applications of the CM are addressed.

\section{Chapter 3: Modelling}

This chapter begins with a comprehensive and informative literature review of cochlear mechanics and cochlear electrical modelling. This is then followed by a presentation of the proposed cochlear model for modelling the generation of the cochlear microphonic; the Proposed Cochlear microphonic Model (PCM).

\section{Chapter 4: Methods of Solution and Model Validation}

This chapter introduces the state-space formulism and its application to the PCM formalism and presents methods of implementing the model including an equivalent circuit representation. The validity of the model is then verified using the results of both frequency domain and time domain analyses and comparing them with available experimental data. The effect of the cochlear amplifier on the $\mathrm{CM}$ is also assessed using the results of the model in this chapter. 


\section{Chapter 5: Cochlear Microphonic Broad Tuning Curves}

An important phenomenon related to the $\mathrm{CM}$ that requires more investigation is the broadness of $\mathrm{CM}$ tuning curves. The $\mathrm{CM}$ measurements for different frequency and intensity stimuli show that unlike the sharp tuning curves of the Basilar Membrane (BM), the CM exhibits broad tuning curves (Honrubia and Ward, 1968; Dallos et al., 2005; Patuzzi, 1987; He et al., 2012a; Gelfand, 2010, Chapter 4). This question is thoroughly investigated in this chapter and original results are presented.

\section{Chapter 6: Spontaneous Cochlear Microphonic}

By using a sensitive microphone inside the ear canal, Kemp (1979) observed that human ear can emit spontaneous acoustic signals which are now referred to as Spontaneous Otacoustic Emissions (SOAEs) (Probst et al., 1991). We postulate the possibility of the existence of spontaneous cochlear microphonic. In this chapter we use the approach of Elliott et al. (2007), to demonstrate the model is capable of producing SOAEs. Consequently we predict the existence of measurable spontaneous cochlear microphonic. The existence of the spontaneous CM has not been previously reported.

\section{Chapter 7: Summary, Conclusions and Suggestions for Further Work}

In the last chapter, the findings of the current work are summarised. Some of the possible directions of future research are presented. Some open problems in current understanding of cochlea function are outlined for future studies. 


\section{Appendix A: State space formulation}

The details of the matrices of the state-space representation are reported in this appendix.

\section{Appendix B: Using Circuit Analogies for Analysis of Cochlear Models}

The procedure for converting the entire model (mechanical and electrical) to equivalent circuits is reported in this appendix. This procedure can be used as a framework to converting any one-dimensional cochlear model to equivalent electrical circuits.

\section{Appendix C: Parameter selection and Calibration}

Parameter selections and calibrations are presented in this appendix.

\subsection{Publications}

From the material and results of the thesis, seven independent research papers and one review paper have been produced (see Table.1.1). They have been either published or accepted for publication before completion of the thesis or will be submitted afterwards. I am the first author of the research papers with my supervisors Dr Paul Teal and Dr Mark McGuinness. Dr Grant Searchfield, of the University of Auckland, is a co-author for the paper from Chapter 5. I am a coauthor on the review paper with Dr Stephen J. Elliott and Dr Guangjian Ni, of Institute of Sound and Vibration, University of Southampton, United Kingdom. 
Table 1.1: Manuscripts published, accepted or in preparation for submission from the materials and the results of these research.

\begin{tabular}{|c|c|c|c|}
\hline Title & Chapter(s) & $\begin{array}{l}\text { Journal/ } \\
\text { Conference }\end{array}$ & Status \\
\hline $\begin{array}{l}\text { Modelling the generation of the } \\
\text { cochlear microphonic (Ayat and Teal, } \\
\text { 2013a) }\end{array}$ & $\begin{array}{lr}\text { based } & \text { on } \\
\text { Chapters } & 3 \\
\text { and } 4 & \end{array}$ & $\begin{array}{l}\text { EMBC2013 } \\
\text { IEEE Proceeding }\end{array}$ & $\begin{array}{l}\text { Published, } \\
\text { July } 2013\end{array}$ \\
\hline $\begin{array}{l}\text { Using Circuit Analogies for Analysis } \\
\text { of Cochlear Models (Ayat and Teal, } \\
\text { 2013b) }\end{array}$ & $\begin{array}{l}\text { Based on Ap- } \\
\text { pendix B }\end{array}$ & $\begin{array}{l}\text { Biomedical } \\
\text { Engineering } \\
\text { Letters (BMEL) }\end{array}$ & $\begin{array}{l}\text { Published, } \\
\text { Dec } 2013\end{array}$ \\
\hline $\begin{array}{l}\text { Cochlear microphonic broad tuning } \\
\text { curves (Ayat et al.) }\end{array}$ & $\begin{array}{l}\text { Based on } \\
\text { Chapter } 5\end{array}$ & $\begin{array}{l}\text { Mechanics } \\
\text { of Hearing } \\
\text { Proceeding }\end{array}$ & Accepted \\
\hline $\begin{array}{l}\text { Model based prediction of the ex- } \\
\text { istence of the spontaneous cochlear } \\
\text { microphonic (Ayat and Teal) }\end{array}$ & $\begin{array}{l}\text { Based on } \\
\text { Chapter } 6\end{array}$ & $\begin{array}{l}\text { Mechanics } \\
\text { of Hearing } \\
\text { Proceeding }\end{array}$ & Accepted \\
\hline $\begin{array}{l}\text { Modelling Cochlear Mechanics (a re- } \\
\text { view) (Ni et al., 2014) }\end{array}$ & $\begin{array}{lr}\text { Based } & \text { on } \\
\text { Chapters } & 3 \\
\text { and } 4 & \end{array}$ & $\begin{array}{l}\text { BioMed } \\
\text { Research } \\
\text { International } \\
\text { Journal }\end{array}$ & $\begin{array}{l}\text { Published, } \\
\text { July } 2014\end{array}$ \\
\hline $\begin{array}{l}\text { An Integrated Model for the Cochlear } \\
\text { Microphonic (Ayat et al., 2014) }\end{array}$ & $\begin{array}{l}\text { Based on } \\
\text { Chapters 3, } 4 \\
\text { and Appendix } \\
\text { C }\end{array}$ & $\begin{array}{l}\text { Biocybernetics } \\
\text { and Biomedical } \\
\text { Engineering } \\
\text { Journal }\end{array}$ & $\begin{array}{l}\text { Published, } \\
\text { July } 2014\end{array}$ \\
\hline Spontaneous Cochlear Microphonic & $\begin{array}{l}\text { Based on } \\
\text { Chapters } 6\end{array}$ & To be decided & In preparation \\
\hline $\begin{array}{l}\text { Broadness of the cochlear microphonic } \\
\text { tuning curves }\end{array}$ & $\begin{array}{l}\text { Based on } \\
\text { Chapter } 5\end{array}$ & To be decided & In preparation \\
\hline
\end{tabular}




\section{Chapter 2}

\section{Background}

The main goal of this thesis is to propose a model for the generation of the cochlear microphonic. In doing so, an introduction to the basic anatomy and physiology of the human ear will be needed. It is the function of this chapter to provide this introduction.

\subsection{Anatomy and Physiology of the Human Ear}

The human ear can be divided into three functional parts: the external (outer) ear, the middle ear and the inner ear. The pinna (auricle) and ear canal (external auditory meatus) comprise the external ear. The middle ear is made up of the eardrum (tympanic membrane), the tympanic cavity; the ossicular chain with its associated muscles, tendons, and ligaments; and the eustachian (auditory) tube. There are three small bones (ossicles) in the ossicular chain, the malleus, incus, and stapes. The inner ear is composed of the vestibule, the cochlea, and the vestibular apparatus (vestibular system) (Gelfand, 2010, Chapter 2); (Kandel et al., 2012, Chapter 30). Fig. 2.1 shows the different parts of the human ear.

Sound waves pass through the external auditory meatus, and vibrate the eardrum. The ossicular chain in the middle ear conducts the energy of vibrations of the 
eardrum to the oval window of the cochlea in the inner ear. The cochlea is a fluid-filled organ and sound reaches the ear by the way of air, therefore the middle ear can be considered as an impedance-matching transformer. Without this, the impedance mismatch between air and the cochlear fluids would cause much of the energy to reflect back rather than being transmitted through the cochlear fluids. The remarkable configuration of the middle ear efficiently transfers the sound energy to the cochlea over all audible frequencies (Fettiplace and Hackney, 2006). Without the middle ear only about $0.1 \%$ of the sound energy would be transmitted to the cochlea (Gelfand, 2010, Chapter 2).

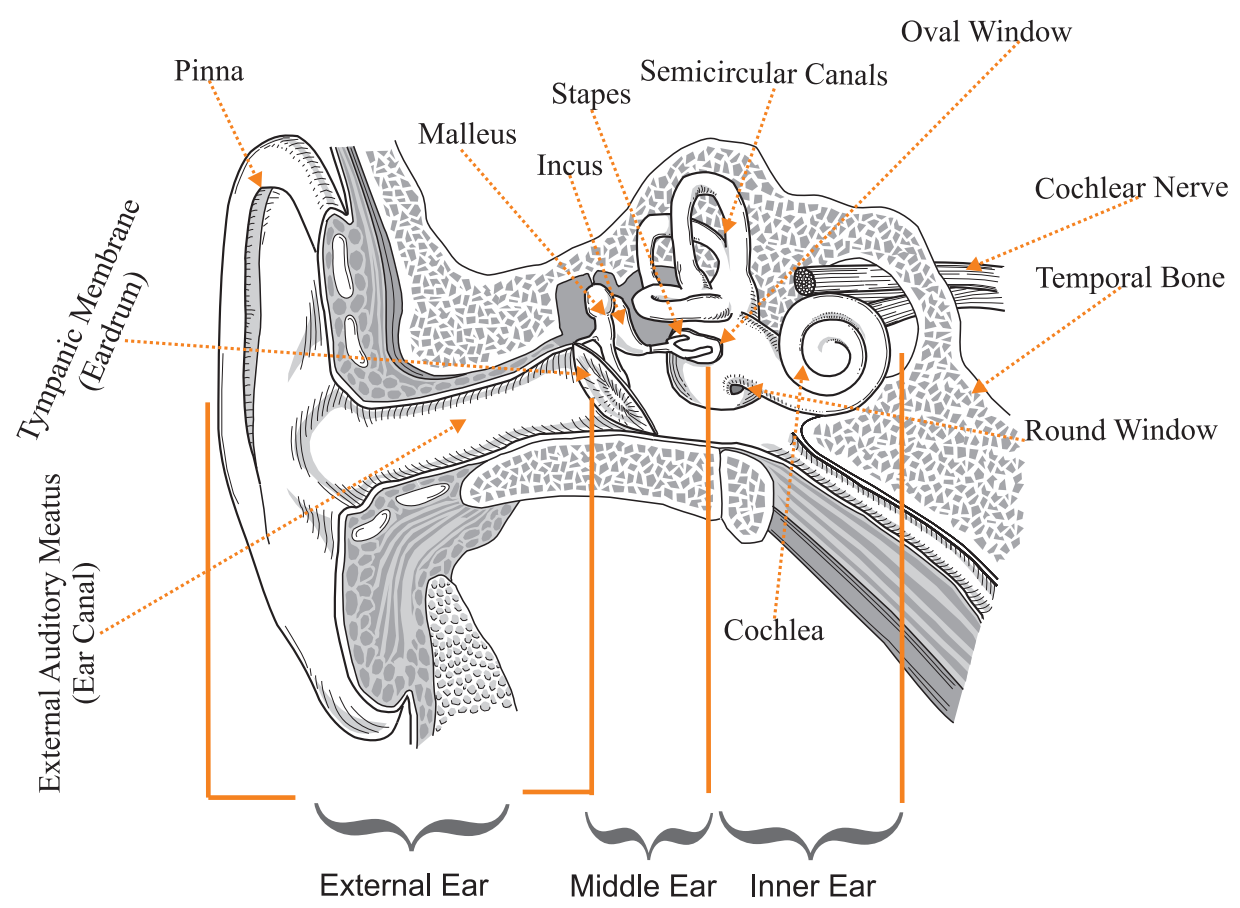

Figure 2.1: Cross-section of the human external, middle and inner ears. The middle ear is separated by the tympanic membrane from the external ear and by the oval window from the inner ear. Adapted from (Watts, 1993) with the author's permission. 


\subsubsection{The Human Cochlea}

The cochlea converts mechanical energy of pressure to action potential which can be interpreted by the nervous system.

\section{Anatomy}

The outer human cochlear wall is about 39 to $46 \mathrm{~mm}$ in length and makes a spiral with about 2.6 turns (Erixon et al., 2009). Fig. 2.2 shows an uncoiled cochlea. The BM and Reissner's membrane extend almost from base to apex. At the apex there is a small hole, called the helicotrema by which the scala vestibuli and scala tympani are joined. Pressure and ionic concentration in the scalae vestibuli and media equalise through the helicotrema (Watts, 1993). Reissner's membrane provides ionic isolation between the scala vestibuli and scala media (Watts, 1993). The scala vestibuli and the scala tympani are filled with the perilymph; a fluid with high sodium and low potassium content. The scala media between them is filled with endolymph; a fluid with low sodium and high potassium content. The difference in ionic concentration is controlled by a rich network of capillaries on the outer cochlear wall called the stria vascularis. The stria vascularis maintains an electrical potential difference named the endocochlear potential which can be considered as a battery for the hair cells in the organ of Corti (Wangemann, 2006).

The organ of Corti sits on the basilar membrane. The organ of Corti contains the hair cells. The Reticular Lamina $(R L)$ includes the tops of the hair cells (cuticular plates) (Furness and Hackney, 2008; Gelfand, 2010, Chapter 2). The organ of Corti is covered by a gelatinous and fibrous flap, called the tectorial membrane (Pickles, 2008, Chapter 2). A cross-section of the cochlea is depicted in Fig. 2.3. 


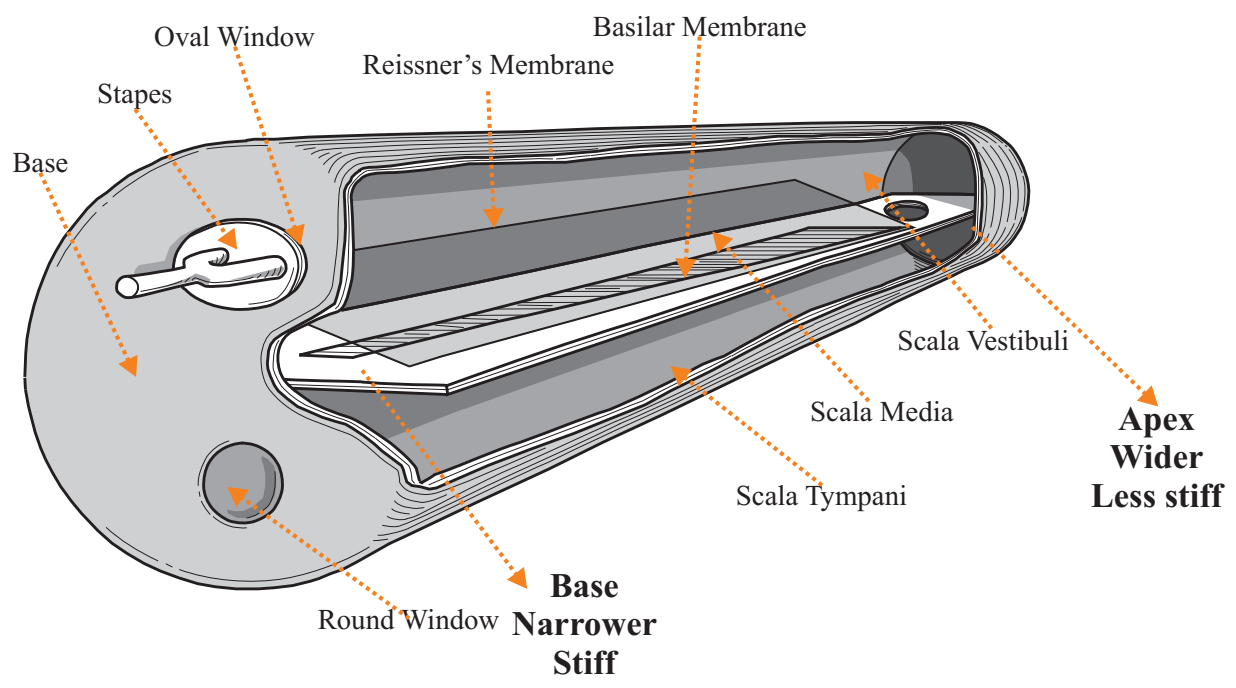

Figure 2.2: The representation of the uncoiled cochlea. The cochlea is made up of three fluidfilled chambers (scalae), the scala vestibuli, the scala media, and the scala tympani. Reissners membrane separates the scala vestibuli and the scala media and the basilar membrane separate the scala media and the scala tympani. The helicotrema connects the scala vestibuli and the scala tympani. From (Watts, 1993) with the author's permission.

\subsection{Hair cells}

The mammalian cochlea contains two classes of hair cells. There are approximately 3500 Inner Hair Cells (IHCs) and 11000 Outer Hair Cells (OHCs) in each human cochlea. The OHCs are grossly arranged in 3 rows in the human cochlea (see Fig, 2.4)(Ashmore, 2008). An array of the mechanosensory organelles which are called stereocilia ${ }^{1}$ protrudes on the apical surface of both classes of hair cells. This array is called the Hair Bundle (HB) (Martin, 2007). The tip of each stereocilium is connected to the adjacent taller stereocilium by a fine fibre which acts as a gating spring and is known as a tip link (Kandel et al., 2012, Chapter 31). Tip links have a fundamental role in the functioning of the hair cells. The role of tip links is discussed later in this chapter.

\footnotetext{
${ }^{1}$ It is because of the cilia that they are called hair cells.
} 


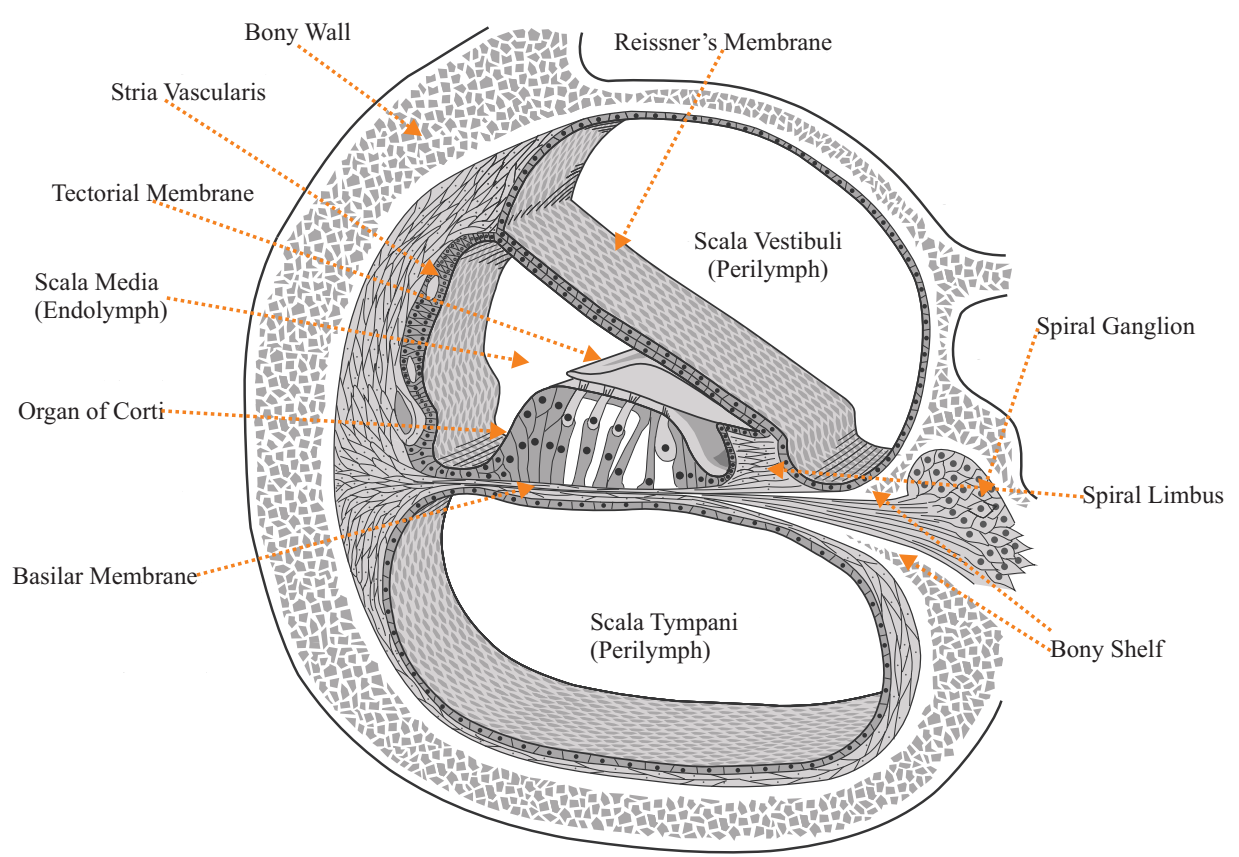

Figure 2.3: Cross-section of the cochlea. Reissner's membrane and the basilar membrane respectively separate the scala media from scala vestibuli above, and scala tympani below. The perilymph is the fluid in the scala vestibuli and scala tympani. The endolymph fills the scala media. From (Watts, 1993) with the author's permission.

The consummate arrangement of hair cells in the organ of Corti and their orientation enhance their function as sensory receptors. The IHCs convert the mechanical vibrations into electrochemical activities which are transferred to the brain for interpretation by auditory nerves. The OHCs convert these vibrations into electromechanical activities which modifies the BMs vibrations (Fettiplace and Hackney, 2006; Gelfand, 2010, Chapter 2).

These two types of hair cell are connected to the nervous system by way of the auditory (cochlear) branch of the eighth cranial nerve (Gelfand, 2010, Chapter 2). Ninety five percent of auditory afferent ${ }^{2}$ nerve fibres connect to the IHCs and are called type I auditory neurons, and five percent of them connect to the OHCs and are called type II auditory neurons (Gelfand, 2010, Chapter 2). Surprisingly, type

\footnotetext{
${ }^{2}$ Nerve fibres that carry signals to the brain.
} 


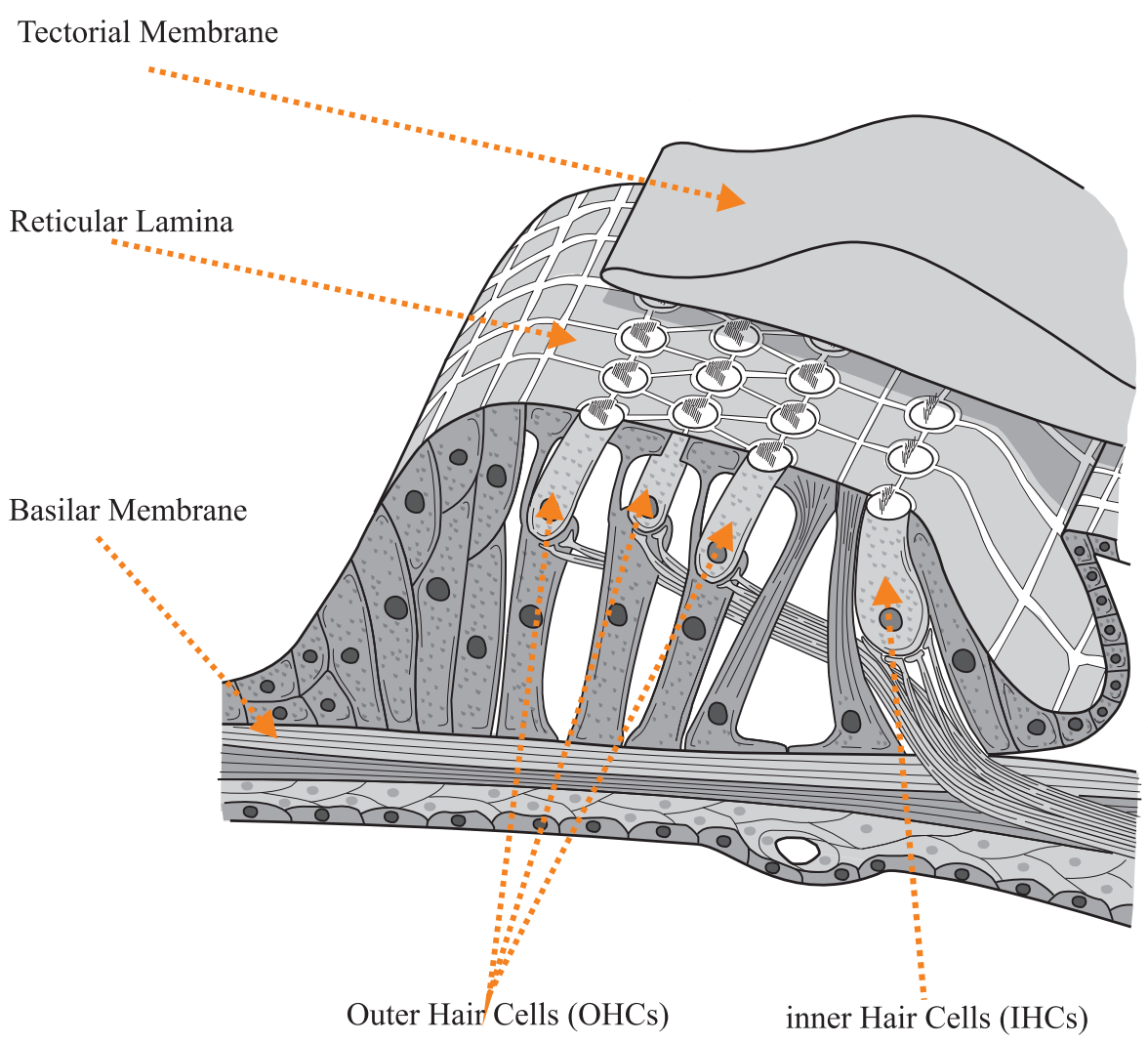

Figure 2.4: Cross-section of the organ of Corti. The organ of Corti is attached to the basilar membrane. It includes the hair cells, the tectorial membrane and the reticular lamina. From (Watts, 1993) with the author's permission.

II auditory neurons are found to be silent in response to acoustic stimulation, and their action is still unknown (Pickles, 2008). In contrast, the density of efferent ${ }^{3}$ nerve fibres is significantly greater for the OHCs than for the IHCs (Ashmore, 2008). The function of these efferent nerve is still disputed. They may control the electromechanical activities of the OHCs (Frolenkov, 2006; Moller, 2006, Chapter 3).

\footnotetext{
${ }^{3}$ Nerve fibres that carry signals from the brain.
} 


\section{Hearing Process and Cochlear Function}

The normal hearing process begins with capturing sound energy by the pinna. The human auditory system is capable of transducing a large dynamic range of sound pressure between $20 \mu \mathrm{Pa}$ to $20 \mathrm{~Pa}$. For convenience, in most acoustical measurements, the level of sound is expressed in decibels Sound Pressure Level (SPL), or dB SPL rather than Pascals, and calculated as:

$$
L_{\mathrm{dB} \mathrm{SPL}}=20 \log _{10}\left(\frac{P}{P_{\text {ref }}}\right)
$$

where $P$ is the measurement of the stimulus pressure level and $P_{\text {ref }}=20 \mu \mathrm{Pa}$. $P_{\text {ref }}$ is an approximation of the human hearing threshold at $4 \mathrm{kHz}$. Human ear is believed to be most sensitive at this frequency (Kandel et al., 2012, Chapter 30). By using this definition, the human ear is capable of capturing sound pressure in the range 0 to $120 \mathrm{~dB}$ SPL.

The cochlear function commences at the stapes which directs energy from the eardrum to the oval window. The movement of the oval window causes waves to travel through the fluid from the base toward the apex. The cochlea fluid is practically incompressible, so the wave is propagated by movement of the fluid and the BM (Kandel et al., 2012, Chapter 30). The round window moves in the opposite direction to make room for the incompressible fluid (Watts, 1993). Different stiffnesses along the cochlea cause natural or passive tuning of the cochlea; in other words, as the wave propagates down the cochlea, the stiffness decreases and the wave comes to a point; identified as the Best Place $(B P)$, (associated with the Characteristic Frequency $(C F)$ according to the placefrequency map see page 58) for a given input frequency, where the membrane will vibrate with maximum amplitude. Beyond that point the basilar membrane becomes less stiff and highly damped so the wave energy dissipates rapidly. This spatial tuning is further enhanced by active elements in the organ of Corti (Watts, 1993; Ku, 2008). The relationship between the tuning of a location and the distance from the base to apex is shown in Fig. 2.5.

The BM displacement deflects the stereocilia and activates the hair cells. This 


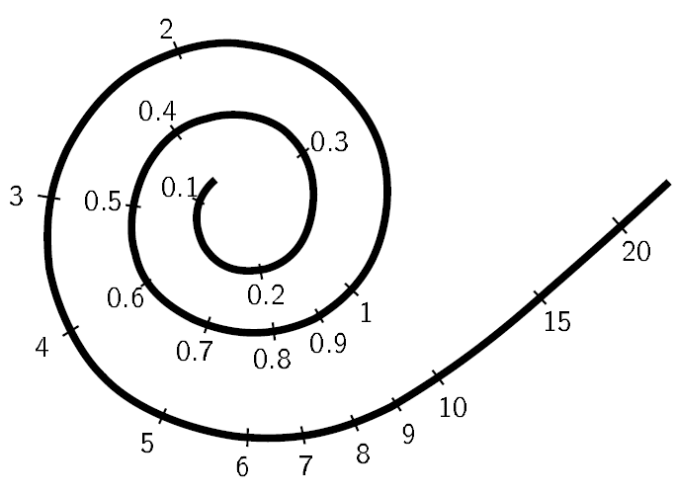

(a) Frequency map (in $\mathrm{KHz}$ ) along the cochlea

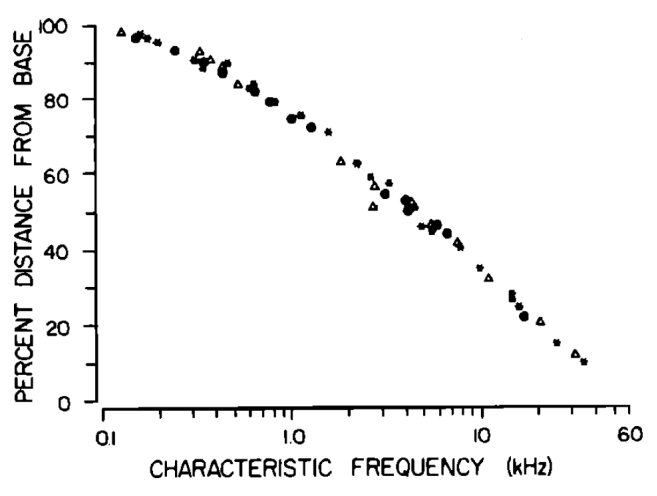

(b) The cochlear frequency map

Figure 2.5: a) Frequency map (in KHz) on the basilar membrane. Adapted from (Fletcher, 1938) b) relationship between frequency and distance in percent along the cochlear in a cat. From (Liberman, 1982) with permission of J. Acoust. Soc. Am.

procedure is depicted in Fig. 2.6. Hair cells convert mechanical to electrochemical activity in a process which is called Mechanoelectrical Transduction (MET) (Fettiplace and Hackney, 2006). Deflection of the stereocilia opens and closes pores known as MET channels (Fig. 2.7 shows how the tip links open and close the MET channels). Due to the voltage difference between the endolymph and the intracellular potential, the opening of the MET channels causes an inflow of ions $\left(\mathrm{K}^{+}\right)$, comprising a transduction current. Accordingly, the auditory neurons are stimulated by the IHCs (Fig. 2.8) and mechanical force is generated by the OHCs. The $\mathrm{K}^{+}$then is actively pumped back to the scala media via the spiral ligament and stria vascularis (Mistrík et al., 2009).

To sum up, the sound energy is converted into BM vibration, which leads to opening and closing of the MET channels that causes neurotransmitter release at the synapse on the auditory neurons in the IHCs and causes electromechanical activation of the OHCs. The longest stereocilium of the OHCs is attached to the tectorial membrane, but the stereocilia of the IHCs are not attached to tectorial membrane (Snow et al., 2009). Fig. 2.6 shows this difference. Experiments suggest an interpretation of this distinction: the responses of the OHCs are 


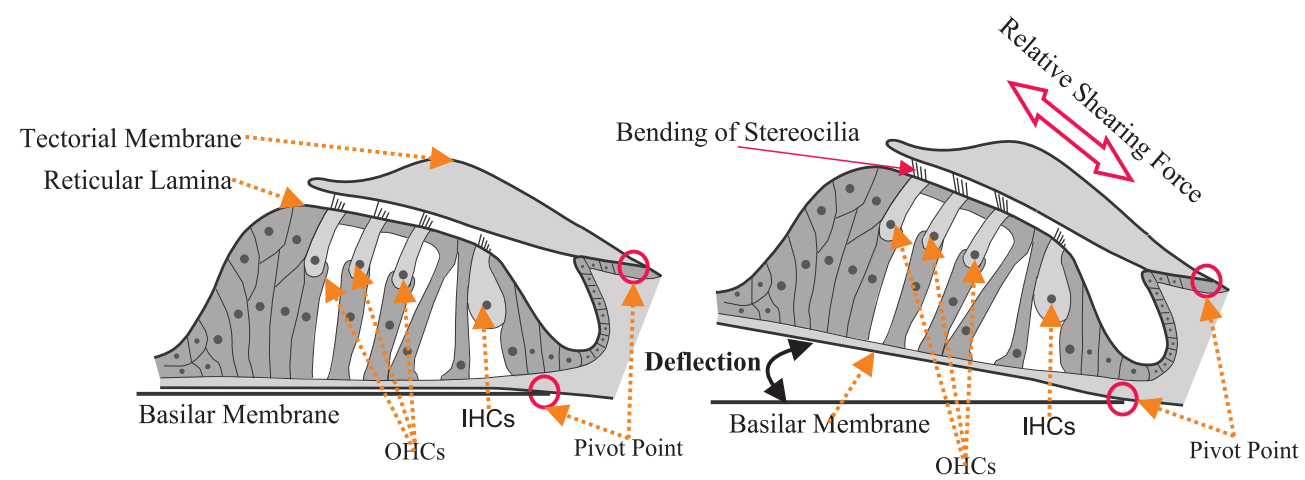

Figure 2.6: The shearing movement of the basilar and the tectorial membranes causes the stereocilia deflection by which the mechanical activity is converted into the electrochemical activity. OHCs are attached to the tectorial membrane whereas IHCs are not. Adapted from (Watts, 1993) with the author's permission.

proportional to the $\mathrm{BM}$ displacement which has attached cilia; whereas the responses of the IHCs are proportional to the velocity of basilar membrane which has freestanding cilia and hence is stimulated by fluid drag (Dallos et al., 1972); (Gelfand, 2010, Chapter 4). In other words, the MET currents of the OHCs depend on the BM displacements and the MET currents of the IHCs depend on the basilar membrane velocities.

\subsection{Active Processes and Cochlear Amplifier}

Early measurements of the neural tuning curve were significantly different from the mechanical tuning curve (Fig. 2.9). It was thought that there must be an intervening filtering process between the mechanical frequency selectivity along the cochlea (first filter) on the one side and between the basilar membrane and neural responses on the other side (second filter $)^{4}$. In the late 1960s, use of

\footnotetext{
${ }^{4}$ For an interesting history of the second filter see chapter two of Manley et al. (2008)
} 


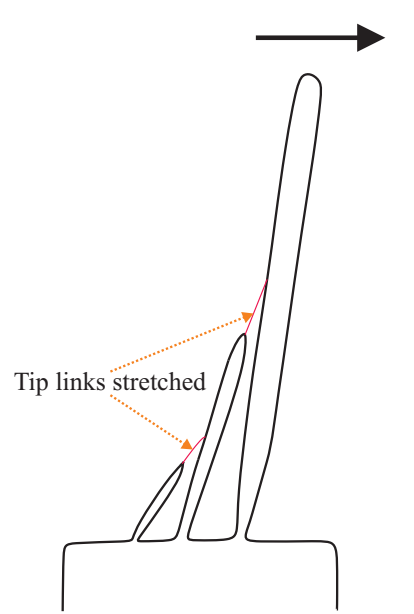

(a) tip links

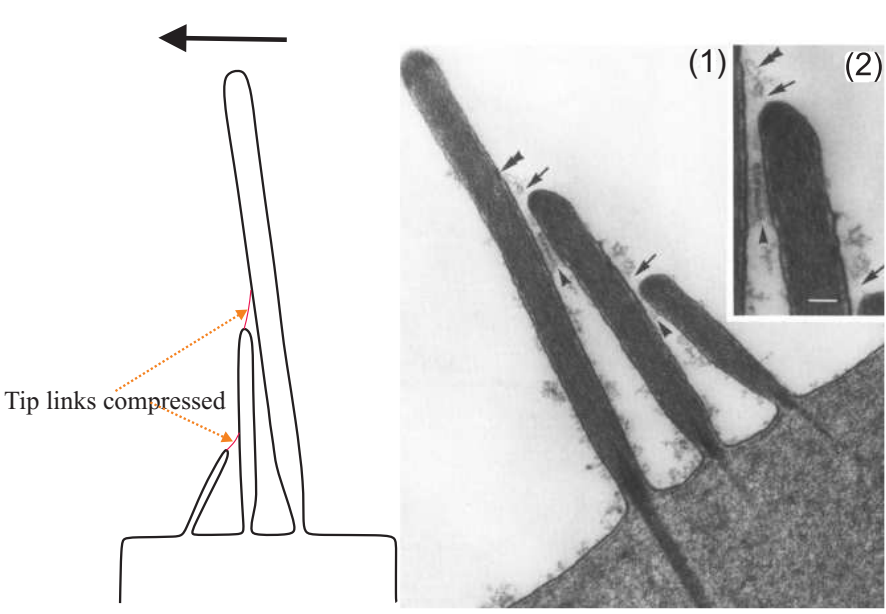

(b) Electron Microscopic view

Figure 2.7: a) Deflection of the stereocilia causes the tip links to open and close the mechanoelectrical transduction (MET) channels. Source Pickles et al. (1984) with permission b) (1) Electron Microscopic view of tip links (2) Closer view with more details. Source (Osborne et al., 1988) with permission.

the Mössbauer technique ${ }^{5}$ made measurement from undamaged (alive) cochleae possible. In the late 1970s, improvements in measuring techniques and different experiments demonstrated that the mechanical tuning curves are as sharp as the neural tuning curves. In addition (Kemp, 1978a) showed that the cochlea does not only absorb sound, it can also emit sound. Kemp's finding was a turning point in hearing research history; his studies substantiated that not only is the cochlea mechanically active, but also that it is mechanically nonlinear (Manley et al., 2008).

Kemp found that sound energy is emitted back by the cochlea some tens of milliseconds after impulsive acoustic excitation. This behaviour of the cochlea was originally referred to as the Kemp echo and later called the evoked or

\footnotetext{
${ }^{5}$ In this technique a small radioactive source is placed on the basilar membrane and the variation in the frequency of the emitted radiation is detected (Manley et al., 2008); (Gelfand, 2010, Chapter 4)
} 


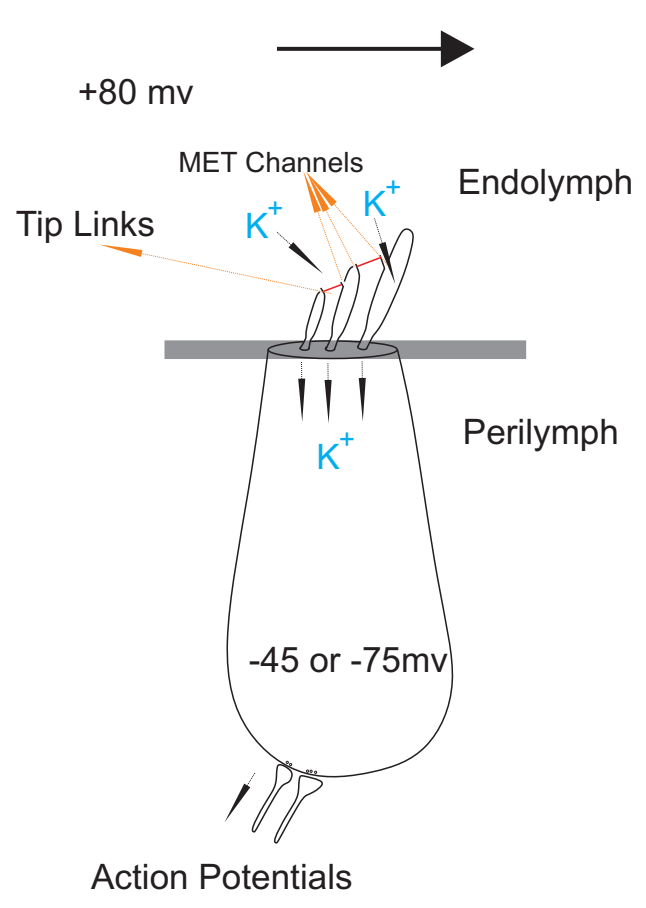

Figure 2.8: The operation of hair cells; deflection of the stereocilia opens and closes the MET channels which lead to ion flow, driven by the voltage difference between the endolymph and the intracellular potential.

stimulated otoacoustic emission ${ }^{6}$ (Gelfand, 2010, Chapter 4). Using Otoacoustic Emissions (OAEs) provided a powerful investigative technique for audiologists to examine the cochlea (for extensive reviews see Probst et al. (1991) and Manley et al. (2008)). The simplicity of this method has discouraged the development of other methods such as using the cochlear microphonic as a method for scrutinising the cochlea.

Spontaneous Otacoustic Emissions (SOAEs) are low-level vibrations which are spontaneously produced by the cochlea and can be measured in the external ear in the absence of stimulus (Probst et al., 1991). Their presence is an indicator of normal hearing sensitivity near the frequency of an SOAE (Bright, 2002). Selfoscillation of the OHCs is believed to be the source of these kinds of emissions

\footnotetext{
${ }^{6}$ Oto-: Prefix meaning ear, as in otoscope (a medical device for looking inside the ear).
} 


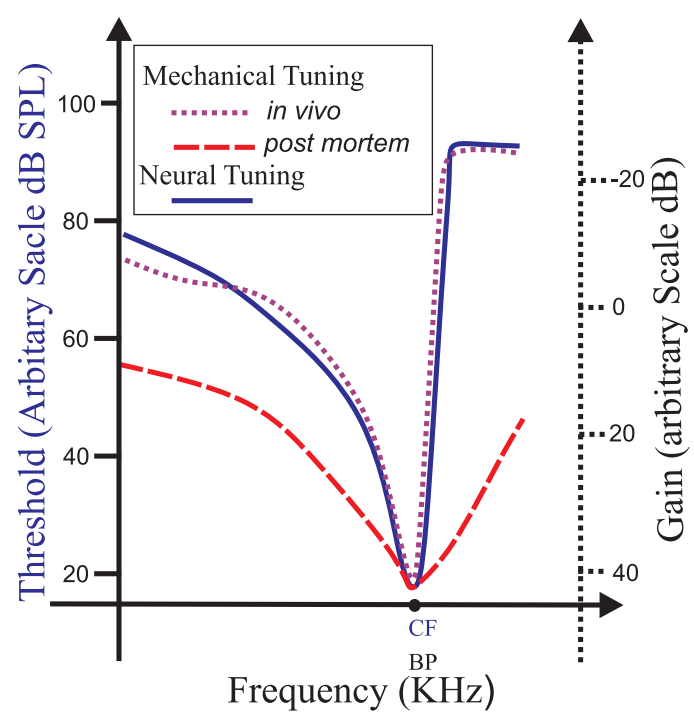

Figure 2.9: A comparison between neural and mechanical tuning curves. The Ordinate on the left side illustrates the threshold level of different sound pressure levels (SPL) for the neural tuning curve and, the ordinate on the right side shows the gain for the mechanical tuning curve. The indicator on the abscissa shows the characteristic frequency (CF) for the neural tuning curve and the best place (BP) for the mechanical tuning curve. The feasibility of in vivo measurements of the basilar membrane displacement demonstrated that the basilar membrane displacement is just as sharply tuned as the auditory nerve response, and the need for a second filter concept vanished. Prior to that, researchers thought that there was no important difference between in vivo and post mortem measurements and another process should intervene to convert the broadly tuned mechanical curve to the sharply tuned neural curve.

(Moller, 2006, Chapter 3). Some evidence in mammals suggest that SOAEs stem from coherent multiple reflection of travelling waves which are maintained and stabilised by the nonlinear cochlear amplifier (Shera, 2003; Guinan Jr et al., 2012). This hypothesis has been challenged by both clinical data and modelling measurements (Braun, 2013).

Even though the SOAE is not routinely used for clinical applications, it is a valuable tool for hearing investigation (Hall, 2000). SOAEs are a clear indication of the active process in the cochlea which can be used to better understand the cochlear mechanism (Vilfan and Duke, 2008). 


\subsection{What is the Source of the Active Process?}

As mentioned earlier, innervations of the cochlea suggest that the IHCs rather than the OHCs are involved in perception or converting vibration to neuron impulses. The OHCs are mostly engaged in the active process in the cochlea. The active process relies on $\mathrm{OHC}$ electromotility. The $\mathrm{OHC}$ provides the forces that are added to the fluid forces in the cochlea and thus acts as an amplifier. This process can be characterised as a positive feedback loop within the cochlea that amplifies the travelling wave (Oghalai, 2004). The active process allows the cochlea to detect input stimulus with a large dynamic range. The $\mathrm{OHC}$ in the mammalian cochlea is thought to use both somatic electromotility and hair bundle electromotility to provide mechanical active amplification (Nam and Fettiplace, 2010; Maoiléidigh and Hudspeth, 2013).

\subsubsection{Hair Bundle Motility}

Hair bundle motility is considered by some researchers to have an effective amplification property in mammalian hair cells organs (Peng and Ricci, 2011). The HB uses the motor protein myosin to cause a force encountering the stimuli (Fettiplace and Hackney, 2006). The hair bundle force is linked to the displacement of the hair bundle and the probability of the opening of the MET channels (Fettiplace, 2006). The hair bundle electromotility mechanism was put forward to explain amplification at high frequencies for which the membrane time constant was thought to restrict OHC amplification (Kennedy et al., 2005).

More recent measurement showed that membrane time constant does not limit OHC amplification. The membrane time constant is approximately an order of magnitude smaller than what was previously reported (Johnson et al., 2011; Nam and Fettiplace, 2012) (refer to Section C.2.2 and (Ashmore, 2011)). Experimental data indicates that somatic electromotility of the $\mathrm{OHC}$ is much larger than hair bundle motility, and is therefore the primary amplification mechanism of mammalian OHC (Liberman et al., 2002; Lagarde et al., 2008). The force 
intensity and purpose of the hair bundle electromotility mechanism in the mammalian cochlea are disputed and still require more investigation (Nam and Fettiplace, 2010).

\subsubsection{Somatic Motility}

Change to the length of the $\mathrm{OHC}$ is an active mechanism which makes the mammalian cochlea remarkably sensitive and precisely frequency sensitive. The OHC length depends on the hair cell membrane potential which in turn depends on the current flowing through the MET channel. Both of these effects are nonlinear. The MET channel current can be described as a Boltzmann function of hair bundle displacement (Ashmore, 2008) and this is the primary source of nonlinearity. The somatic motility of the $\mathrm{OHCs}$ relies on a unique motor protein of the $\mathrm{OHC}$ which is called prestin (Gelfand, 2010, Chapter 4).

By using some simplifications, it can be shown that the ratio of OHC length change to the membrane charge $(\mathrm{Q})$ movement is approximately constant and the OHC can be modelled as a piezoelectric material (Mountain and Hubbard, 1994). A capacitance can be calculated as the first derivative of $\mathrm{Q}$ with respect to cell membrane voltage V (Liu and Neely, 2009). Although this effect is also nonlinear, the relationship is usually considered to be approximately linear. These relations couple electrical property of the $\mathrm{OHC}$ soma with the $\mathrm{OHC}$ mechanics and represent the $\mathrm{OHC}$ somatic motility (Nam and Fettiplace, 2012).

\subsection{Electrical Activities Inside the Cochlea}

In the most general term, hair cells detect vibrations, reduce their dynamic range and encode them to a form that the nervous system can interpret. These processes rely on electrical activities inside the cochlea. When sounds (stimuli) reach the inner ear, the structures of the cochlea, as we have seen so far, convert them to electrical signals. These electrical signals which are called the gross evoked 
potential can be measured by placing electrodes near the cochlea (Pickles, 2008).

These signals are made of three different components (Fig. 2.10). Firstly, the Cochlear Microphonic (CM) is an AC signal nearly identical to the acoustic AC signal and is referred to as the steady-state part of the response. Secondly, the series of deflections of the beginning and sometimes at the end of a stimulus, called the Compound Action Potential (CAP) are a summation activity of auditory nerve fibres. Finally, there is a baseline shift in the recording of the gross evoked potential known as the Summating Potential (SP), which depends on the DC component of the hair cell current (Cheatham et al., 2011; Pickles, 2008).
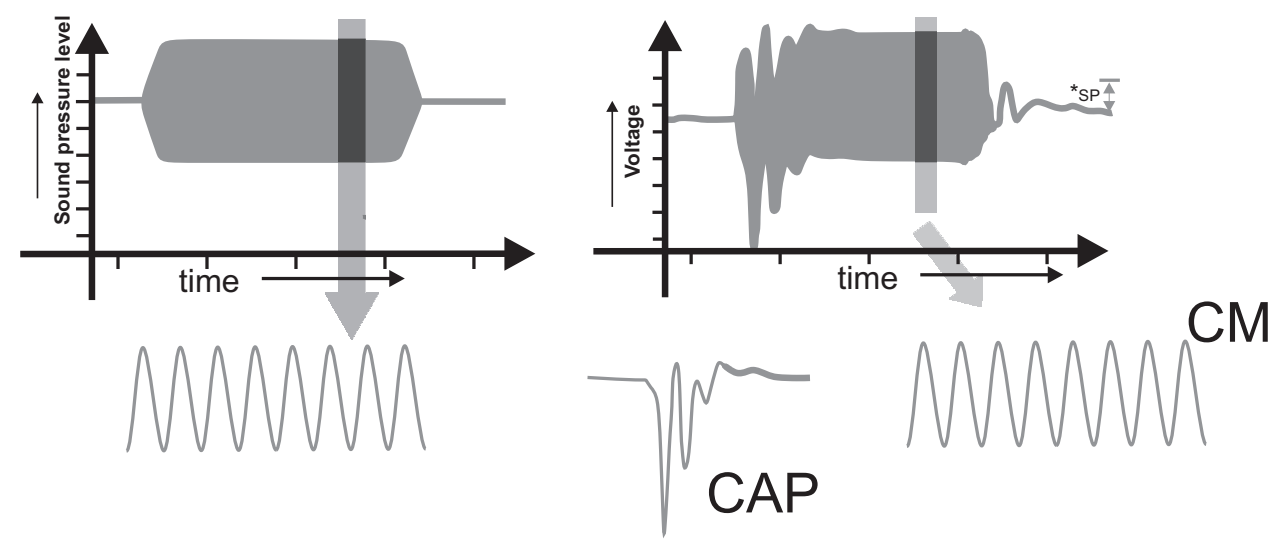

Figure 2.10: a) Tone-burst stimuli presented the ear. b) Response to the tone-burst as recorded from the round window. CM is the steady-state part of the response, while CAP is the transient portion of the response. From (Cheatham et al., 2011) with permission of JARO.

(*SP is not shown on original figure, Here, it has been added to show the three different parts of gross evoked potential.)

\subsubsection{Cochlear Microphonic}

Recall from Section 2.2 that deformation of the BM causes deflection of the stereocilia which open and close MET channels. This alternation causes an electrical current through the biological resistances and capacitances in the organ of Corti which is the source of the CM. The relationship between deflection and 
current magnitudes is non-linear.

The CM can be recorded by placing electrodes on or close to the round window membrane, or the surface of the cochlea or with glass micropipette electrodes inserted in the scala media near the site of generation (Russell, 2008; Liberman et al., 2002; Honrubia and Ward, 1968). The majority of human clinical and laboratory applications of CM recording are based on recordings invasively from the niche of the round window through transtympanic electrocochleography (He et al., 2012b). Improvement in measuring techniques has also permitted the reliable non-invasive recording of these signals (Masood et al., 2012; Poch-Broto et al., 2009).

\subsubsection{A Concise History of the Cochlear Microphonic}

In several papers in 1930, Wever and Bray explained their extraordinary discoveries. In their experiments they tried to pick up auditory nerve signals of a cat by placing electrodes near those nerves. They observed that the cat's ear converted sounds to electrical signal with great fidelity which could be heard over a speaker (Wever and Bray, 1930a,b). They thought that they had been checking the auditory nerve alone, but, very soon after that Adrian (1931) criticised their interpretation. His main reason was that the electrodes were too distant to observe the action potential of nerves. Other researchers found that this response is stronger near the round window than at the nerve and it can be still recorded even if the nerve was destroyed (Gelfand, 2010, Chapter 4). Adrian (1931), in conclusion, stated that this effect was related to some kind of microphonic action. Since then the term Cochlear Microphonic (CM) has been used to describe this signal. By doing several interesting experiments, Békésy (1951) tried to answer this question: what is the energy source for the CM? He concluded that the electrical energy of the $\mathrm{CM}$ is provided not only by the mechanical energy of stimuli in a healthy ear, but also by dc potentials along the cochlea. Tasaki and Fernandez (1952); Tasaki et al. $(1952,1954)$ used microelectrodes to explore the cochlear potentials. Their results indicated that the source of the $\mathrm{CM}$ is at the hair-bearing end of the hair 
cells. Then researchers proved that the $\mathrm{CM}$ is produced by both inner and outer hair cells (Withnell, 2001). However, there are some results showing that the outer hair cell contribution is greater (Dallos, 1983; Gelfand, 2010, Chapter 4).

\subsubsection{Applications of the Cochlear Microphonic}

The clinical use of the $\mathrm{CM}$, and in particular its application to assessment of cochlear performance has been restricted because of difficulty in recording the CM and uncertainty in its interpretation (Santarelli et al., 2006). There are however several applications of the CM that have been established in laboratory and clinical research including checking the biological effects of infrasound on the human auditory system (Salt and Kaltenbach, 2011), diagnosing Ménière's disease (Harris and Salt, 2008), assessing MET current of outer hair cells (Patuzzi and Moleirinho, 1998; Patuzzi and O'Beirne, 1999), diagnosis of auditory neuropathy spectrum disorder (Starr et al., 2008) and using the CM as an objective audiological diagnosis test (Poch-Broto et al., 2009).

\section{Responses of the Cochlea to Infrasound}

Exposure to infrasound ${ }^{7}$ generated by wind turbines at the levels that may not be heard can still influence cochlear function. Experimental data demonstrates that infrasonic frequencies with low level amplitude which cannot be perceived by the human ear can still produce measurable $\mathrm{CM}$. These measurements indicate that the $\mathrm{OHCs}$ are functioning at infrasonic frequencies and hence the functionalities of the cochlea can be influenced by inaudible infrasound (Salt and Kaltenbach, 2011; Salt and Hullar, 2010; Chen and Narins, 2012). Prior to this finding infrasound from wind turbines was previously assumed to have no effect on the auditory system (Leventhall, 2006; Salt et al., 2013).

\footnotetext{
${ }^{7} \mathrm{~A}$ sound with frequency below $20 \mathrm{~Hz}$ is sometimes considered inaudible to the human ear and is hence called infrasound. This can be a misleading term however, since a sound at frequencies lower then $20 \mathrm{~Hz}$ with large magnitude can be audible to the human ear (Leventhall, 2007).
} 


\section{Diagnosing Ménière's Disease}

Ménière's disease is a chronic illness of the inner ear which causes fluctuating and progressive hearing loss (Harris and Salt, 2008). Two different manifestations of Ménière's disease can be distinguished by the CM. Some Ménière's disease patients exhibit large $\mathrm{CM}$ amplitudes. In these patients the cochlear mechanics is impaired but the hair cells are intact. In other patients hair cells damage has occurred and the CM amplitude is low. Hearing in patients with damaged cochlear mechanics may be recoverable if the underlying endolymphatic hydrops ${ }^{8}$ can be corrected (Norman et al., 1997).

\section{MET Current of OHCs}

The $\mathrm{CM}$ allows in vivo monitoring of changes in the OHC MET which are caused by cochlear disturbances such as asphyxia, acoustic over-stimulation, neural blockade,and drug perfusion (Patuzzi and Moleirinho, 1998; Patuzzi and O’Beirne, 1999).

\section{Diagnosis of Auditory Neuropathy Spectrum Disorder}

Auditory Neuropathy Spectrum Disorder (ANSD) (previously referred to as Auditory Neuropathy (AN) or Neuropathy / Auditory Dys-synchrony (AN/AD)) is a hearing disorder in which the outer hair cells are present and functional while the auditory nerve does not transfer the information correctly (Starr et al., 2008). The $\mathrm{CM}$ can play an important role in the diagnosis of ANSD patients. Experimental data show that amplitude level of the CM in patients with Distortion product OAEs (DPOAEs) ${ }^{9}$ can be used to distinguish between types of ANSD (Berlin et al.,

\footnotetext{
${ }^{8}$ A swelling of the endolymphatic space (membrane-bound endolymphatic compartment) of the inner ear which alters cochlear mechanics in patients with Ménière's disease (Harris and Salt, 2008).

${ }^{9}$ When two pure tone stimuli are presented into the ear, evoked responses with new frequencies can be measured from the cochlea which are called DPOAEs (Manley and van Dijk, 2007).
} 
2003; Shi et al., 2012).

\section{The CM as an Objective Audiological Diagnosis Test}

Experiments indicate that for cochlear implants to be effective, auditory experiences and neural activities are essential in early childhood (Chertoff et al., 2012). Otherwise the nerve fibres and auditory structure degenerate which impedes any future rehabilitation by cochlear implants. Therefore, it is very important to detect hearing problems in new born babies and children (Leake et al., 2006). Performing subjective audiometry test is very difficult and sometimes unreliable in infants and children and they should examined by objective audiometry (Tyberghein and Forrez, 1971). The CM can complement other objective tests like tympanometry and otoacoustic emissions or be an alternative to them (PochBroto et al., 2009). The CM may also be able to be used to determine the location of missing OHC along the cochlea (Chertoff et al., 2012).

\subsection{Summary}

A sound (stimulus) vibrates the eardrum and vibrations reach the inner ear (cochlea) through the ossicular chain of the middle ear. The vibration of the ossicles and consequently the oval window produces transversal waves travelling along the BM from the base towards the apex. As the wave travels inside the cochlea, the stiffness of the BM decreases and the membrane amplitude reaches its maximum at a point which is called the best place for each input frequency. The transversal movement of the BM stretches and relaxes the tip links that connect adjacent stereocilia together. The HB actively resists these changes and provides forces encountering the stimuli which are referred to as hair bundle motility. In addition, the changing tension in the tip links cause opening and closing of the MET channels which produces a transduction current through these channels. The transduction current causes depolarisation and hyperpolarisation of 
the outer hair cell membrane. This process results in contraction and extension of the OHCs which is referred to as somatic motility. Both the hair bundle motility and somatic motility provide forces that are added to the fluid forces in the cochlea, and thus act as an amplifier. This process is called the active mechanism of the cochlea.

OAEs are sounds which are produced in response to stimuli by the cochlea or are spontaneously generated without stimuli by the cochlea. These emissions are clear evidence of the active mechanism in the cochlea.

The transduction currents are distributed through the biological resistances and capacitances in the organ of Corti. The resulting potential is called the CM. The CM can be recorded by placing electrodes on or close to the round window membrane, or with electrodes that are placed in the outer ear canal or internally with glass micropipette electrodes inserted in the scala media.

Some clinical and research application of the CM include assessing the biological effects of infrasound on the human auditory system, diagnosing Ménière's disease, assessing the MET current of OHCs, diagnosis of auditory neuropathy spectrum disorder and using the $\mathrm{CM}$ as an objective audiological diagnostic test. 


\section{Chapter 3}

\section{Modelling}

\subsection{Introduction}

The human cochlea is enclosed in the hardest bone structure of the human body, which makes it inaccessible (Duifhuis, 2012, Chapter 1). Even with very sophisticated and accurate measuring techniques which are nowadays available, in vivo measurements of the cochlea are very difficult and sometimes impossible. To overcome this difficulty and maximise the utility of available data, models can be used. In addition models can reveal any parts of the hearing procedure that have been miscalculated, misspecified or misunderstood. For example, many of the initial measurements of the cochlea were done by von Békésy (Von Békésy and Wever, 1960). He had to use high level stimulus sound (up to $150 \mathrm{~dB}$ SPL; higher than the $120 \mathrm{~dB}$ SPL pain threshold) to observe the BM vibration in cadavers. He then approximated the lower level excitation profile by using the high level excitation profile. It was predictable that at those high stimulus levels the cochlea ceased functioning properly or was damaged permanently. By this approximation the low level BM displacement should be less than $1 \mathrm{pm}\left(1 \times 10^{-12} \mathrm{~m}\right)$ which was much lower than the BM displacement at the hearing threshold (in the order of $1 \mathrm{~nm}\left(1 \times 10^{-9} \mathrm{~m}\right)$ ) (Robles and Ruggero, 2001; Duifhuis, 2012, Chapter 2). 
His results and those of other researchers indicated that some other process or mechanism should be involved (Rhode, 1984). Researchers then began to search for the missing piece of the puzzle. Later by using the Mössbauer technique, Rhode (1971) showed that the BM vibration in a living cochlea exhibits nonlinear behaviour. In the ensuing years, different measurements indicated that an active process which is now called the cochlear amplifier is involved ${ }^{1}$.

We now know that if von Békésy had done his experiments on live and healthy cochleae, the BM displacement of low level stimulus would have been much larger (Guinan Jr et al., 2012). In addition, we now know that the $\mathrm{OHC}$ is the key element of the cochlear amplifier. These individual discoveries about the process of hearing can be integrated by using modelling techniques. Reasonable anticipations which can be inferred from the cochlear models can also lead to appropriate experiments and consequently may unveil unknown aspects of this remarkable organ.

In general terms, an acceptable model of the cochlea should reproduce the relevant observations. In addition, the model should make predictions about that organ which can be validated or invalidated by experiments or future observations.

All modelling approaches try to observe the cochlea from particular pertinent angle. For example in the modelling of OAEs, electrical properties of the $\mathrm{OHC}$ are not normally considered. In this work electrical activities inside the cochlea are very important.

An overview of models of cochlear mechanics and models of electrical coupling are presented in Sections 3.2 and 3.3. The remainder of the chapter is dedicated to an in-depth representation of the proposed model.

\footnotetext{
${ }^{1}$ In 1948, Thomas Gold reported that "the assumption of a 'passive' cochlea, where elements are brought into mechanical oscillation solely by means of the incident sound, is not tenable." and suggested a feedback stage. He also suggested some experiments to test this hypothesis (Gold, 1948). However his insight was ignored for more than two decades.
} 


\subsection{Models of Cochlear Mechanics}

\subsubsection{Mechanical properties of the cochlea}

As was presented in Chapter 2, the cochlea is a coiled bony labyrinth which contains three fluid-filled compartments. The upper compartment (scala vestibuli) is separated from the middle one (scala media) by a membrane called Reissner's membrane. The middle compartment is separated from the lower one by the BM (Patuzzi, 1996). The BM consists of transverse beamlike fibres that make it stiffer at the base and less stiff at the apex (Iurato, 1962). Reissner's membrane is very thin (two cells thick) and flexible, so the mechanical influence of it can be neglected (Patuzzi, 1996);(Gelfand, 2010, Chapter 4). The curvature of the cochlear geometry is believed to not have a significant role on the cochlea mechanics (Steele and Zais, 1985; Kohllöffel, 1990; Ni et al., 2011). It seems that the primary purpose of the coiling is to save space. However, some modelling results indicate that coiling intensifies the apical shear gain which efficiently improves the hair bundles deflections at the apex (Cai et al., 2005). In modelling, the uncoiled cochlear shape has usually been used.

Cochlear models usually include cochlear macromechanics and cochlear micromechanics. Cochlear macromechanics represents mechanical and dynamic behaviour of fluid inside the cochlea. Cochlear micromechanics represents the mechanical and dynamic behaviour of a radial slice of the organ of Corti and basilar membrane. Fig. 3.1 illustrates cochlear macromechanics and micromechanics.

\subsubsection{Cochlear Macromechanics}

\section{Hydrodynamics}

Newton's second law states that the overall force on a particle is equal to the rate of change of its linear momentum. This law can be used to derive the Navier-Stokes 


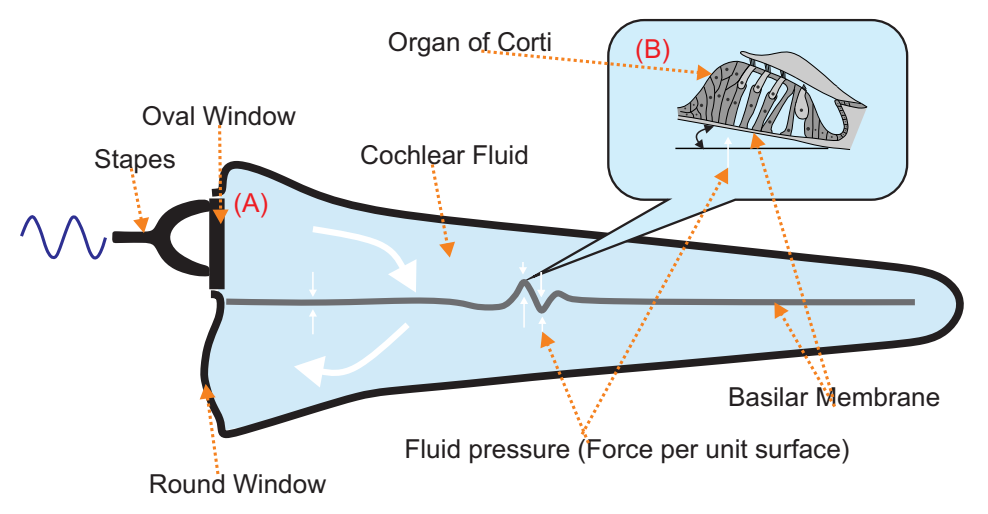

Figure 3.1: (A) shows macromechanics, which is the modelling of fluid pressure, force and velocity. (B) shows micromechanics which is the modelling of dynamic behaviour of a radial slice of the cochlea at the microscopic level. Recreated from Nobili et al. (1998) with permission

equation which governs the motion of an incompressible Newtonian fluid:

$$
\rho\left(\frac{\partial u}{\partial t}+(\nabla \cdot u) u\right)=-\nabla P+\mu \nabla^{2} u+\rho g
$$

where $\mu$ is dynamic viscosity, vector $P$ is pressure, $g$ is the acceleration of gravity vector, $u$ is the velocity and $\rho$ is the density of the fluid ${ }^{2}$.

\footnotetext{
${ }^{2}$ If vector $P$ and $u$ are represented in cartesian co-ordinates, then the gradient operator $\nabla$ can be represented as:

$$
\nabla=i \frac{\partial}{\partial x}+j \frac{\partial}{\partial y}+k \frac{\partial}{\partial z}
$$

where $i, j, k$ are the unit vectors in the respective directions.
} 
The principle of continuity is represented by ${ }^{3}$ :

$$
\nabla \cdot u=0
$$

The fluid inside the cochlea is typically assumed to be incompressible and inviscid $^{4}$ and gravity effects are ignored. Accordingly, the Navier-Stokes equation is usually simplified to (Pozrikidis, 2008; Keener and Sneyd, 2009a, Chapter 20):

$$
\rho \frac{\partial u}{\partial t}+\nabla P=0
$$

By taking the divergence of both sides of (3.2), the following equation can be obtained:

$$
\nabla^{2} P=0
$$

Equations (3.2), (3.3) and (3.1) are valid for three dimensions, but they are usually simplified to one or two dimensions (Neely, 1977).

\subsubsection{Cochlear Micromechanics}

The organ of Corti can be modelled as a series of radial sections arranged from the base to the apex. The resonant frequency of each radial section is determined

\footnotetext{
${ }^{3}$ The rate of change of the mass residing in region $\mathrm{V}$ inside an oriented surface $\mathrm{S}$ is equal to the mass flow rate inward through the surface or to the negative of the mass flow rate outward through the surface. Thus (using the divergence theorem or Gauss' theorem):

$$
\frac{d}{d t} \iiint_{V} \rho d v=-\iiint_{V} \nabla \cdot(\rho u) d v
$$

Since $\mathrm{V}$ is fixed in space, the order of integration and time differentiation on the left-hand side of this equation can be interchanged and the two sides of it can be combined to form the following equation (Pozrikidis, 2009, Chapter 2):

$$
\iiint_{V}\left(\frac{\partial \rho}{\partial t}+\nabla \cdot(\rho u)\right) d v=0
$$

Since $\rho$ is constant and V is arbitrary, equation (3.1) is straightforward.

${ }^{4}$ In some models, the fluid inside the cochlea modelled as a compressible, viscous fluid. For a review of these assumptions see Cheng et al. (2008); Ceresa et al. (2013).
} 
by the mass, stiffness and damping of the basilar membrane at that radial section. The basilar membrane has lower mass and higher stiffness at the base and higher mass and lower stiffness at the apex. Therefore the basilar membrane vibrates with maximum amplitude in response to high frequency stimuli at the base and in response to low frequency stimuli at the apex. However this passive property cannot explain the remarkable sensitivity and frequency selectivity of the cochlea (Oghalai, 2004). In vivo measurements of the basilar membrane vibration demonstrate both sharp tuning and sensitivity. Different experiments have shown that the mechanical effects of the OHCs are the reason for this sharp tuning and sensitivity. The OHCs sense vibrations in the cochlea and feed back mechanical force to the basilar membrane. This active process remarkably improves both cochlear sensitivity and frequency selectivity (Oghalai, 2004; Holley, 1996) (also refer to Section 2.4).

Different assumptions about the structure of the organ of Corti and the active force exerted by the OHCs can lead to a wide variety of configurations of cochlear micromechanics which result in different models (Patuzzi, 1996). Neely and Kim (1986)'s model is one of the famous ones. Fig. 3.2 shows this model. This model has been meticulously discussed in Elliott et al. (2007) and Ku (2008).

\subsection{Models Of Electrical Coupling}

\subsubsection{Electrical Properties of the Cochlea}

Recall from 2.1.1 that the three compartments of the cochlea are filled with two fluids called endolymph and perilymph. The endolymph, which fills the scala media has a unique ion content which makes it more electrically positive than both the perilymph and intracellular potentials. These differences in potential levels produce standing flows of ions (i.e., electrical currents) through various structures of the cochlea which maintain steady state potentials and currents in the cochlea. It is noteworthy to mention that the unique ion content of the endolymph is 


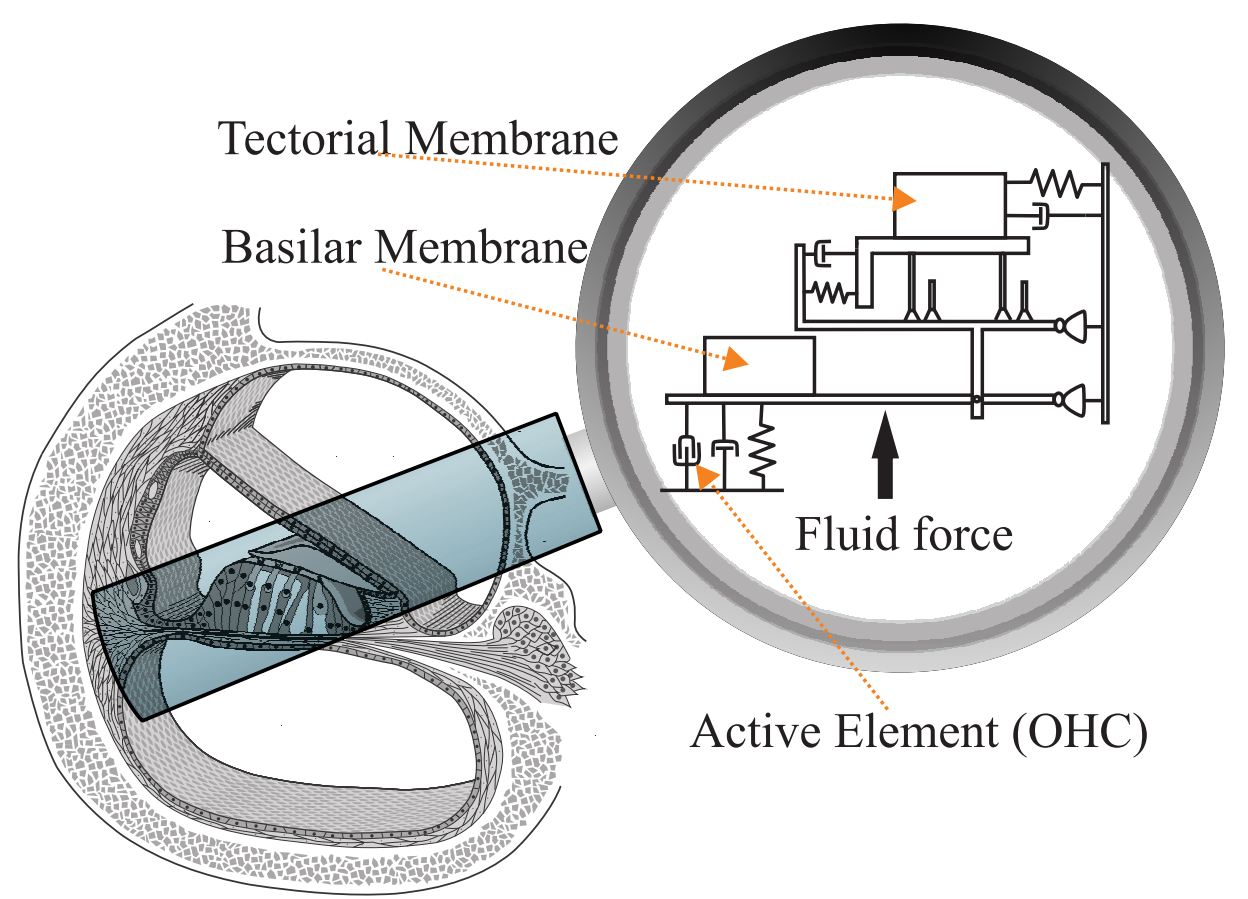

Figure 3.2: Neely and Kim's model. In this model different parts of the organ of Corti have been modelled by masses, springs and dashpots. The micromechanics model affects the boundary conditions of the macromechanics model. Redrawn Figure 4.5 from (Patuzzi, 1996) with permission.

maintained by the electrogenic pumping of potassium by stria vascularis (Patuzzi, 2011).

Vibrations of the basilar membrane deflect the stereocilia and modulate these flows of ions which are called MET currents.

For investigating the properties and effects of these standing and alternating flows of ions, the cochlea can be modelled as a network of biological resistances, capacitances, and voltage and current sources; i.e., an electrical model. 


\subsubsection{Electrical Modelling of the Organ of Corti}

The electrical properties of the organ of Corti without hair cells are similar to other biological tissues of the human body and can be modelled as a passive electrical network. However, the existence of the hair cells gives special electrical properties to this sensory epithelium and affects its mechanical behaviours. The IHC transduces mechanical vibration into neural stimulation which is sent to the brain for interpretation. The $\mathrm{OHC}$ nonlinearly amplifies the small basilar membrane motions, and this action consequently enhances the sensitivity of IHC to weak stimuli and compresses high level stimuli. Both amplification and compression by the $\mathrm{OHC}$ enormously increase the dynamic range of hearing (Fettiplace and Hackney, 2006; Nam and Fettiplace, 2012; Russell, 2008). Even though both IHCs and OHCs are electrically active elements inside the organ of Corti, the OHCs are believed to have the dominant influence on the extra-cellular currents and potentials (Withnell, 2001). The mutual interactions between mechanical and electrical parts of the organ of Corti caused by the OHCs have significant effects on the cochlear function and must be incorporated in a realistic cochlear model.

The Battery and variable resistance model by Davis (1965) is an initial attempt to model the electrical network properties and the distribution of potentials in the cochlea. In this model, the resting potentials of the cochlea have been modelled by two batteries; the primary battery is in the hair cells, an accessory battery is in the stria vascularis, and the MET channels are modelled by variable electrical resistors. Accordingly, the current through the hair cells is modulated by changing electrical resistances in accordance with cilia deflection. Strelioff (1973) suggested a network model of the resistors and batteries to simulate the generation and distribution of the cochlear potentials (Fig. 3.4). The results of this model were in agreement with previous physiological findings.

In Dallos $(1983,1984)$ electrical properties of the organ of Corti have been investigated. The electrical configuration and component values of the resulting detailed models for a radial section of the organ of Corti have been determined heuristically based on actual measurements of potentials inside the cochlea. Some 


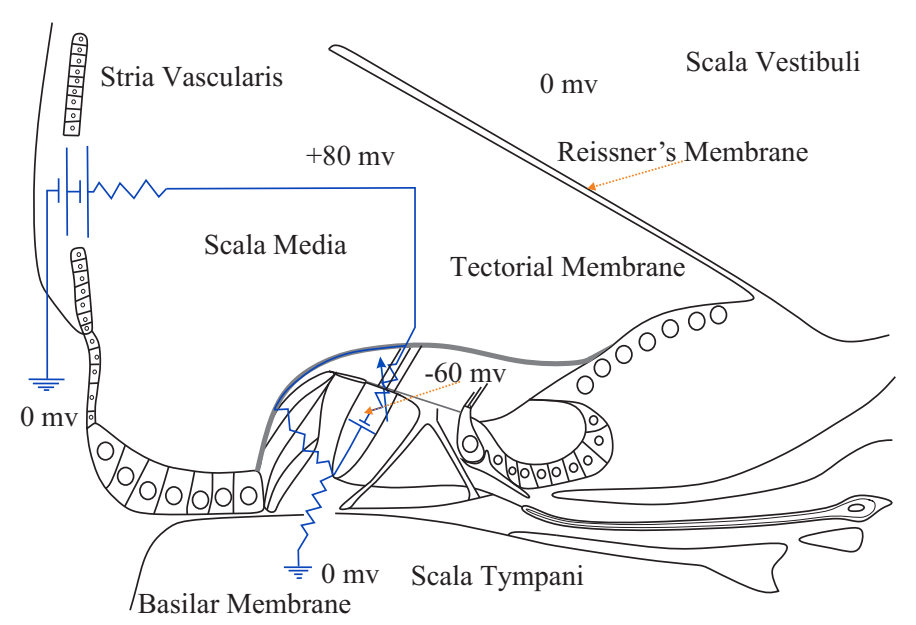

Figure 3.3: The battery or variable resistance model was initially proposed by Davis (1965) and has been widely used to model electrical activities inside the cochlea. According to this model the primary voltage source $(-60 \mathrm{mv})$ is intercellular potential, and the accessory voltage source $(+80$ $\mathrm{mv}$ ) is located in the stria vascularis. Source: from Davis (1965) with permission.

of these parameter values have been revised later by other researchers (Johnson et al., 2011). Even though electrical coupling has been rarely amalgamated into the cochlear models, some studies can be found in the literature.

In Ramamoorthy et al. (2007) a model has been proposed which integrates the electrical, mechanical and acoustical elements of the cochlea. This model provides a framework to successfully predict and reproduce the response of the cochlea to acoustical stimulus. Nonlinear characteristics of the MET channel and HB motility have not been considered in this model. A notable observation from this model is that longitudinal electrical coupling actually sharpens the mechanical response. Whether this effect is significant in a non-linear cochlea is not known. Nonlinear saturation behaviour of the MET channel has been incorporated in Liu and Neely (2010) to explore distortion product otoacoustic emission. In this model the longitudinal electrical connection in the organ of Corti and the hair bundle motility has been neglected. Nam and Fettiplace $(2010,2012)$ have used a mechanical model along with electrical coupling to investigate the effects of HB motility and the cochlear amplifier in high and low auditory frequencies. 


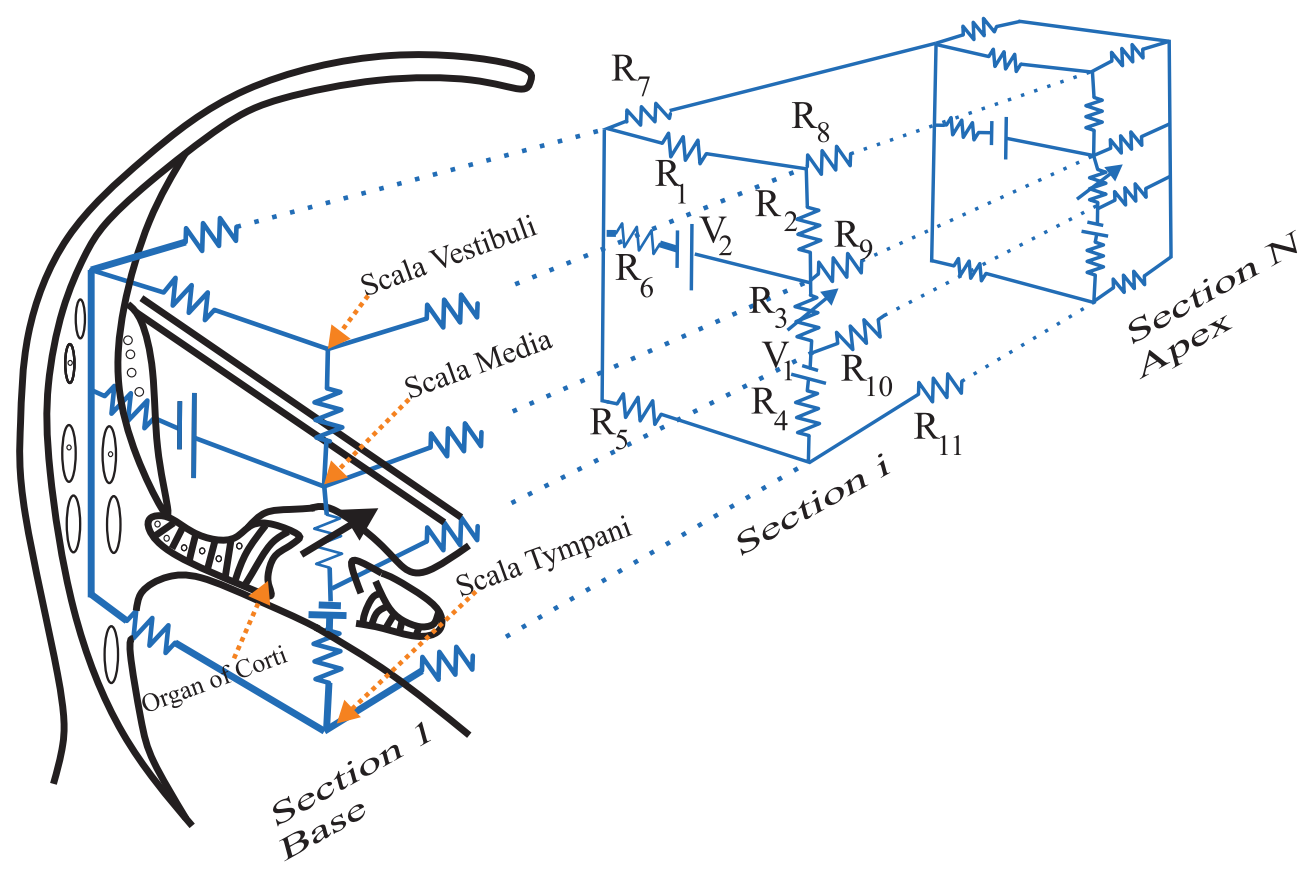

Figure 3.4: Strelioff's Network model. In this model, the cochlea is considered as an assembles of cross section slices. Each slice has six transverse resistors (R1-R6) and five longitudinal resistors (R7-R11). The parameter values of this model have been widely used by other investigators in this area. (redrawn from Strelioff (1973) with permission of J. Acoust. Soc. Am.)

Electrical properties of hair cells in vitro and in vivo have been thoroughly examined, and very sophisticated models with detailed ion channels can be seen in the literature (O'Beirne, 2005; O'Beirne and Patuzzi, 2007). A simple model of the IHC and OHC is presented in Fig. 3.5. This model or slightly different versions of it have been widely used in the area of modelling the distribution of the cochlear biopotentials and their effects. 


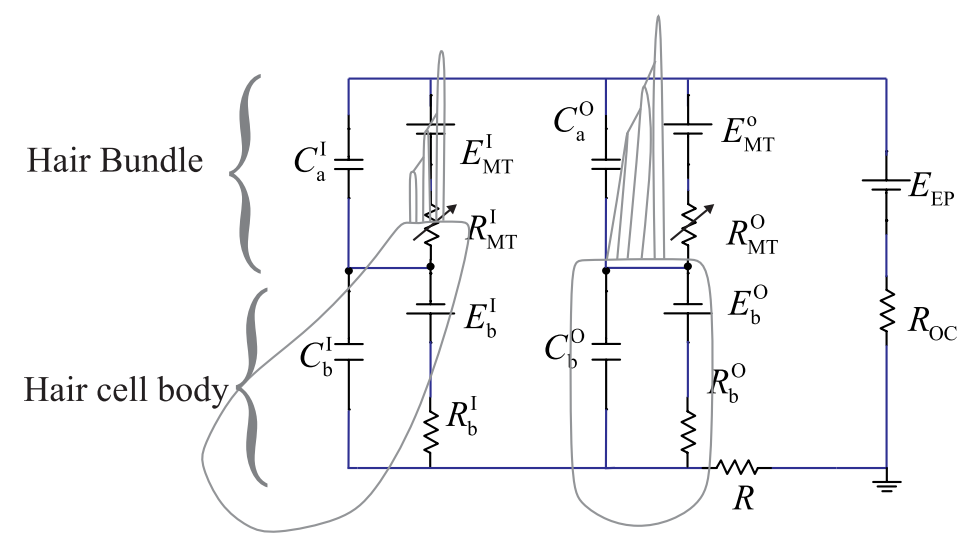

Figure 3.5: A circuit diagram for the inner and outer hair cells. Superscripts I and O denotes inner and outer hair cells. Subscripts MT, a and b indicate the MET channels, apical and basolateral parts of the hair cells, respectively. $R$ represents the overall resistance of the organ of Corti per single cross section to the ground. This model configuration or similar versions of it can be seen in Dallos (1983, 1984); Mistrík et al. (2009); Johnson et al. (2011); Cheatham et al. (2011) just to cite a few. Mistrík et al. (2009) has been used this electrical model with longitudinal resistances to model the current flow in a model of the cochlea.

\subsubsection{Cochlear Microphonic}

The cochlear microphonic (CM) is a by-product of cochlear electrical activity and probably can be considered as an epiphenomenon (Webster, 1973) ${ }^{5}$. The $\mathrm{CM}$ is an electrical signal which is easier to detect and measure than mechanical movement. von Békésy's electrophysiological measurements were aimed at locating the source of the $\mathrm{CM}$ and moreover he wanted to know the role of these electrical signals in sensing a tone (Guinan Jr et al., 2012).

As mentioned before, the cochlear amplifier causes the BM tuning curves to be sharply tuned at the characteristic frequency. However the $\mathrm{CM}$ which results from the BM vibration has broad tuning curves (Honrubia and Ward, 1968; Patuzzi,

\footnotetext{
${ }^{5}$ The CM might ameliorate the impact of OHC low cut-off frequency on the cochlear amplifier in higher frequencies (Dallos and Evans, 1995) which may bear on the effect of longitudinal electrical coupling observed by Ramamoorthy et al. (2007) which is mentioned on page 37. However this has been a subject of debate (Iwasa and Sul, 2008).
} 
1987). These discrepancies are thoroughly discussed in Chapter 5

\subsection{Modelling the Generation of the $\mathrm{CM}$}

For modelling purposes, the positive feedback gain responsible for the amplification effect inside the cochlea is usually modelled as a (nonlinear) active damping gain which is driven by BM and tectorial membrane displacement and velocity and acts as an active force on the mechanical parts (Elliott et al., 2007; Neely and Kim, 1986). However, for observing the CM in a cochlear model, electrical components which represent the electrical behaviour of the cochlea and construct this positive feedback process should also be incorporated in the model. In the models of Mistrík et al. (2009) and Cheatham et al. (2011) the electrical part of the model is analysed separately using mechanical data without considering physical links between the mechanical and electrical parts. Simplified versions of these physical links have been considered in some proposed models but investigating the CM was not the main objective of those models (Ramamoorthy et al., 2007; Liu and Neely, 2010; Nam and Fettiplace, 2010, 2012).

In the following, an integrated detailed model of the electrical and mechanical properties of the cochlea is presented. This integration allows bidirectional interaction between the mechanical and electrical aspects of the model. None of the components and configurations of the proposed model are original but these configurations have never been integrated for investigating the cochlear microphonic.

In a modified version of the model of Neely and Kim (1986), simplified electrical components without any longitudinal coupling also has been considered (Liu and Neely, 2010). In this model, the nonlinearity of mechanoelectrical transduction current of the OHCs is also considered. We have chosen the model of Liu and Neely (2010) as a foundation for our model. The details of this model, including nonlinearity, make this model suitable for our purpose. To model the generation of the $\mathrm{CM}$ and observe the behaviours of the $\mathrm{CM}$, a more realistic electrical coupling 
lumped model is amalgamated with the model of Liu and Neely (2010). We proceed now to briefly describe that model and then highlight the modification that we made to model the CM.

\subsubsection{The Model of Liu and Neely (2010).}

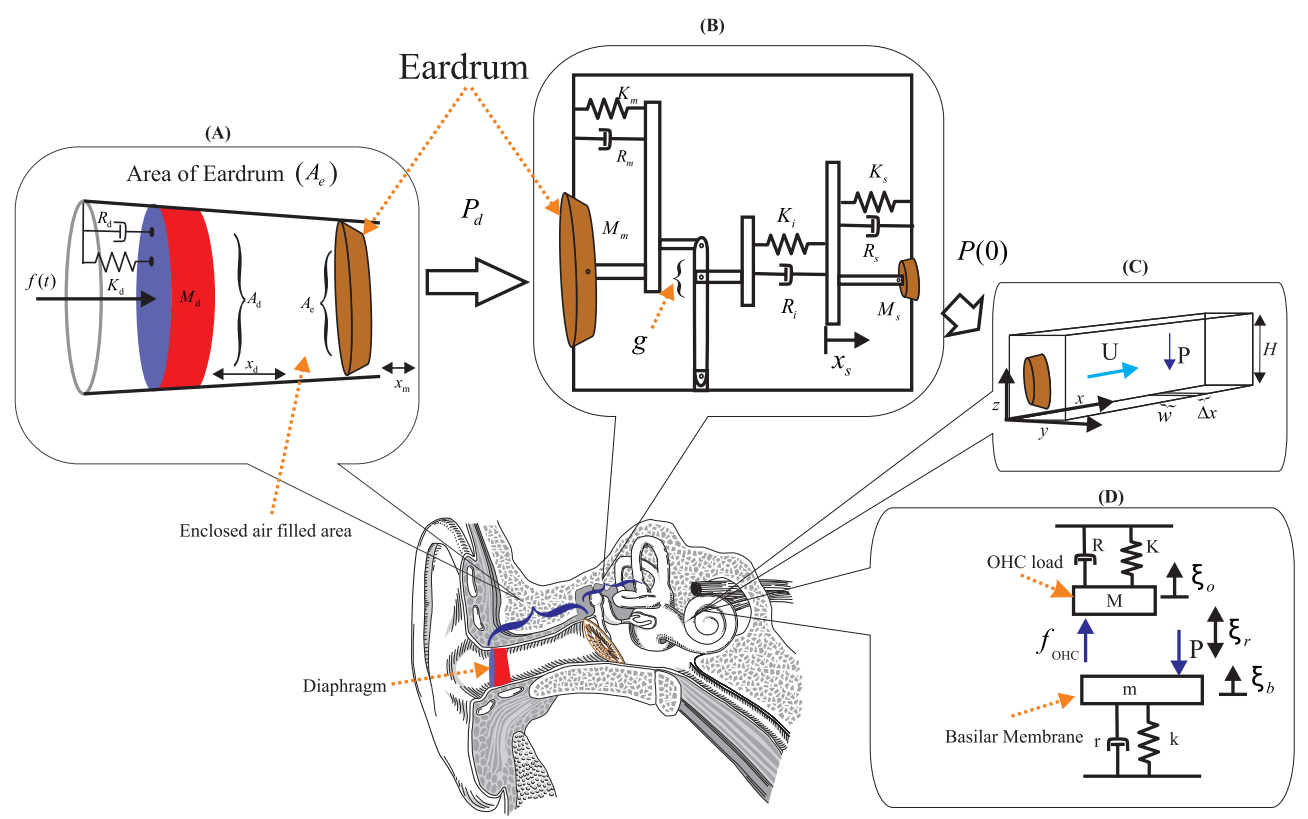

Figure 3.6: Liu and Neely's Model. (A) shows the model of the diaphragm which can be considered to be an earphone diaphragm made of rubber for prevention of air leakage. The diaphragm can be modelled by a mass-spring-damper system. (B) shows the model of the middle ear. Redrawn from Liu and Neely (2010) with permission of J. Acoust. Soc. Am. The middle ear transfers energy of sound to the fluid inside the cochlea and can be modelled with a lever and the mass-spring damper systems. (C) shows the macromechanics model of the cochlea. Simplified form of the Navier-Stokes equations can be used to describe the macromechanics model of the cochlea. (D) shows the micromechanics model of the cochlea. The organ of Corti can be mechanically can be considered as an array of mass-spring-damper systems with an active force which is induced by OHCs.

The mechanical part of our model is similar to the model of Liu and Neely (2009, 2010) to which the reader is referred for more details. For the integrity of our 
current work we repeat some of the equations and definitions from Liu and Neely (2009, 2010). We also provide more information on some parts of this model and show that the equations are consistent with the common ground laid out in the beginning of this chapter.

Liu and Neely's model is summarised in Fig.3.6 . Parameters $\left\{M_{\mathrm{d}}, R_{\mathrm{d}}, K_{\mathrm{d}}\right\}$ represent the sound source (which is here assumed to be the diaphragm of an earphone). The dynamics of the diaphragm are described by the following equation:

$$
M_{\mathrm{d}} \dot{v}_{\mathrm{d}}=f(t)-K_{\mathrm{d}} x_{\mathrm{d}}-R_{\mathrm{d}} v_{\mathrm{d}}+P_{\mathrm{d}} A_{\mathrm{d}}
$$

where $\dot{v}_{\mathrm{d}}, v_{\mathrm{d}}$ and $x_{\mathrm{d}}{ }^{6}$ denote the acceleration, velocity and displacement of the earphone diaphragm, respectively. $P_{\mathrm{d}}$ is the pressure in the enclosed space of the ear canal, and $A_{\mathrm{d}}$ is the area of the earphone diaphragm. $f(t)$ is the stimulus force on the earphone diaphragm(see Fig. 3.6 (A)).

The earphone diaphragm and the eardrum are coupled via the enclosed air filled ear canal between them. This air filled area can be modelled by an acoustic compliance $K_{\mathrm{c}}$, resulting in the following equation:

$$
P_{\mathrm{d}}=K_{\mathrm{c}}\left(x_{\mathrm{d}} A_{\mathrm{d}}-x_{\mathrm{m}} A_{\mathrm{e}}\right)
$$

where $x_{\mathrm{m}}$ is the displacement of the malleus (equal to the displacement of the eardrum. See Fig. 3.6 and Fig. 2.1), and $A_{\mathrm{e}}$ is the area of the eardrum.

\section{Model of the Middle Ear}

The eardrum-malleus-incus system is modelled by parameters $\left\{M_{\mathrm{m}}, R_{\mathrm{m}}, K_{\mathrm{m}}\right\}$. The malleus-incus lever ratio is $g \leq 1$. The joint between the incus and the stapes is modelled by parameters $\left\{R_{\mathrm{i}}, K_{\mathrm{i}}\right\}$. The stapes and its surrounding structures are modelled by parameters $\left\{M_{\mathrm{s}}, R_{\mathrm{s}}, K_{\mathrm{s}}\right\} . P_{\mathrm{d}}$ and $P(0)^{7}$ are coupled to each other via

\footnotetext{
${ }^{6}$ Henceforth, when $x$ denotes displacement, $v$ and $\dot{v}$ will denote velocity and acceleration, respectively.

${ }^{7} P(0)$ is the pressure at the stapes which is equal to the cochlea fluid pressure at the oval window.
} 
the following equations:

$$
\begin{gathered}
M_{\mathrm{m}} \dot{v}_{\mathrm{m}}=-K_{\mathrm{m}} x_{\mathrm{m}}-R_{\mathrm{m}} v_{\mathrm{m}}+g f_{\mathrm{i}}+P_{\mathrm{d}} A_{\mathrm{e}} \\
\left(M_{\mathrm{s}}+M_{\mathrm{r}}\right) \dot{v}_{\mathrm{s}}=-\left(K_{\mathrm{s}}+K_{\mathrm{r}}\right) x_{\mathrm{s}}-\left(R_{\mathrm{s}}+R_{\mathrm{r}}\right) v_{\mathrm{s}}-f_{\mathrm{i}}-P(0) A_{s} \\
f_{\mathrm{i}}=K_{\mathrm{i}}\left(x_{\mathrm{s}}-g x_{\mathrm{m}}\right)+R_{\mathrm{i}}\left(v_{\mathrm{s}}-g v_{\mathrm{m}}\right)
\end{gathered}
$$

$A_{\mathrm{S}}$ is the effective area of the stapes footplate; $x_{\mathrm{S}}$ and $v_{\mathrm{S}}$ denote the displacement and the velocity of the stapes. Parameters $\left\{M_{\mathrm{r}}, R_{\mathrm{r}}, K_{\mathrm{r}}\right\}$ represent the round window (See part (B) of Fig. 3.6.).

\section{Cochlear Macromechanics}

Equations (3.1), (3.2) and (3.3) govern cochlear macromechanics. These equations can be simplified to one dimension. By neglecting dependency on the $y$ direction (see Fig. 3.6), equation (3.1) can be simplified to

$$
\partial_{x} u_{x}+\partial_{z} u_{z}=0
$$

where $x$ denotes the longitudinal direction from base to apex, and $z$ denotes the vertical direction. $u_{x}$ and $u_{z}$ are components of the fluid velocity $u$ in these directions.

Assuming that the fluid velocity linearly changes from a maximum value at $z=0$ to zero at $z=H$ and the height of channel $(H)$ is assumed to be constant along and across the width of the BM (see Fig. 3.6 (C)), $u_{z}$ can be written as (Patuzzi, 1996):

$$
u_{z}=\frac{\dot{\xi}_{\mathrm{r}}(x)(H-z)}{H}
$$

and consequently the following relation can be derived:

$$
\frac{\partial}{\partial x} u(x, t)=\frac{1}{H} \dot{\xi}_{\mathrm{r}}
$$

where $\dot{\xi}_{\mathrm{r}}$ is the velocity of the reticular lamina. By replacing the velocity $u$ with volume velocity $U^{8}$ and considering cross-sectional area $A=H w$ in equations

$$
{ }^{8} U=A u \text {. }
$$


(3.7) and (3.2), the one dimensional equations for cochlear macromechanics follow:

$$
\begin{gathered}
\partial_{x} P=-\frac{\rho}{A} \dot{U} \\
\partial_{x} U=w \dot{\xi}_{\mathrm{r}}
\end{gathered}
$$

where $P$ denotes the pressure difference between the two cochlear compartments (scala vestibuli and scala tympani), $\rho$ is the density of the cochlear fluid, and $w$ is the width of the BM.

For calculating the boundary condition at the basal end, equations (3.8) and (3.9) can be combined:

$$
\partial_{x} P=-\frac{\rho}{H} \ddot{\xi}_{\mathrm{r}}
$$

Since at the basal end, the cochlear fluid moves with the same motion as the stapes (Neely, 1985), (3.10) yields the boundary condition at the base:

$$
\left.\partial_{x} P\right|_{x=0}=-\rho \dot{v}_{\mathrm{s}}
$$

By using the acoustic impedance of a tube the boundary condition at the helicotrema can be approximated as follows (Puria and Allen, 1991; Liu and Neely, 2010):

$$
\left.\partial_{x} P\right|_{x=L}=\frac{-\rho}{A m_{\mathrm{h}}} P(L)
$$

where $m_{\mathrm{h}}$ represents the mass of the fluid at the helicotrema (refer to Puria and Allen (1991); Liu and Neely (2010) for more details). It is noteworthy that in some cochlear models the boundary condition at the helicotrema is considered to be $P(L, t)=0$ (short circuit), or $P(L, t)=\infty$ (open circuit). The open circuit assumption is physiologically implausible since it does not permit any connection between the two scalae (Puria and Allen, 1991).

\section{Micromechanics Formulation}

Each section of the micromechanical part of the model consists of two parts representing the basilar membrane and OHCs load respectively as shown in 
part (D) of Fig. 3.6. By applying Newton's second law to each section of the micromechanical model, we obtain:

$$
\begin{aligned}
f_{\mathrm{OHC}} & =M \ddot{\xi}_{o}+R \dot{\xi}_{o}+K \xi_{o} \\
-P & =m \ddot{\xi}_{b}+r \dot{\xi}_{b}+k \xi_{b}
\end{aligned}
$$

where $m, r$, and $k$ are the mass, resistance, and stiffness of the basilar membrane per unit area, and $M, R$, and $K$ are the mass, resistance, and stiffness of the OHC load impedance. $\xi_{\mathrm{b}}, \dot{\xi}_{\mathrm{b}}$ and $\ddot{\xi}_{\mathrm{b}}$ denote the displacement, velocity and acceleration of the BM, respectively. $\xi_{\mathrm{o}}, \dot{\xi}_{\mathrm{o}}$ and $\ddot{\xi}_{\mathrm{o}}$ are the displacement, velocity and acceleration of the $\mathrm{OHC}$, respectively. The $\mathrm{BM}$ displacement is equal to the sum of the displacements of RL and OHCs $\left(\xi_{\mathrm{b}}=\xi_{\mathrm{r}}+\xi_{\mathrm{o}}\right) . \quad P$ is the fluid pressure difference between the scala vestibuli and scala tympani. $f_{\mathrm{OHC}}$ is the force induced by $\mathrm{OHC}$ electromotility.

\section{Electromotility}

Reduction in the length of the $\mathrm{OHC}$ accumulates charge $Q$, linearly related to OHC contraction displacement:

$$
\xi_{\mathrm{o}}=T Q
$$

where $T$ is a piezoelectric constant (refer to Section 2.4.2 and (Mountain and Hubbard, 1994)). This electrical charge produces an electrical current $i_{\mathrm{Q}}=\frac{d Q}{d t}$ and therefore,

$$
i_{\mathrm{Q}}=\frac{\dot{\xi}_{\mathrm{o}}}{T}
$$

$Q$ is a nonlinear function of

$$
\tilde{V}=V_{\mathrm{OHC}}-T f_{\mathrm{OHC}}
$$

and $C_{\mathrm{g}}=\partial Q / \partial \tilde{V}$. The force $\left(f_{\mathrm{OHC}}\right)$ is induced by $\mathrm{OHC}$ electromotility. $C_{\mathrm{g}}$ is a gaining capacitance. It can be assumed that $C_{\mathrm{g}}$ is constant and $C_{\mathrm{g}}=Q / \tilde{V}$ (Liu and Neely, 2009, 2010). 


\section{Mechanoelectrical Transduction}

In Liu and Neely's model, mechanoelectrical transduction (MET) current is described as follows:

$$
i_{\mathrm{r}}=I\left(\alpha_{\nu} \dot{\xi}_{\mathrm{r}}+\alpha_{\mathrm{d}} \xi_{\mathrm{r}}\right)
$$

where $I(\cdot)$ is a nonlinear function. $\xi_{\mathrm{r}}$ and $\dot{\xi}_{\mathrm{r}}$ represent the displacement and velocity of the reticular lamina. $\alpha_{\mathrm{v}}$ and $\alpha_{\mathrm{d}}$ are two coefficients which are defined as the MET's sensitivity to reticular lamina velocity and displacement. $I(\cdot)$ is defined as antisymmetric function:

$$
I(\eta)=I_{\max }\left(\frac{1}{1+\exp \left(-4 \eta / I_{\max }\right)}-\frac{1}{2}\right)=\frac{I_{\max }}{2} \tanh \frac{2 \eta}{I_{\max }}
$$

where $\eta=\alpha_{\mathrm{v}} \dot{\xi}_{\mathrm{r}}+\alpha_{\mathrm{d}} \xi_{\mathrm{r}}$, and $I_{\max }$ is the maximum range of OHC receptor current. For relatively small sound levels, MET current is approximately linear. For analysing the model in frequency domain in the next chapter, the linear form of MET current (i.e, $i_{\mathrm{r}}=\eta$ ) is used.

\subsubsection{Electrical Lumped Model of The Organ of Corti}

We here deviate from and extend the model of Liu and Neely (2010) and consider a more realistic electrical configuration of the organ of Corti in order to model the CM.

The organ of Corti electrically can be discretised into separate radial sections, each of which models a set of parallel OHCs (Strelioff, 1973; Ramamoorthy et al., 2007; Mistrík et al., 2009; Cheatham et al., 2011), and connections between adjacent sections via longitudinal cochlear fluid spaces. Each section can be modelled by passive electrical elements, independent and dependent electrical sources (Dallos, 1983, 1984; Mistrík et al., 2009).

A realistic electrical lumped model of a radial section of the organ of Corti is presented in Fig. 3.7.

The connection between extracellular and intracellular regions for both apical and 


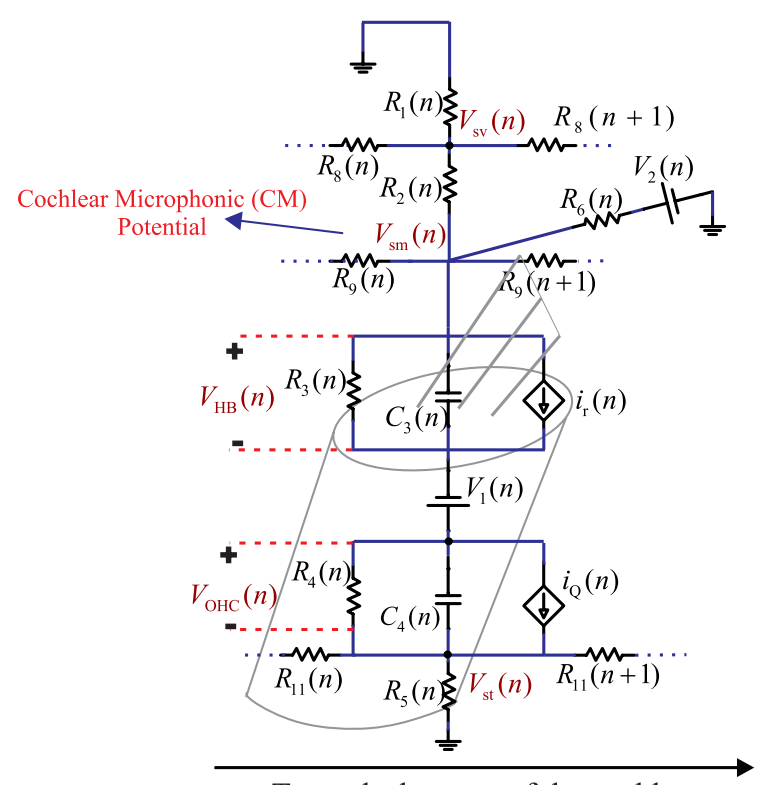

Towards the apex of the cochlea

Figure 3.7: The electrical networks model of the organ of Corti with dependent current sources and the longitudinal electrical coupling. The $\mathrm{CM}$ can be obtained as the potential of the endolymphatic space above the basal hair cells. Hence, $V_{\mathrm{sm}}$ represents the $\mathrm{CM}$ in the electrical model of the organ of Corti.

basal surfaces of the OHC can be modelled by a dependent current source, a capacitor and a resistor for each part. $i_{\mathrm{r}}$ is the dependent current source (MET channel current). $R_{3}$ and $C_{3}$ model the apical resistance and capacitance. $R_{4}$ and $C_{4}$ model basolateral resistance and capacitance. $i_{\mathrm{Q}}$ is the current associated with OHCs piezoelectricity. $V_{\mathrm{HB}}$ and $V_{\mathrm{OHC}}$ are the potentials of the hair bundle and OHC membrane, respectively.

The OHCs are embedded in the electrical networks of the organ of Corti. The electrical networks of the organ of Corti is modelled as six resistors. $R_{8}, R_{9}$ and $R_{11}$ represent the resistances along the scalae vestibuli, media and tympani respectively associated with the fluid resistances inside each compartment.

Note that the effect of the longitudinal resistor which models conduction along the tunnel of corti (for example $R_{10}$ in the network model of Strelioff (1973)) 
is ignored in the proposed model since this large resistance does not change the results noticeably.

$R_{1}, R_{5}$ and $R_{6}$ represent the resistance between each of these scalae and the surrounding spiral ligament which is considered to be ground $(0 \mathrm{~V})$ in the proposed model. More explanation of these elements and choosing their parameter values are provided in Appendix C.

The constant electrical potential provided by the stria vascularis and the resting membrane potential for OHCs are presented by $V_{2}(n)$ and $V_{1}(n)$ respectively.

$V_{\mathrm{sv}}, V_{\mathrm{sm}}$ and $V_{\mathrm{st}}$ are the potentials of the scala vestibuli, scala media and scala tympani, respectively. The potential $V_{\mathrm{sm}}$ can be approximated as the sum of the potentials $V_{\mathrm{OHC}}$ and $V_{\mathrm{HB}}$. The $\mathrm{CM}$ can be observed as the potential of the endolymphatic space above the basal hair cells ( $V_{\mathrm{sm}}$ in Fig. 3.7)(Mistrík et al., 2009).

Kirchhoff's circuit laws can be applied to these circuits to calculate the voltages and currents of each node and branch.

\subsection{Summary}

Even though improvements in observation and measuring techniques can reveal much information about the cochlea, there remain significant gaps between what can be measured and actual cochlear function. Therefore modelling plays an essential role in developing a better understanding of this remarkable organ. Cochlear models can be used to gain useful information and insights to categorise our understanding about this complex and sophisticated organ.

There is a wide variety of mechanical models that can be produced by using different assumption about the structure within the cochlea. Cochlear macromechanics is usually simplified to a one-dimensional transmission line model. Cochlear micromechanics is modelled by masses, springs, and dashpots (dampers).

The electrical processes and electrical components which are involved in the 
OHCs generating mechanical force are usually ignored in the cochlear models. However it is important to consider the electrical processes and electrical components in modelling the generation of the $\mathrm{CM}$.

The mechanical part of the introduced model is similar to the model of Liu and Neely (2010) and is summarised in Fig. 3.6.

In the introduced model, the $\mathrm{OHC}$ mechanoelectrical transduction current $\left(i_{\mathrm{r}}\right)$ is modelled as a nonlinear function of the RL velocity and displacement. The OHC length changes are based on the charge that transfers across the basolateral membrane and produces current $\left(i_{\mathrm{Q}}\right)$ in the proposed model. $i_{\mathrm{r}}$ and $i_{\mathrm{Q}}$ are represented by two dependent current sources (Fig. 3.7).

The proposed model is described by the equations (3.4) to (3.18) and Kirchhoff's circuit laws for the circuit shown in Fig. 3.7. In the next chapter, the solution methods of these equations are discussed.

\subsection{Contributions}

- A comprehensive and informative review of the existing literature on electrical couplings was presented.

- A realistic electrical representation of a section of the organ of Corti is amalgamated into an existing one-dimensional (1D) model of the cochlea by which the CM can be assessed. 
CHAPTER 3. MODELLING 


\section{Chapter 4}

\section{Methods of Solution and Model Validation}

Before performing any test on the model or explaining any observation based on the model, the model should be validated. In the previous chapter, the proposed model was described in detail. In this chapter, the mechanical and electrical results of the proposed cochlear microphonic model (PCM) are closely examined and compared objectively with pertinent experiments and other similar models. Mechanical behaviours of the cochlea are not directly within the scope of this work, but, for validating the model, mechanical outcomes should also be in a good agreement with relevant experiments.

To be used, a model must be formulated and solved. For a cochlear model, several approaches including numerical and analytical methods have been suggested (Steele and Taber, 1979; Ni, 2012).

The state space representation of a cochlear model is a simple way to apply and analyse a nonlinear cochlear model (Elliott et al., 2007; Moleti et al., 2009; Bertaccini and Sisto, 2011).

The state-space representation is explained in Section 4.1. We then justify the model using the results of the frequency domain analysis based on the state-space 
formulation in Section 4.2. Finally, we perform time domain analysis on the PCM and validate it in the time domain.

\subsection{State Space Representation of the Model}

Models usually consist of a set of inputs and outputs. Relations between inputs and outputs of physical models are usually described by differential equations. Therefore, they can be simply formulated as a state-space system. The state-space representation of a model can be readily extended to characterise nonlinearity in the model. More importantly, the state of a system can be completely described by a minimal set known as the state vector (Khoo, 2000) and all other unknown variables can be calculated using this vector.

The state-space approach for the proposed model is briefly presented here. More details on this approach for different cochlear models can be seen in Elliott et al. (2007) and Sisto et al. (2010).

The number of state variables is determined by the number of independent energy storage elements (Cha et al., 2000, Chapter 8). For example, in one section of the micromechanics part of the model (Fig.3.6 (D)), there are four independent energy storage elements, two mass elements and two spring elements. The mass elements store kinetic energy, and the spring elements store potential energy. The electrical part of the model (Fig. 3.7) has two capacitors as the independent energy storage elements for each section of the organ of Corti. The capacitors store energy in their electric field. Therefore for each section of organ of Corti six state variables should be considered.

For validating the model mechanically and investigating the $\mathrm{CM}$, the $\mathrm{BM}$ displacement, $\mathrm{OHC}$ and hair bundle voltages should be calculated. A state vector (minimal set) which can be defined to calculate these variables is the displacement and velocity of both the RL and the $\mathrm{OHC}$, together with $\mathrm{OHC}$ and hair bundle voltages 
in the organ of Corti:

$$
x_{n}=\left[\xi_{\mathrm{r}}(n), \dot{\xi}_{\mathrm{r}}(n), \xi_{\mathrm{o}}(n), \dot{\xi}_{\mathrm{o}}(n), V_{\mathrm{OHC}}(n), V_{\mathrm{HB}}(n)\right]^{\mathrm{T}}
$$

where $n$ indexes the discrete section of the cochlea.

By calculating $x_{n}$, the BM displacement and the CM can be then calculated ( $\xi_{\mathrm{b}}(n)=\xi_{\mathrm{r}}(n)+\xi_{\mathrm{OHC}}(n)$ and $V_{\mathrm{SM}} \simeq V_{\mathrm{OHC}}(n)+V_{\mathrm{HB}}(n)$, refer to Fig. 3.6 and 3.7). The BM is discretised into $N$ sections. Therefore the set of $N$ state vectors plus six more variables representing the state of the diaphragm and the middle ear ${ }^{1}$ (see Fig. 3.6) (A) and (B)) creates a system with a $6 N+6$ length state vector $\mathbf{x}$. The system can be represented in state-space form as:

$$
\begin{aligned}
\dot{\mathbf{x}}(t) & =\mathbf{A x}(t)+\mathbf{B u}(\mathbf{t}) \\
\mathbf{y}(t) & =\mathbf{C x}(t)
\end{aligned}
$$

where $\mathbf{A}: \mathbb{R}^{6 N+6} \mapsto \mathbb{R}^{6 N+6}$ is a state vector operator. The input to the model is a single input stimulus force $f(t)$ (see Fig. 3.6), and so $\mathbf{B u}(\mathbf{t})$ can be simply expressed as $[f(t), 0, \cdots, 0]^{\mathrm{T}}$.

The system output $\mathbf{y}(t)$ can be defined as any set of desired state variables which are selected by $\mathbf{C}: \mathbb{R}^{6 N+6} \mapsto \mathbb{R}^{k}$. For a linearized form of the model (if $i_{\mathrm{r}}=$ $\alpha_{\mathrm{v}} \dot{\xi}_{\mathrm{r}}+\alpha_{\mathrm{d}} \xi_{\mathrm{r}}$ ), the operators $\mathbf{A}, \mathbf{B}$ and $\mathbf{C}$ are matrices.

The state operator $\mathbf{A}$ can be written as the sum of four operators that correspond to 1) the earphone diaphragm, the eardrum and the middle ear $\left.\left(\mathbf{A}_{\mathrm{ME}}\right), 2\right)$ the micromechanics $\left.\left(\mathbf{A}_{\mathbf{C}}\right), 3\right)$ the fluid pressures $\left(\mathbf{A}_{\mathbf{P}}\right)$ and 4$)$ the electrical coupling $\left(\mathbf{A}_{\mathrm{G}}\right)$.

\section{Earphone Diaphragm, Eardrum and Middle ear $\left(A_{M E}\right)$}

The first six elements of state vector $\mathbf{x}$ are $\left[x_{\mathrm{d}}, v_{\mathrm{d}}\right]$ which represent the displacement and velocity of the earphone diaphragm (Liu and Neely, 2010)

\footnotetext{
${ }^{1}$ Eight energy storage elements model the diaphragm and the middle ear but only six of them are independent. The state of the air-filled area is linearly dependent on $x_{\mathrm{m}}$ and $x_{\mathrm{d}}$ (equation (3.5)) and the state of the join between the incus and the stapes is linearly dependent on $x_{\mathrm{m}}$ and $x_{\mathrm{s}}$ (equation (3.6c)).
} 
and $\left[x_{\mathrm{m}}, v_{\mathrm{m}}, x_{\mathrm{s}}, v_{\mathrm{s}}\right]$ which represent the displacement and velocity of the malleus-incus and stapes-oval window system (see Fig. 3.6 (A) and (B)). The first six rows and six columns of $\mathbf{A}_{\mathrm{ME}}$ are the coefficients of these state variables after writing equilibrium equations for the components of Fig. 3.6 (A) and (B) (Equations (3.4) to (3.6c) ) and all other elements of this matrix are zeros.

\section{Micromechanics $\left(\mathbf{A}_{\mathbf{C}}\right)$}

Using some algebraic manipulation equations (3.13), (3.14) and (3.16) can be rewritten as follows:

$$
\begin{aligned}
\frac{d \xi_{\mathrm{r}}}{d t} & =\dot{\xi}_{\mathrm{r}} \\
\frac{d \dot{\xi}_{\mathrm{r}}}{d t} & =\frac{R \dot{\xi}_{\mathrm{o}}+K \xi_{\mathrm{o}}}{M}-\frac{f_{\mathrm{OHC}}}{M}-\frac{r\left(\dot{\xi}_{\mathrm{r}}+\dot{\xi}_{\mathrm{o}}\right)+k\left(\xi_{\mathrm{r}}+\xi_{\mathrm{o}}\right)}{m}-\frac{P}{m} \\
\frac{d \xi_{\mathrm{o}}}{d t} & =\dot{\xi}_{\mathrm{o}} \\
\frac{d \dot{\xi}_{\mathrm{o}}}{d t} & =-\frac{R \dot{\xi}_{\mathrm{o}}+K \xi_{\mathrm{o}}}{M}+\frac{f_{\mathrm{OHC}}}{M}
\end{aligned}
$$

From (3.15) and (3.17), the force generated by the $\mathrm{OHC}$ is given by:

$$
f_{\mathrm{OHC}}=\frac{V_{\mathrm{OHC}}}{T}-\frac{\xi_{\mathrm{o}}}{C_{\mathrm{g}} T^{2}}
$$

Using equations (4.4a) to (4.4d) for $N$ parts results in $4 \times N$ state equations which can be used to construct matrix $\mathbf{A}_{\mathrm{C}}$. The fluid pressure $P$ is accounted for equation 4.4b using $\mathbf{A}_{\mathbf{P}}$.

\section{Fluid pressures (Ap)}

Fluid pressure acting on the BM (see Fig. 3.6 (C)) can be expressed in terms of the state variables. By differentiating both sides of (3.8) with respect to $x$ and combining it with (3.9) we have:

$$
\partial_{x}^{2} P=-\frac{\rho}{A}\left(\partial_{x} \partial_{t} U-\partial_{t} U \frac{\partial_{x} A}{A}\right) \approx-\frac{\rho}{A} w \frac{d \dot{\xi}_{\mathrm{r}}}{d t}
$$


From equations (3.14) and (4.4b), we obtain:

$$
\left(\partial_{x}^{2}-\frac{\rho w}{m A}\right) P=-\frac{\rho}{A} w\left(\frac{R \dot{\xi}_{\mathrm{o}}+K \xi_{\mathrm{o}}}{M}-\frac{f_{\mathrm{OHC}}}{M}-\frac{r\left(\dot{\xi}_{\mathrm{r}}+\dot{\xi}_{\mathrm{o}}\right)+k\left(\xi_{\mathrm{r}}+\xi_{\mathrm{o}}\right)}{m}\right)
$$

By using a finite differential approximation, equation (4.7) can be approximated as:

$$
\frac{P(n+1)-2 P(n)+P(n-1)}{(\Delta x)^{2}}-\frac{\rho w(n)}{m(n) A(n)} P(n)=l(\cdot)
$$

where $l(\cdot)$ is the right side of (4.7), and $n(0<n<N)$ is the index of the section.

Applying the boundary condition at the base (equations (3.6b) and (3.11)), we obtain:

$$
\frac{P(1)-P(0)}{\Delta X}=-\rho \dot{v}_{\mathrm{s}}
$$

therefore:

$$
\begin{aligned}
\frac{P(1)}{\Delta x}-\left(\frac{1}{\Delta x}+\frac{\rho A_{\mathrm{s}}}{M_{\mathrm{s}}+M_{\mathrm{r}}}\right) P(0) & = \\
& \frac{\rho}{M_{\mathrm{s}}+M_{\mathrm{r}}}\left(\left(K_{\mathrm{s}}+K_{\mathrm{r}}\right) x_{\mathrm{s}}-\left(R_{\mathrm{s}}+R_{\mathrm{r}}\right) v_{\mathrm{s}}-f_{\mathrm{i}}\right)
\end{aligned}
$$

The boundary condition at the apex (equation (3.12)) gives:

$$
\left(\frac{1}{\Delta x}+\frac{\rho}{A(N) m_{h}}\right) P(N)-\frac{P(N-1)}{\Delta x}=0
$$

Equations (4.8), (4.9) and (4.10) can be rewritten in a matrix form as:

$$
\left(\mathbf{D}_{2}-\hat{\mathbf{D}}_{2}\right) P=\mathbf{L x}
$$

where the second derivative is approximated by $\mathbf{D}_{2}$ which is an $N \times N$ tridiagonal centred finite difference approximation matrix. $\hat{\mathbf{D}}_{2}$ is a $N \times N$ diagonal matrix with $\frac{\rho}{A} w$ on its diagonal:

$$
\hat{\mathbf{D}}_{2}=\operatorname{diag}\left(\frac{\rho}{A(n)} w(n)\right)
$$


The first and last row of the resulting matrix $\left(\mathbf{D}_{2}-\hat{\mathbf{D}}_{2}\right)$ should be modified considering the boundary conditions at the base and apex (4.9) and (4.10).

The matrix $\mathbf{L}$ is constructed using $\mathbf{A}_{\mathbf{C}}$. A coefficient matrix $\mathbf{P}_{\dot{\xi}_{\mathrm{r}}}$ is required to rearrange the pressure values to a format suitable for combination with equation (4.4b), noting that Pressure $P(x)$ only appears in state equations related to the velocity of the $\mathrm{RL} \dot{\xi}_{\mathrm{r}}$ :

$$
\mathbf{A}_{P}=\mathbf{P}_{\dot{\xi}_{\mathrm{r}}}\left(\mathbf{D}_{2}-\hat{\mathbf{D}}_{2}\right)^{-1} \mathbf{L}
$$

4. Electrical Coupling $\left(\mathbf{A}_{\mathbf{G}}\right)$ For obtaining equations related to $\mathrm{OHC}$ and hair bundle voltages nodal analysis has been used. Using Kirchhoff's laws for each node in the electrical model (Fig. 3.7) results in the following equations:

$$
\begin{aligned}
& \frac{V_{\mathrm{sv}}(n)-V_{\mathrm{sv}}(n-1)}{R_{8}(n)}+\frac{V_{\mathrm{sv}}(n)-V_{\mathrm{sv}}(n+1)}{R_{8}(n+1)}+\frac{V_{\mathrm{sv}}(n)}{R_{1}(n)}+\frac{V_{\mathrm{sv}}(n)-V_{\mathrm{sm}}(n)}{R_{2}(n)}=0 \\
& \frac{V_{\mathrm{sm}}(n)-V_{\mathrm{sm}}(n-1)}{R_{9}(n)}+\frac{V_{\mathrm{sm}}(n)-V_{\mathrm{sm}}(n+1)}{R_{9}(n+1)} \\
& +\frac{V_{\mathrm{sm}}(n)-V_{\mathrm{sv}}(n)}{R_{2}(n)}+\frac{V_{\mathrm{sm}}(n)-V_{2}(n)}{R_{6}(n)}+\frac{V_{\mathrm{HB}}(n)}{R_{3}(n)}+C_{3}(n) \dot{V}_{\mathrm{HB}}(n)=-i_{\mathrm{r}}(n)
\end{aligned}
$$

$$
\begin{gathered}
\frac{V_{\mathrm{st}}(n)-V_{\mathrm{st}}(n-1)}{R_{11}(n)}+\frac{V_{\mathrm{st}}(n)-V_{\mathrm{st}}(n+1)}{R_{11}(n+1)}+\frac{V_{\mathrm{st}}(n)}{R_{5}(n)} \\
-\frac{V_{\mathrm{OHC}}(n)}{R_{4}(n)}-C_{4}(n) \dot{V}_{\mathrm{OHC}}(n)=i_{\mathrm{Q}}(n) \\
V_{\mathrm{st}}(n)+V_{\mathrm{OHC}}(n)+V_{\mathrm{HB}}-V_{1}(n)-V_{\mathrm{sm}}(n)=0 \\
C_{3}(n) \dot{V}_{\mathrm{HB}}(n)+\frac{V_{\mathrm{HB}}}{R_{3}(n)}-\frac{V_{\mathrm{OHC}}(n)}{R_{4}(n)}-C_{4}(n) \dot{V}_{\mathrm{OHC}}(n)=-i_{\mathrm{r}}(n)+i_{\mathrm{Q}}(n)
\end{gathered}
$$


This is the only part of the model that is different for the linear and nonlinear versions. Note that by linearizing $i_{\mathrm{r}}$ to $\alpha_{\mathrm{v}} \dot{\xi}_{\mathrm{r}}+\alpha_{\mathrm{d}} \xi_{\mathrm{r}}$ (see page 46), the right side of these equations is made up of a combination of state variables in each row. These equations can be written in matrix form:

$$
\mathbf{G V}=\mathbf{E x}
$$

where

$$
V=\left[V_{\mathrm{st}}(1) V_{\mathrm{sm}}(1) V_{\mathrm{sv}}(1) \dot{V}_{\mathrm{OHC}}(1) \dot{V}_{\mathrm{HB}}(1) \cdots \dot{V}_{\mathrm{HB}}(N)\right]^{\mathrm{T}}
$$

E constructs the combination of the state variables of the right side of equations (4.13) to (4.17).

Matrix $\mathbf{K}$ can be made to extract the $2 \times N$ state variables $\dot{V}_{\mathrm{OHC}}$ and $\dot{V}_{\mathrm{HB}}$. Therefore:

$$
\mathbf{A}_{\mathrm{G}}=\mathbf{K ~ G}^{-1} \mathbf{E}
$$

Finally,

$$
\mathbf{A}=\mathbf{A}_{\mathrm{ME}}+\mathbf{A}_{\mathrm{C}}+\mathbf{A}_{\mathrm{P}}+\mathbf{A}_{\mathrm{G}}
$$

The details of the form of these matrices are given in Appendix A.

\subsection{Frequency Domain Analysis}

Taking the Fourier transform of both sides of (4.2) and (4.3), the frequency response of output $\mathbf{y}(j \omega)$ of the linearized form of the model, can be calculated as:

$$
\mathbf{y}(j \omega)=\mathbf{C}(j \omega \mathbf{I}-\mathbf{A})^{-1} \mathbf{B} \mathbf{u}(j \omega)
$$

where the variables of interest are extracted by the matrix $\mathbf{C}$. 


\subsubsection{Mechanical Responses}

To inspect the mechanical behaviour of the model, the variables of interest that matrix $\mathbf{C}$ extracts are $\xi_{\mathrm{r}}$ and $\xi_{\mathrm{o}}$. The $\mathrm{BM}$ displacement can then be calculated using the displacements of both $\mathrm{RL}$ and $\mathrm{OHC}^{2}$.

\section{Greenwood-map}

The peak location of the frequency tuning of the BM should be in agreement with the place-frequency map or Greenwood-map (Greenwood, 1990b) which is a general criterion for validating a cochlear model.

The Greenwood-map (or the Greenwood's function) associates a position on the $\mathrm{BM}$ to a characteristic frequency using the following mathematic relation:

$$
f=A\left(10^{a\left(x_{o}-x\right)}-k\right)
$$

where $f$ is the characteristic frequency (CF) and $x$ is the length from the base (i.e, the best place (BP)). Constant parameters, $A=165.4 \mathrm{~Hz}, a=0.06 \mathrm{~mm}^{-1}, x_{o}=35$ $\mathrm{mm}$ and $k=0.88$ approximate the place-frequency map of human cochleae (Greenwood, 1990b). The Greenwood's function is also used to determine electrode locations for cochlear implants (Darlene et al., 1998). Fig. 4.1 shows the place-frequency map of the model and Greenwood's function for humans. The model concurs reasonably with the place-frequency map for frequencies greater than $200 \mathrm{~Hz}(x<28.5 \mathrm{~mm})$. However for more apical parts, the placefrequency map of the model deviates from the place frequency map and reaches its maximum deviation at the most apical location, which is one of the limitations of the proposed model. Similar models ${ }^{3}$ such as the models of Ku (2008) and Liu and Neely (2010) suffer from the same limitation. In this thesis, we do not focus on the responses from specific part of the cochlea, therefore this mechanical shortcoming of the model does not change the presented results. Fig. 4.2 (a) shows

\footnotetext{
$2 \xi_{\mathrm{b}}=\xi_{\mathrm{r}}+\xi_{\mathrm{o}}$, see page 45 and Fig. 3.6 (D).

${ }^{3}$ Those models of the cochlea which model the micromechanical part with two masses.
} 


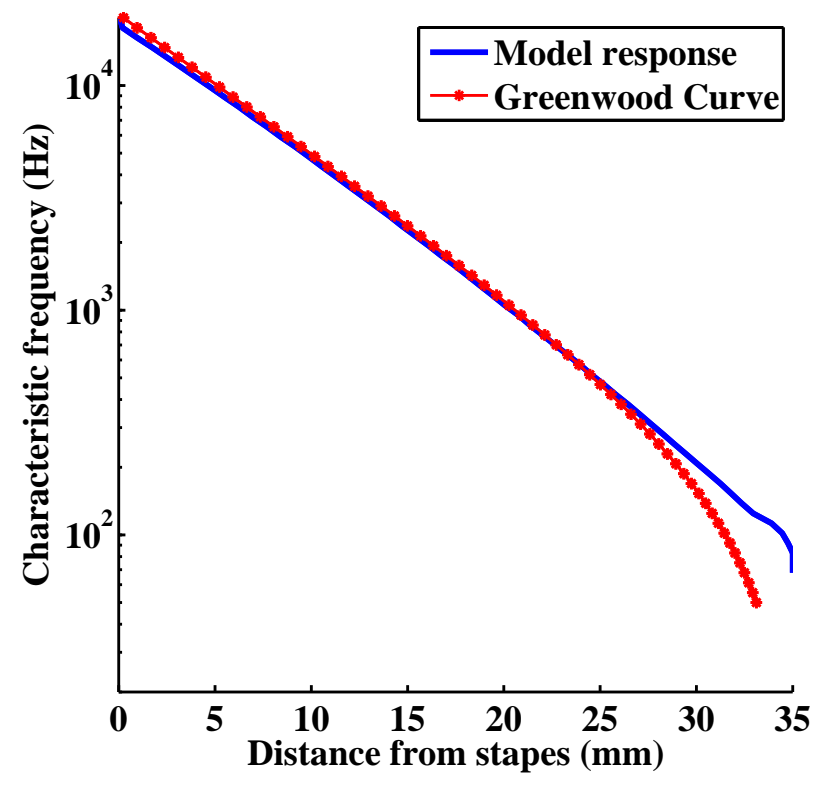

Figure 4.1: The place-frequency map of the model and the Greenwood curve for the human cochlea. For more apical parts, the place-frequency map of the model does not match the Greenwood curve. It is a shortcoming of the model.

the amplitudes and (b) shows the phases of the BM displacement in response to a $60 \mathrm{~dB}$ SPL pure tone stimulus at different locations along the cochlea.

\section{Cochlea amplifier}

A realistic model of the cochlea should be capable of providing realistic amplification. Fig. 4.2 (a) shows the results of the model for the amplitudes of the BM displacement in response to $60 \mathrm{~dB}$ SPL pure tone stimulus at different location along the cochlea. For demonstrating the amplification ability of the cochlear amplifier in the proposed model, the basilar membrane displacement tuning curve (for characteristic frequency equal to $1300 \mathrm{~Hz}$ ) with disabled amplifier has also been shown with a dotted line in Fig. 4.2 (a).

The cochlear amplifier in the proposed model is able to provide near 100 -fold amplification to the basilar membrane displacement induced by acoustic energy, 
(a)

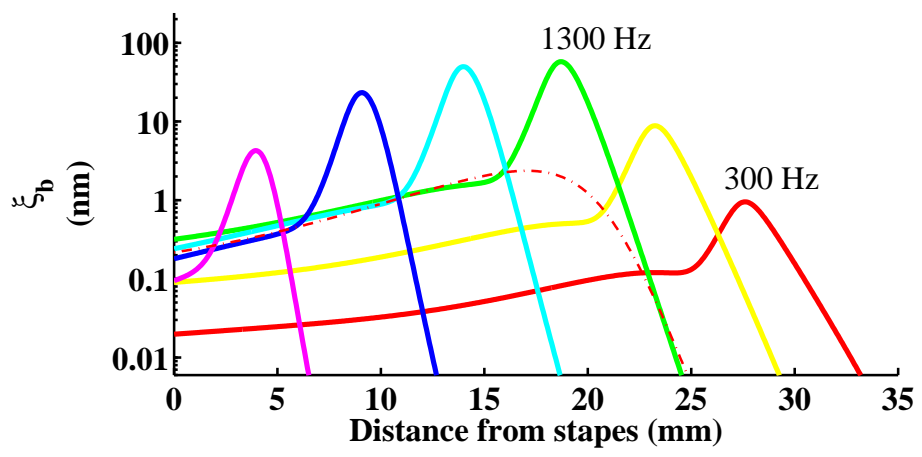

(b)

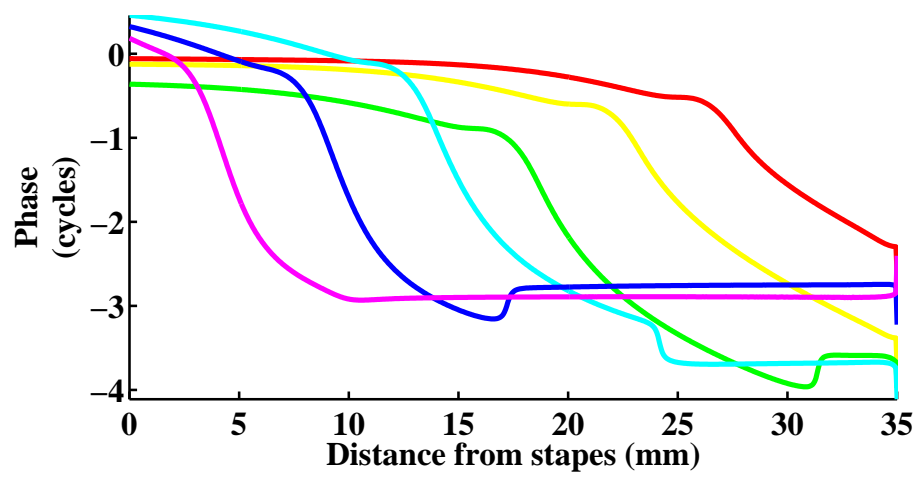

Figure 4.2: Mechanical responses of the model in the frequency domain. The basilar membrane displacement, as a function of the cochlear position. (a) shows the magnitude and (b) shows the phase of the basilar membrane displacement for six different stimulus frequencies: 10900, 5300, $2600,1300,600$ and $300 \mathrm{~Hz}$.

which is also in agreement with experiments reported in Müller and Gillespie (2008). The cochlear amplifier functions in a nonlinear way in response to the input stimulus. Here for frequency domain analysis, the cochlear amplifier is assumed to be working in the linear region. It should be noted that the amplitude of the stimulus is only relevant for the nonlinear region of the model (See Appendix C). For a comparison with other modelling results, Fig. 4.3 is redrawn from Fig. 5 of Neely and Kim (1986). Comparing Fig. 4.2 and Fig. 4.3 indicates that the proposed model gives a qualitatively similar results. 
Another feature which can be observed in both Fig. 4.2 (a) and Fig. 4.3 (a) is noticeable differences between the magnitudes of the BM displacement of the model for different locations. These differences in magnitude indicate that the middle region the cochlea is the most sensitive and sensitivity declines towards both apical and basal regions of the cochlea. This observation also qualitatively agrees with the equal loudness ${ }^{4}$ levels.

\section{Tuning}

For mammalian cochleae, BM vibrations are more peaked towards the base of the cochlea than those towards the apex (Robles and Ruggero, 2001). By inspecting the results of the model in Fig.4.2, it can be seen that BM tuning curves are more sharply tuned for higher frequencies (towards the base) than lower frequency (towards the apex).

The sharpness of tuning can be measured quantitatively by the quality factor (Q), which is the ratio of the centre frequency to the bandwidth. Experimental measurements of the BM tuning curves are not available for human. However the quality factor $(\mathrm{Q})$ can be estimated using psychoacoustical and modelling approaches. Three estimations of $\mathrm{Q}_{10 \mathrm{~dB}}$ i.e., centre frequency divided by bandwidth $10 \mathrm{~dB}$ below the peak, are reported in Ruggero and Temchin (2005) for humans based on different available animal models. To verify the BM tuning sharpness quantitatively, the $\mathrm{Q}_{10 \mathrm{~dB}}$ values of the proposed model are compared to the three aforementioned estimations of $\mathrm{Q}_{10 \mathrm{~dB}}$ and presented in Fig. 4.4.

It is noteworthy that higher $\mathrm{Q}_{10 \mathrm{~dB}}$ values have also been estimated (for example in Oxenham and Shera (2003)).

The observations of the model made in this section based on the frequency

\footnotetext{
${ }^{4}$ Loudness is a subjective perception of sound level. The intensities which are required in order for pure tones of different frequencies to be perceived equally loud are called equal loudness levels (Gelfand, 2010, Chapter 11). Comparison between loudness data and the BM displacement data demonstrates that the loudness curves is partly a reflection of the maximum amplitude of the basilar membrane vibration at each position along the cochlea (Buus and Florentine, 2002).
} 
(a)

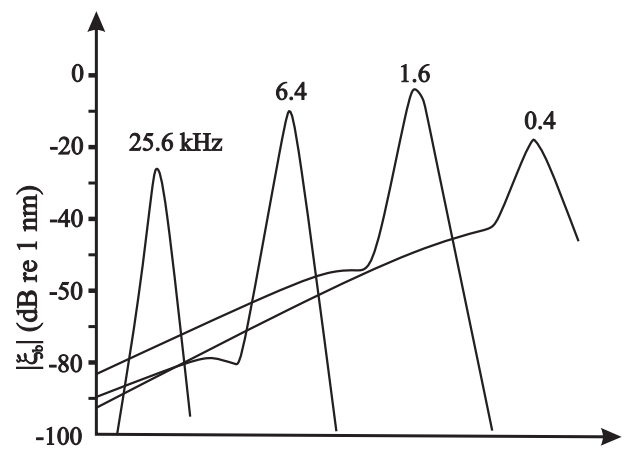

(b)

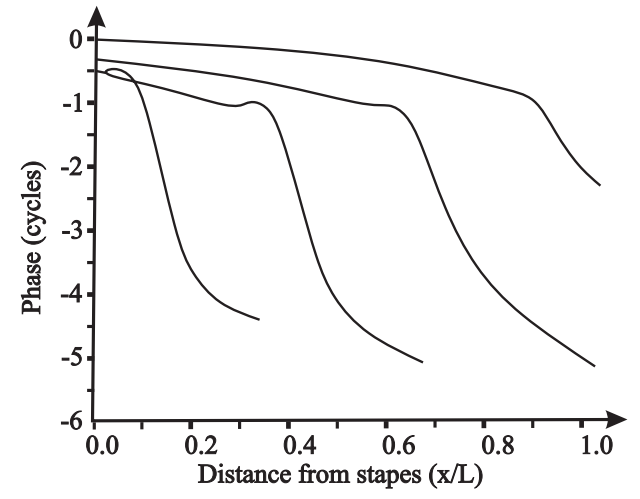

Figure 4.3: The basilar membrane displacement of the cat, as a function of the cochlear position. Reproduced from Fig. 5 of Neely and Kim (1986) with permission.

responses of the linearized model, indicate that the model is capable of producing realistic mechanical responses. The nonlinear growth of the BM displacement against the input intensity is assessed in Section 4.3 and Appendix C.

\subsubsection{Electrical Responses}

$V_{\mathrm{OHC}}$ and $V_{\mathrm{HB}}$ are the state variables for assessing the electrical responses of the model and the CM. Other scalae potentials can be calculated using $V_{\mathrm{OHC}}$ and $V_{\mathrm{HB}}$. Fig. 4.5 (a) depicts the amplitudes of these potential variables as a function of the 


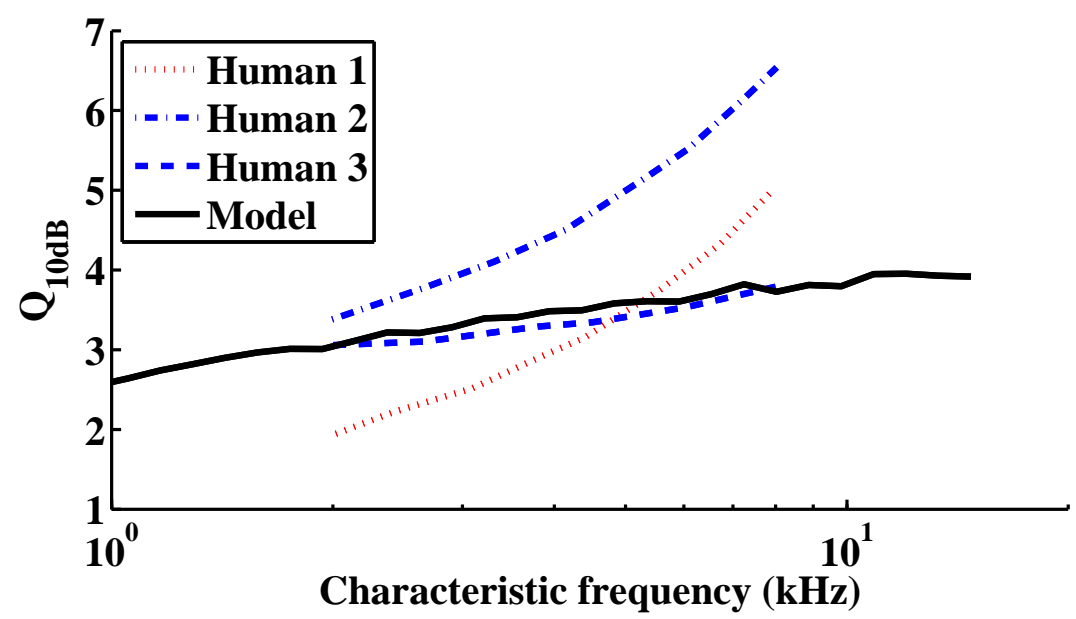

Figure 4.4: $\mathrm{Q}_{10 \mathrm{~dB}}$ for the $\mathrm{BM}$ tuning curves is shown. Data for Human 1, 2 and 3 are three different estimations of $\mathrm{Q}_{10 \mathrm{~dB}}$ for humans based on animal models, which are extracted from Fig. 5. of Ruggero and Temchin (2005). The results of the model are in a good agreement with Human 3 estimation and also with the values reported in Choi et al. (2008).

cochlear position. Fig. 4.5 (b) shows that unlike the sharp tuning curves of the BM, $V_{\mathrm{OHC}}$ and $V_{\mathrm{HB}}$ (see Fig. 4.2 (a) and Fig. 4.5 (a)), the $\mathrm{CM}\left(V_{\mathrm{sm}}\right.$ in Fig. 3.7) has broad tuning curves.

The discrepancy between the sharpness of the basilar membrane tuning curves and CM tuning curves can also be seen in experiments (for example measurements reported in Honrubia and Ward (1968) and Fridberger et al. (2004)) that qualitatively validates the CM results of the proposed model.

The Fig. 4.5 (a) clearly shows that $V_{\mathrm{OHC}}$ and $V_{\mathrm{HB}}$ are sharply tuned and very similar in amplitudes. However, they have a phase differences of $\pi$ radians or half cycle near their peaks (Fig. 4.6) which makes the summation (the CM) broadly tuned. The broadness of the CM tuning curves is investigated in detail in Chapter 5. 
(a)

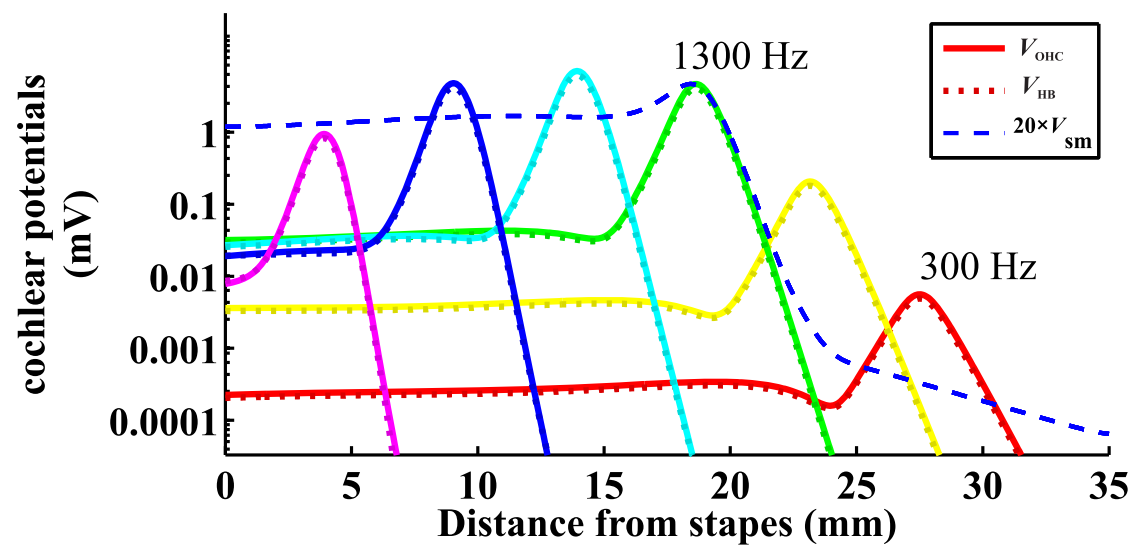

(b)

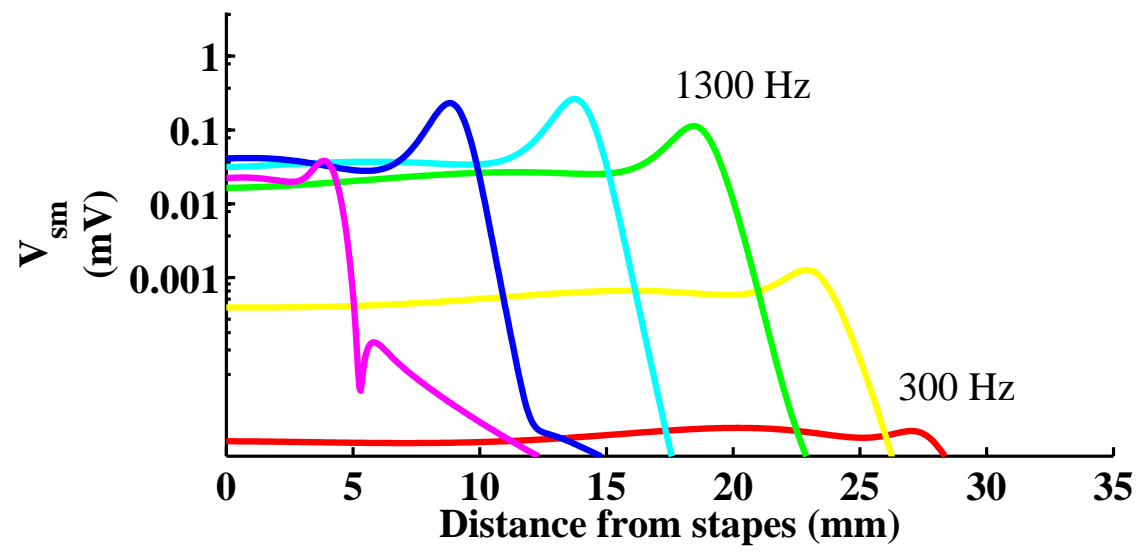

Figure 4.5: (a) illustrates the model's predicted amplitudes of $V_{\mathrm{OHC}}, V_{\mathrm{HB}}$ and (b) shows $V_{\mathrm{sm}}$ $\left(V_{\mathrm{sm}} \simeq V_{\mathrm{OHC}}+V_{\mathrm{HB}}\right)$ along the cochlea using frequency domain analysis of the model. Each graph shows the responses for six different stimulus frequencies, 10900, 5300, 2600, 1300, 600 and $300 \mathrm{~Hz}$. The scaled version (by 20) of the $\mathrm{CM}\left(V_{\mathrm{sm}}\right)$ tuning curve (with $\mathrm{CF}=1300 \mathrm{~Hz}$ ) is also shown in (a). As can be seen from panel (a), the amplitude of $V_{\mathrm{sm}}$ (the $\mathrm{CM}$ ) is less tuned than $V_{\mathrm{OHC}}$ and $V_{\mathrm{HB}}$. 


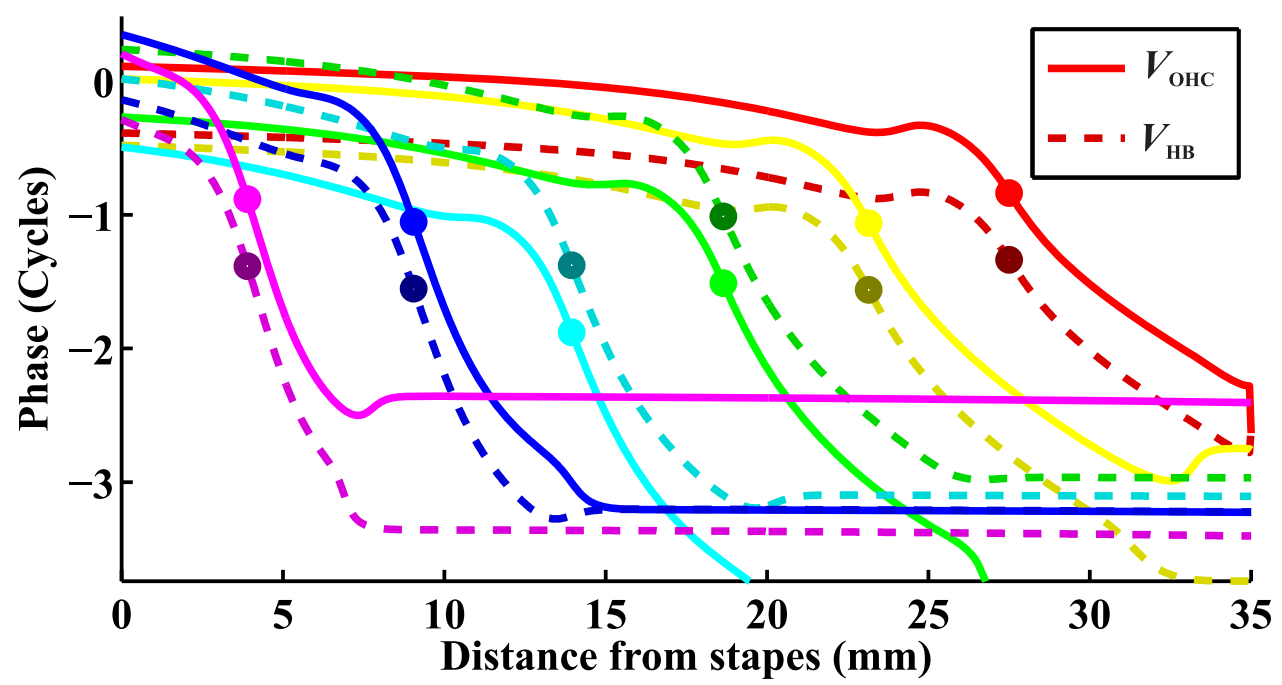

Figure 4.6: Phases of $V_{\mathrm{OHC}}$ and $V_{\mathrm{HB}} . V_{\mathrm{OHC}}$ and $V_{\mathrm{HB}}$ have a phase difference of $\pi$ near their peaks based on the proposed model. The peak locations have been marked by $\bullet$.

\subsection{Time Domain Analysis}

In order to explore the nonlinear behaviour of the model, the model should be analysed in the time domain. As stated earlier, the state space approach (Elliott et al., 2007; Sisto et al., 2010) provides a simple foundation for representation of a cochlear model and for analysis of the model using conventional numerical methods. The non-inverted form of matrices $\mathbf{D}_{2}$ and $\mathbf{G}$ are sparse in this method, so the speed of the numerical solution can be increased if these matrices can be kept in non-inverted form (Teal et al., 2011).

In general, solving the differential equations of the cochlear model is usually challenging and sometimes requires numerical techniques that are very specific to the problem type (Teal et al., 2011; Bertaccini and Sisto, 2011). For instance, the state vector of the proposed model consists of $6 N+6$ elements. For 5 OHCs in each row and each section (15 OHCs per section) $N$ is equal to 700 (see Section 4.1). This means a large system of ordinary differential equations of dimensions $4206 \times 4206$ should be solved. An attractive alternative is to use a circuit analogy. 
The differential equations of the system can be derived by equivalent circuits for the nonelectrical parts of the model. Representing a model with equivalent electrical circuits not only makes the model more comprehensible, but it also provides access to numerous powerful numerical tools that have been built for circuit analysis. In addition, it can be shown that the equations of the cochlear model are stiff (Bertaccini and Sisto, 2011) and SPICE uses various effective techniques (Yamamura et al., 2005) to deal with stiff differential equations, which makes it a suitable tool to analyse the cochlear model. The equivalent electric circuit method is explained in Appendix B. Appendix B also provides a framework to represent by electrical circuit any one-dimensional model of the cochlea, that can include nonlinearity. In the following section, time domain analysis is performed on the model, including nonlinearity, using circuit analogies.

\subsubsection{Response to a broadband Stimulus}

The cochlea functions as a real-time spectrum analyser and decomposes an acoustic input signal spatially along its length (Dallos, 1992) and as discussed in Section 4.2.1, the location of the maximum amplitude of the BM vibration is related to the frequency of the input. High frequency stimuli cause maximum vibrations at the proximal end of the cochlea while the low frequency stimuli cause maximum vibration at the distal end of the cochlea. A click can be considered as a summation of different pure tones (sine waves) with appropriate phases and amplitudes. Therefore, this broadband stimulus causes propagating vibrations with higher frequencies from the base and with lower frequencies towards the apex. The BM displacement responses to a click $(10 \mu s)$ at three positions along the chinchilla cochlea are shown in Fig. 4.7.

Fig. 4.8 shows results of a time domain analysis of PCM at three different locations along the cochlea for the basilar membrane displacement and voltages of the scala media. The propagation of the travelling wave can be seen in this figure. The longitudinal spatial pattern of the mechanical results concurs with experimental data which is shown in Fig. 4.7, and measurements of Recio et al. 


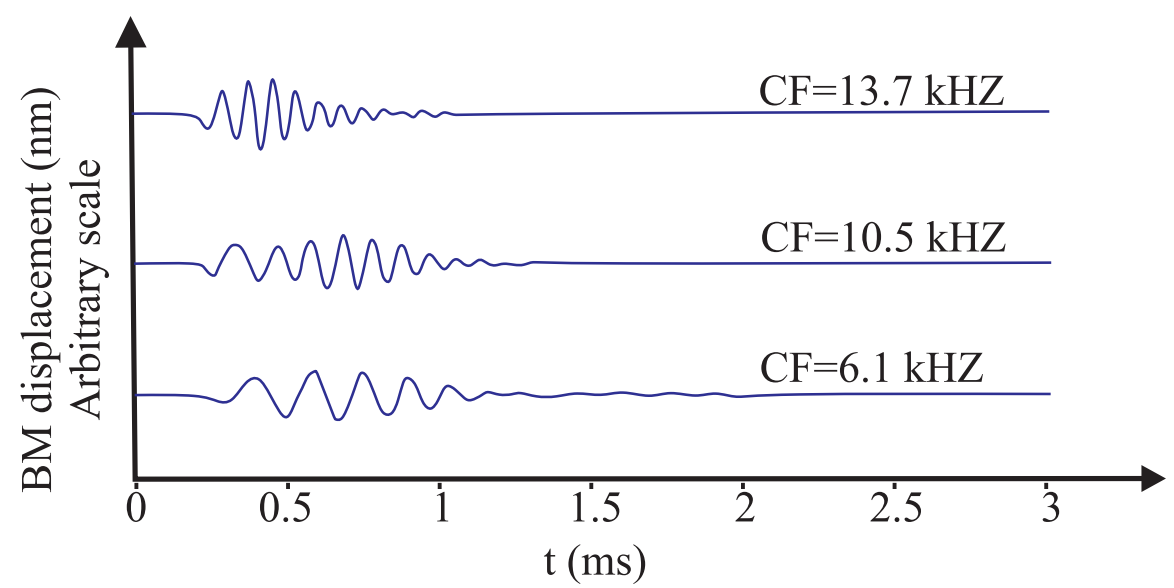

Figure 4.7: The BM responses to a $87 \mathrm{~dB}$ SPL click of duration $10 \mu s$. These responses were recorded at three positions a the chinchilla cochlea. This figure is reproduced from Fig. 7 of Recio and Rhode (2000) with permission. Note that in the Greenwood's function (4.22) constant parameters, $A=163.5 \mathrm{~Hz}, a=0.114 \mathrm{~mm}^{-1}, x_{o}=18.4 \mathrm{~mm}$ and $k=0.85$ represent the place-frequency map of the chinchilla cochlea (Greenwood, 1990b). Hence, the characteristic frequencies $6.1,10.513 .7 \mathrm{kHz}$ are associated with the locations $4.5,2.5$ and $1.5 \mathrm{~mm}$ from the base.

(1998) and Recio and Rhode (2000).

Fig. 4.9 depicts $V_{\mathrm{OHC}}$ and $V_{\mathrm{HB}}$ at one location. These responses are in agreement with the frequency analysis of the model showing that $V_{\mathrm{OHC}}$ and $V_{\mathrm{HB}}$ have nearly $\pi$ phase difference. More results of time domain analysis are addressed in Chapter 6.

\subsection{Effect of Cochlear Amplifier on the CM}

In vivo measurements show that in cochleae with prestin-null outer hair cells (i.e., deactivated cochlear amplifier), the $\mathrm{CM}$ amplitudes almost remain unchanged (Fig. 4.10) (Liberman et al., 2002; Cheatham et al., 2011). To probe this observation with the model, we consider the passive version of the proposed model in which $f_{\mathrm{OHC}}=0$ (Fig. 3.6) and consequently there is no $i_{\mathrm{Q}}$ in the coupling 
(a)

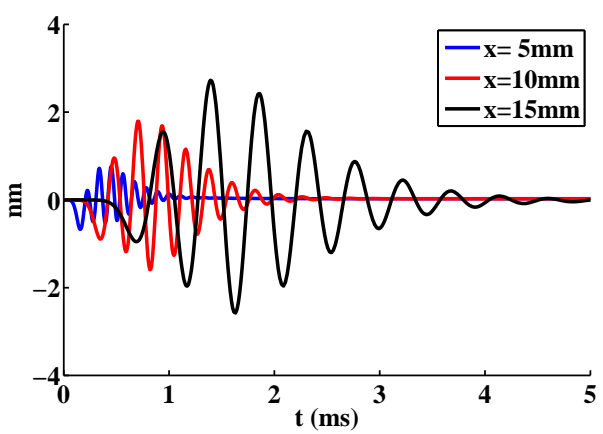

(c)

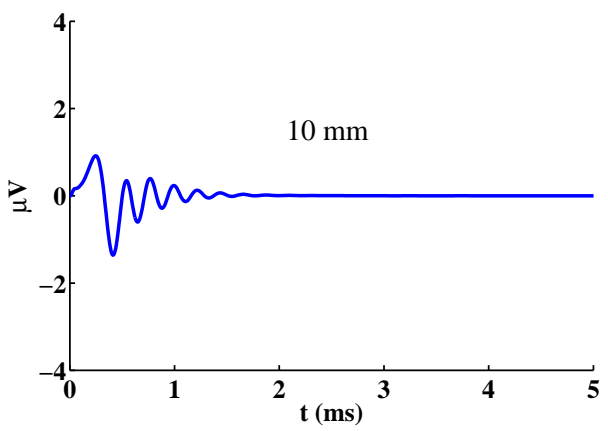

(b)

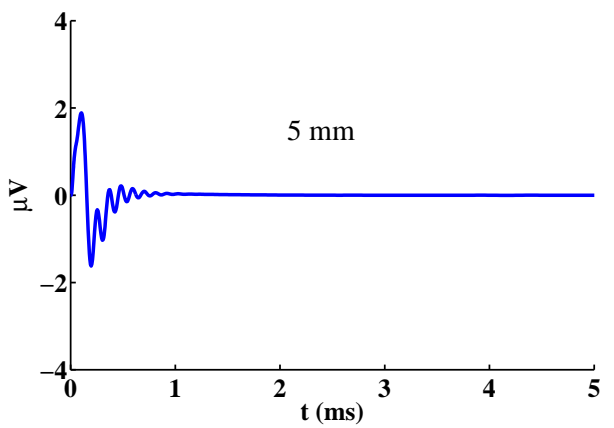

(d)

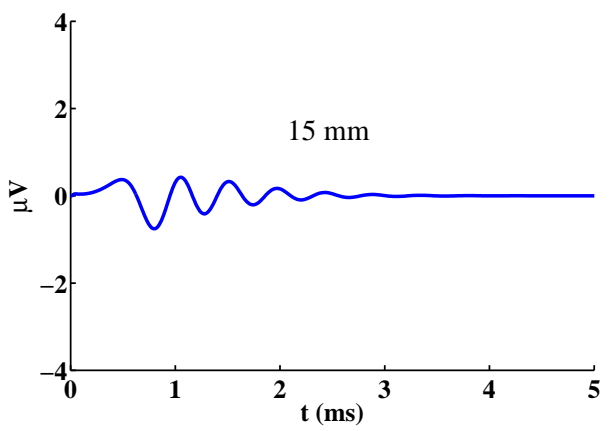

Figure 4.8: (a) shows the basilar membrane displacements at locations $5 \mathrm{~mm}$ (blue line), $10 \mathrm{~mm}$ (red line) and $15 \mathrm{~mm}$ (black line) from the stapes. (b), (c) and (d) show potetials of the scala media $\left(V_{\mathrm{sm}}\right)$ at locations 5,10 and $15 \mathrm{~mm}$ from the stapes respectively, in response to a $60 \mathrm{~dB}$ SPL click of duration $40 \mu \mathrm{s}$.

circuit (Fig. 3.7). Fig. 4.11 illustrates the time domain analysis for an active (i.e., active cochlear amplifier) system including nonlinearity in the MET channel current and a passive (i.e., silenced cochlear amplifier) system in respond to a 60 dB SPL pure tone stimulus of frequency $2000 \mathrm{~Hz}$. By inspecting Fig. 4.11 (b) and (c), the results show that the cochlear amplifier significantly amplifies the $\mathrm{BM}$ displacement while the effect on the $\mathrm{CM}$ is less significant, both of which agree with previous physiological findings (Liberman et al., 2002; Cheatham et al., 2011). Moreover, the results of the model exhibit post-stimulus ringing at the termination of the stimulus which is expected from the active cochlea and 


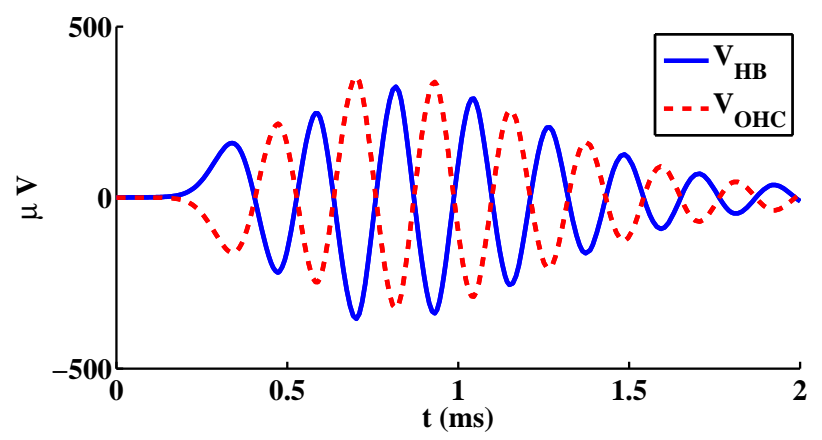

Figure 4.9: $V_{\mathrm{OHC}}$ and $V_{\mathrm{HB}}$ at location $10 \mathrm{~mm}$ from the stapes, in response to a click of duration 40 $\mu \mathrm{s}$.

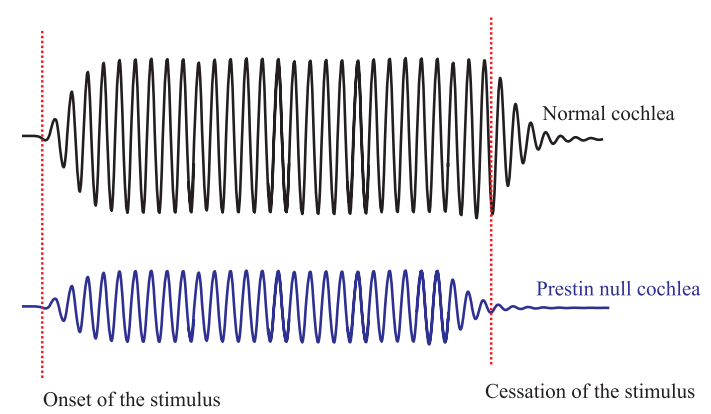

Figure 4.10: The CM of mouse cochleae with normal and prestin-null OHCs to a $80 \mathrm{~dB}$ SPL, $16 \mathrm{kHz}$ stimulus. The normal cochlea (i.e., the cochlea with the cochlear amplifier) produces "post-stimulus echo" whereas the cochlea with prestin-null OHCs does not. These waveforms are reproduced from Fig.4 of Liberman et al. (2002) with permission.

agrees with the physiological measurements of Liberman et al. (2002). The BM displacement and CM attenuate rapidly at the cessation of stimulus for the passive cochlea as suggested by the experimental measurements of Liberman et al. (2002); Kemp (1978b). 
(a)

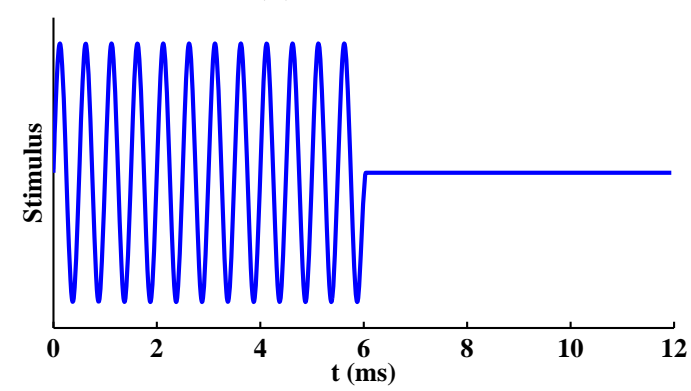

(b)

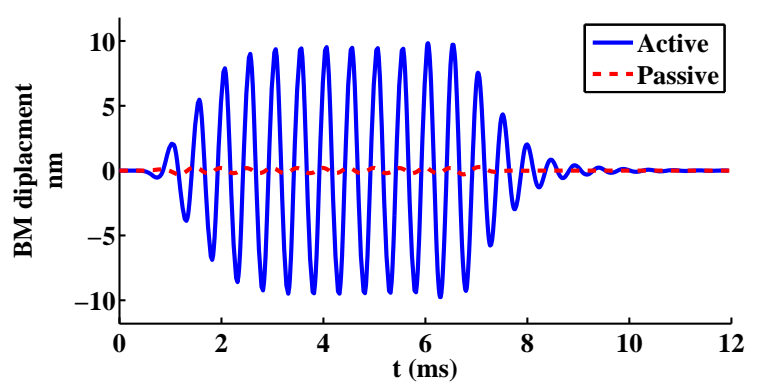

(c)

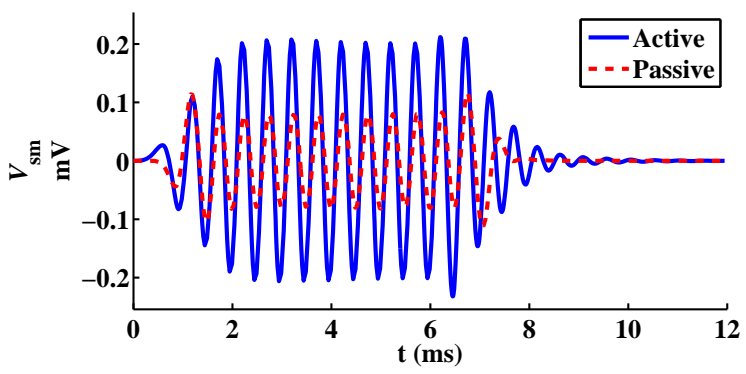

Figure 4.11: (a) Shows a $60 \mathrm{~dB}$ SPL pure tone stimulus of frequency $2000 \mathrm{~Hz}$. (b) shows the BM displacement for the passive and active models at the best place $(16.5 \mathrm{~mm}$ from the base for $\mathrm{CF}=2000 \mathrm{~Hz})$. (c) shows the $\mathrm{CM}\left(V_{\mathrm{sm}}\right)$ for passive and active model at the same place. 


\subsection{Summary}

The proposed model is discretised and represented in state-space form. For justifying the model, frequency domain analysis is first performed on the model. The mechanical responses of the model agree with Greenwood map. The model is capable of providing a realistic amplification gain at the best place. The BM tuning also qualitatively and quantitatively agrees with empirical experiments.

In addition, the results show that the $\mathrm{CM}$ exhibits broad tuning in contrast to the sharp tuning of the BM displacement. This also agrees with experiments. For analysing the model in the time domain, the proposed model is converted to an equivalent circuit. The results of the proposed model agree with $\mathrm{CM}$ measurements of both passive and active cochlea. The cochlear amplifier significantly affects the amplitudes of the basilar membrane displacement or velocity while it only slightly changes the amplitude of the CM. 


\subsection{Contributions}

- The model was validated based on mechanical and electrical results of the frequency and time domain analyses.

- A convenient framework was proposed to represent a cochlea model with electrical circuits by which the cochlear model can be readily analysed in circuit analysis software such as SPICE (see Appendix B too).

- Effect of the cochlear amplifier on the CM and BM displacement was investigated by the model. 


\section{Chapter 5}

\section{Cochlear Microphonic Broad Tuning Curves}

In Chapter 4, the model demonstrated sharp tuning curves for the BM displacement and broad tuning curves for the $\mathrm{CM}$ which are both in agreement with experiments.

A question in the interpretation of the $\mathrm{CM}$ is why the internally measured $\mathrm{CM}$ which is obtained by electrodes inserted in the scala media does not show the same degree of sharp frequency tuning as does the causative mechanical vibration measured at the same location. In other words, the $\mathrm{CM}$ is the results of mechanical vibration of the basilar membrane, therefore, the sharpness of the $\mathrm{CM}$ tuning curve could be expected to be similar to the sharpness of the BM tuning curves. The physiological measurements, however exhibit relatively broad tuning of the CM (Patuzzi, 1987). For example, the physiological measurements reported in Honrubia and Ward (1968); Dallos et al. (2005); Fridberger et al. (2004) show the discrepancy between tuning curves for the BM displacement and those for the $\mathrm{CM}$ (see Fig. 5.1).

The cochlear microphonic produced by pure tone stimulation is the vectorial average in the electrical network of the organ of Corti of the potentials from 
thousands of hair cells, each having a different phase and amplitude. This vectorial averaging causes longitudinal cancellation (i.e, the potential produced by the OHCs at one location in the cochlea can be cancelled by those produced at other locations in the cochlea) ${ }^{1}$ and has been considered to be the main cause of broadness in the $\mathrm{CM}$ tuning compared to the sharp tuning of the basilar membrane motion (Fridberger et al., 2004; Gelfand, 2010, Chapter 4).

To examine this hypothesis by the proposed model, we probe the effect of longitudinal cancellation on the $\mathrm{CM}$ in Section 5.2. The effect of phase cancellation in each cross-section of the organ of Corti is investigated in Section 5.3. Finally the effect of longitudinal resistances is discussed in 5.3.1.

\subsection{Broadness of the CM Tuning Curves}

Fig. 5.1 shows BM displacement and CM spatial tuning curves for the guinea pig cochlea. The $3 \mathrm{~dB}$ width of the $\mathrm{CM}$ is higher in comparison to the $3 \mathrm{~dB}$ width of the BM tuning curve.

Some researchers have suggested that the extracellular potential generated by hair cells in the best place can be cancelled by those in other locations (Dallos et al., 2005; Kletsky and Zwislocki, 1980; Patuzzi, 1987; Fridberger et al., 2004; He et al., 2012a; Gelfand, 2010, Chapter 4) and this is an often-cited justification for the broadness of CM tuning curves.

In situ recording of the functioning $\mathrm{OHCs}$ for investigating this hypothesis is exceptionally difficult. We use the model to investigate the cancellation hypothesis in the next section.

\footnotetext{
${ }^{1}$ Note that the BM consists of transverse beamlike fibres (see Section 3.2.1) and therefore in most cochlear models including our model the mechanical longitudinal coupling of the BM has been neglected, and the cochlear partition is modelled as transverse segments. However, the segments are coupled longitudinally via the cochlear fluids. Therefore, electrical responses generated at one location can be affected by those generated at other locations via electrical connection between them through the cochlear fluids.
} 


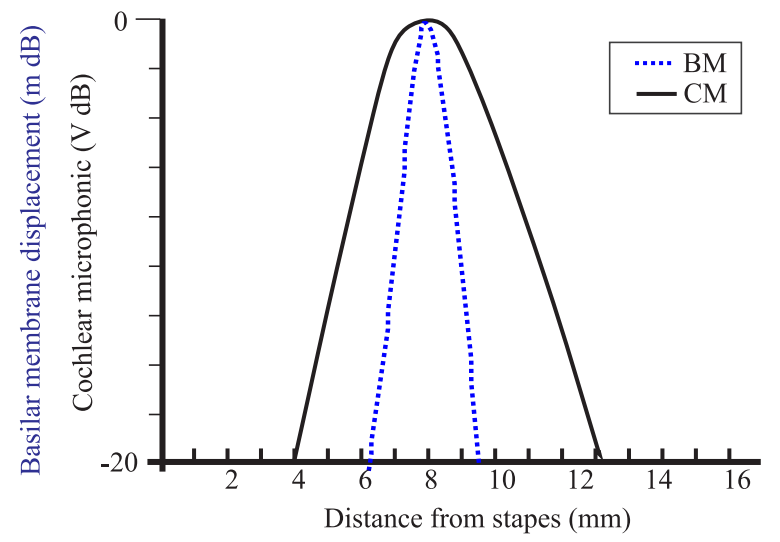

Figure 5.1: In mammals, the $\mathrm{CM}$ is broadly tuned in comparison to the sharply-tuned basilar membrane displacement. The amplitude of the $\mathrm{CM}$ recorded from scala media along the cochlea with micro-electrodes is shown with a solid line and the amplitude of the BM displacement spatial tuning curve is shown with a dotted line. This figure is produced using the CM measurement of the guinea pig (Honrubia and Ward, 1968) and the guinea pig modelling results of Neely and Kim (1986). The stimulus frequency for both curves is $2500 \mathrm{~Hz}$. Both amplitudes are normalised to their maximum values.

\subsection{Effect of Longitudinal Phase Cancellation on the CM Tuning Curves}

In the proposed cochlear microphonic model PCM, dependent current sources $\left(i_{\mathrm{r}}\right.$ and $\left.i_{\mathrm{Q}}\right)$ are derived from the displacement and velocity of the reticular lamina $\left(\xi_{\mathrm{r}}\right.$ and $\dot{\xi}_{\mathrm{r}}$ ), and the velocity of the $\mathrm{OHC}\left(\dot{\xi}_{\mathrm{o}}\right)$. Therefore by taking the values of these variables from the mechanical part of the model, the electrical part of each section of the organ of Corti can be analysed separately. We assume that an electrode can record the electrical signal from different locations along the cochlea.

For assessing the effect of phase cancellation on the broadness of the CM tuning curves, this four step procedure was followed:

Step I Analyse the PCM in the frequency domain for a stimulus with a specific frequency. 
Step II Save the reticular lamina velocity and displacement and OHC velocity $\left(\dot{\xi}_{\mathrm{r}}, \xi_{\mathrm{r}}\right.$ and $\left.\dot{\xi}_{\mathrm{o}}\right)$ for each section.

Step III Feed all or some subsets of these results into the electrical circuit.

Step IV Observe the effect on the potential of each section.

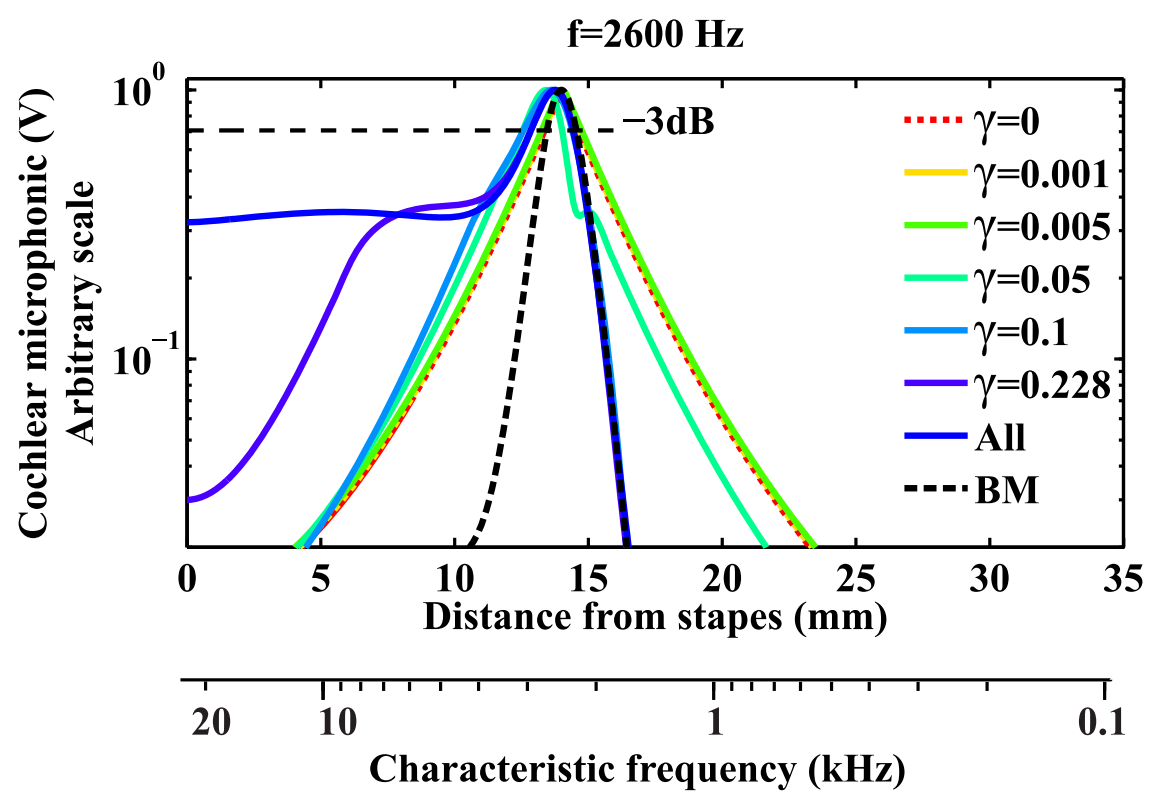

Figure 5.2: The CM spatial tuning curves for stimulus frequency of $2600 \mathrm{~Hz} . \quad \gamma=0$ means only the segment at the best place contributes and All means all segments contribute to the scala media potentials. BM is the basilar membrane displacement spatial tuning curve for the same stimulus frequency (dashed line), which is much sharper than the CM tuning curve even without longitudinal coupling (dotted line). The non-normalised amplitudes of the CM at the best place are illustrated in Fig. 5.4.

Frequency domain analysis was performed on the model for several stimulus frequencies (for example, 10900, 5300, 2600, 1300, 600 and $300 \mathrm{~Hz})($ Step I). The velocity and displacement of the reticular lamina and the velocity of $\mathrm{OHC}$ are three complex numbers with a different amplitude and phase for each radial segment (Step II). 
These complex numbers were used to set the electrical current sources for stimulating each section of the electrical part of the model. $\gamma$ is defined as the proportion of the total length of the cochlea ( $L=35 \mathrm{~mm}$ for the human cochlea) that is electrically active on each side of the best place (BP) associated with the CF according to the place-frequency map of human cochleae (see page 58). This means that if $d_{\mathrm{BP}}$ is the location of the best place, the region from $\max \left(0, d_{\mathrm{BP}}-\gamma L\right)$ to $\min \left(L, d_{\mathrm{BP}}+\gamma L\right)$ is contributing to the scala media potential at each side of the best place, in addition to the section of the best place. $i_{\mathrm{r}}$ and $i_{\mathrm{Q}}$ are equal to zero outside of this active region. $\gamma=0$ means only one section at the best place has nonzero current sources i.e., only this section has functioning OHCs (Step III).

Fig. 5.2 and Fig. 5.3 illustrate how longitudinal coupling changes the sharpness of the CM spatial tuning curves. The amplitude of the BM displacement is also shown with a dashed line in this figure. As can be seen, the $\mathrm{CM}$ even without longitudinal contribution of other OHCs (dotted line) is more broadly tuned than the BM displacement.

The visual assessment of Fig. 5.2 and Fig. 5.3 indicates that longitudinal coupling does not significantly change the sharpness of the CM spatial tuning curve. In addition, the curve for $\gamma=0$ illustrates that the CM decays exponentially, which agrees with measurements and modelling predictions of Kletsky and Zwislocki (1979); Mistrík et al. (2009).

Fig. 5.4 shows how longitudinal coupling affects the amplitude of the scala media potential recorded at the BP. It should be noted that even though Fig. 5.4 shows that the amplitudes of the $\mathrm{CM}$ are changed by the longitudinal coupling, this does not mean that the broadness of the CM tuning changes. However these amplitude changes may be important to determine whether there are missing OHCs along the cochlear partition similar to the modelling approach advocated by (Chertoff et al., 2012). These amplitude changes may have viable clinical applications (see Section 7.3).

For making a quantitative judgment, bandwidth $(3 \mathrm{~dB})$ is also computed using the relationship between frequency and spatial tuning curves (Geisler and Cai, 1996) 


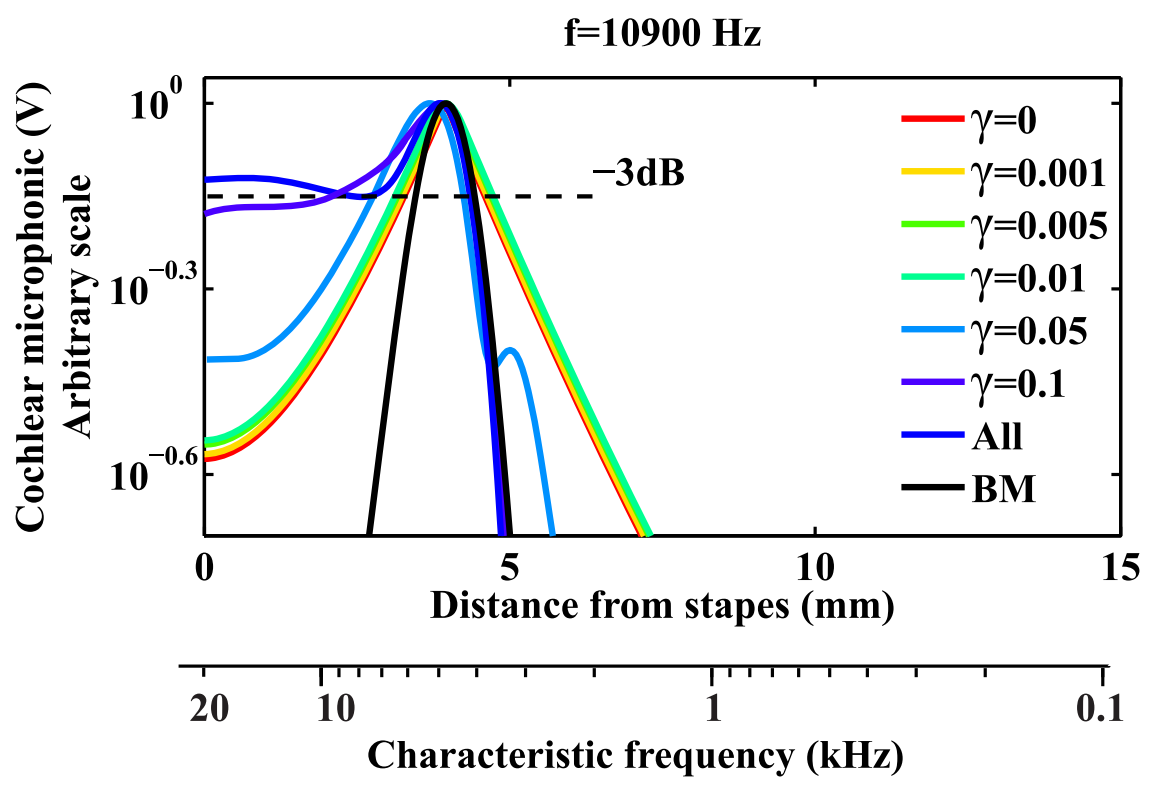

Figure 5.3: The CM spatial tuning curves for stimulus frequency of $10900 \mathrm{~Hz} . \gamma$ is the proportion of the cochlea that is electrically active on each side of the best place.

and using Greenwood's function (Greenwood, 1990a). The results are shown in Fig. 5.5. These qualitative evaluations also suggest that longitudinal coupling does not considerably increase the bandwidth.

\subsection{Effect of Transversal Phase Cancellation on the CM Tuning Curves}

To inspect the effect of transversal phase cancellation, the amplitudes of $\mathrm{OHC}$ potential variables are shown as a function of the cochlear position in Fig. 5.7. Despite the very similar amplitudes of $V_{\mathrm{OHC}}$ and $V_{\mathrm{HB}}$, they have a phase difference of $\pi$ near their peaks and nearly cancel each other out (see Fig. 5.8). 


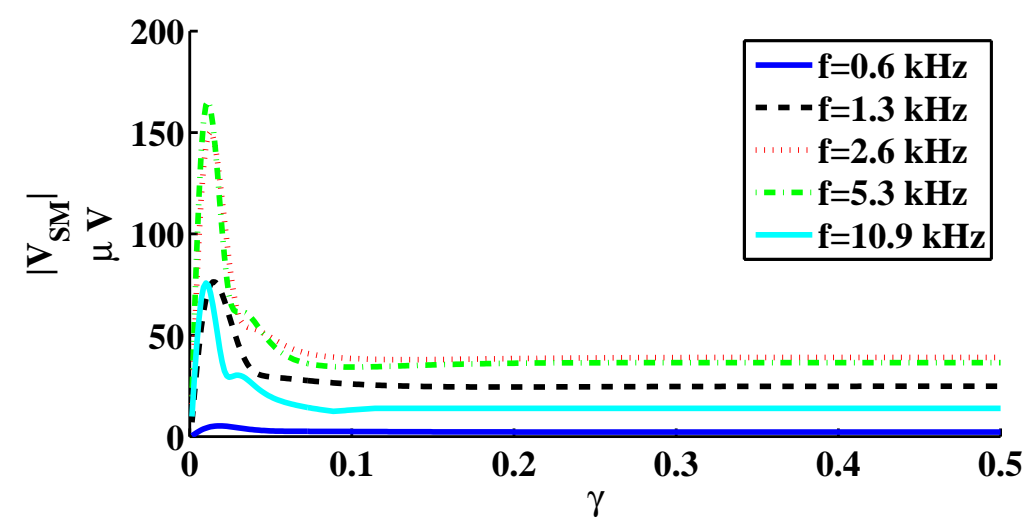

Figure 5.4: Amplitude of the scala media potential at 23.8, 19.4, 14.6, 9.7 and $4.6 \mathrm{~mm}$ from the stapes and stimulus frequencies $0.6,1.3,2.6,5.3$ and $10.9 \mathrm{kHz}$ respectively. Abscissa is $\gamma$ the proportion of the cochlea that is electrically active on each side of the best place.

\subsubsection{Longitudinal Resistors}

For all simulations here, each section is $50 \mu \mathrm{m}$ in length, which accounts for 5 OHCs in each row (15 OHCs) assuming that the human cochlea contains 3500 OHCs in each row (10500 OHCs) (Ashmore, 2008). A longitudinal space constant of $70 \mu \mathrm{m}$ for the human cochlea ${ }^{2}$ is adequate to reproduce realistic responses ( $\mathrm{Ku}$, 2008).

The adjacent regions of the organ of corti connect to each other via the fluids inside the scala vestibuli, the scala media and scala tympani which are modelled as longitudinal resistors $R_{8}, R_{9}$ and $R_{11}$, respectively (Fig. 3.7). Their parameter values were derived by using specific resistances of perilymph and endolymph (Misrahy et al., 1958; Tran et al., 2013) and the cross-sectional area of scalae in humans (Wysocki, 1999; Thorne et al., 1999) in Appendix C.

Changing these resistor values causes different potential coupling effects. To investigate how these values affect the $\mathrm{CM}$, they are scaled by scaling factor $\zeta$. We noticed that scaling these values to less than the modified values of Strelioff (1973)

\footnotetext{
${ }^{2}$ The average length of the human cochlea is $35 \mathrm{~mm}$, and $70 \mu \mathrm{m}$ require 500 segments.
} 
(a)

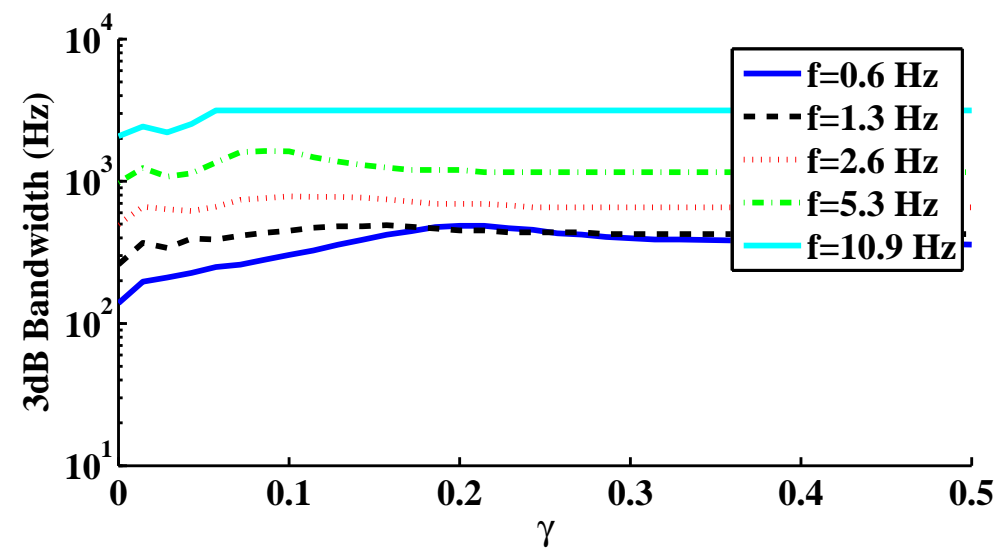

(b)

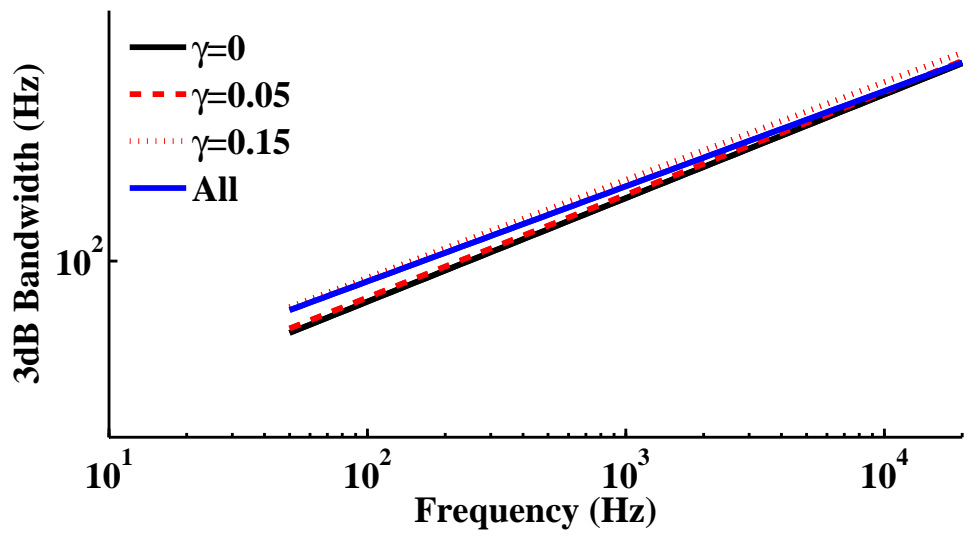

Figure 5.5: (a) shows $3 \mathrm{~dB}$ bandwidth of the $\mathrm{CM}$ tuning curves for different frequencies. Abscissa is $\gamma$ (the proportion of electrically active section). (b) shows $3 \mathrm{~dB}$ bandwidth for the human cochlea versus frequency. These results show that the broadness of the $\mathrm{CM}$ is not changed significantly by active longitudinal coupling.

for humans deteriorates amplification ability of the model cochlear amplifier and scaling by 0.1 dramatically changes the currents in each section, and therefore gravely reduces the amplification ability of the model cochlear amplifier. The model mechanical behaviours are not significantly changed by increasing longi- 


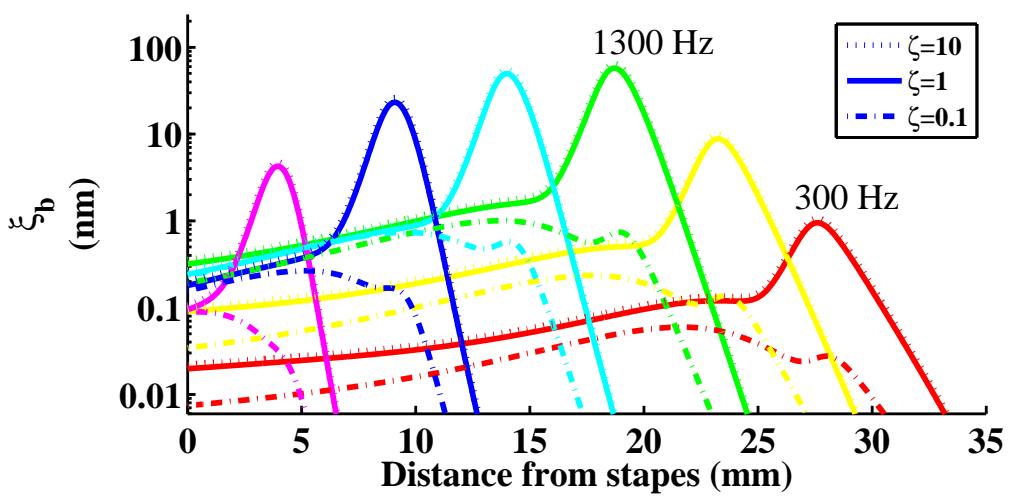

Figure 5.6: The magnitude of the basilar membrane displacement, as a function of cochlear position for six different stimulus frequencies: 10900, 5300, 2600, 1300, 600 and $300 \mathrm{~Hz}$. $\zeta$ is the longitudinal resistor scaling factor. In the proposed model, increasing the longitudinal resistance values does not change the magnitude of the basilar membrane displacement noticeably. Decreasing these values however, does change the magnitude of the basilar membrane displacement and deteriorates the amplification ability of the model.

tudinal resistor values. Fig. 5.6 compares the magnitude of the BM displacement for three longitudinal resistors scaling factors: $0.1,1$ and 10 .

Scaling the values of the longitudinal resistors more than 1 (e.g., 10) increases the sharpness of the $\mathrm{CM}$, and when their values become very large (infinite i.e., open circuit) the CM becomes as sharply tuned as the BM motion curves. The results indicate that longitudinal coupling affects the phase difference between $V_{\mathrm{HB}}$ and $V_{\mathrm{OHC}}$. Fig. 5.8 (b) shows the effect of increasing the longitudinal resistances. When these resistances are close to physiologically realistic values, the phase difference near the best place is approximately $\pi$ and the summation result of $V_{\mathrm{HB}}$ and $V_{\mathrm{OHC}}$ (i.e., the $\mathrm{CM}$ ) is attenuated. For more apical locations this phase difference approaches zero and the summation results of these two potentials are not attenuated. A combined effect of cancellation near the peak area and summation elsewhere causes the CM broad tuning curves. 
(a)

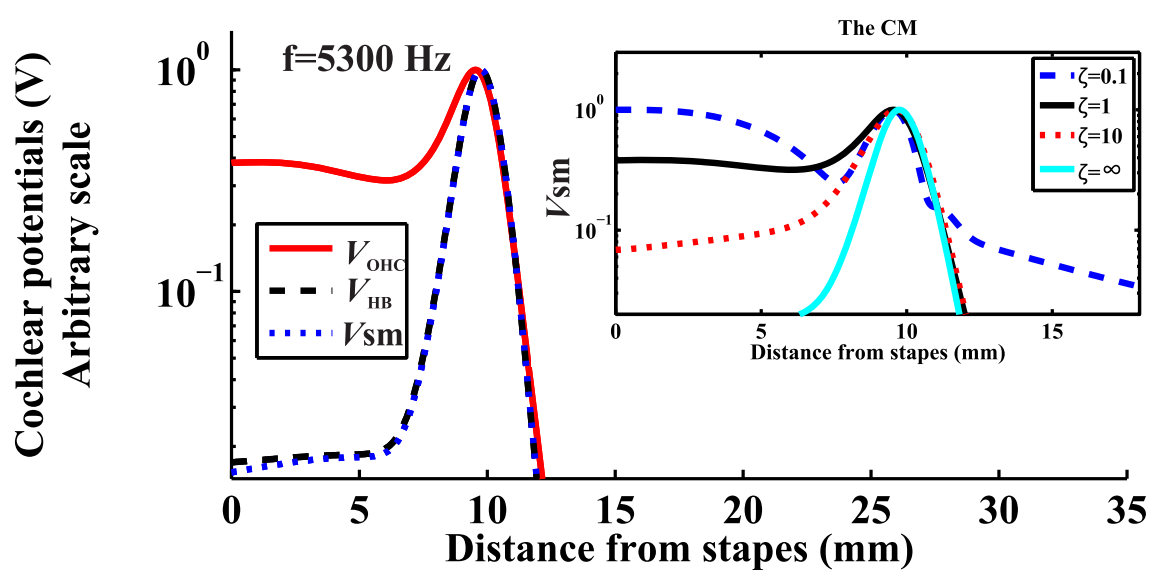

(b)

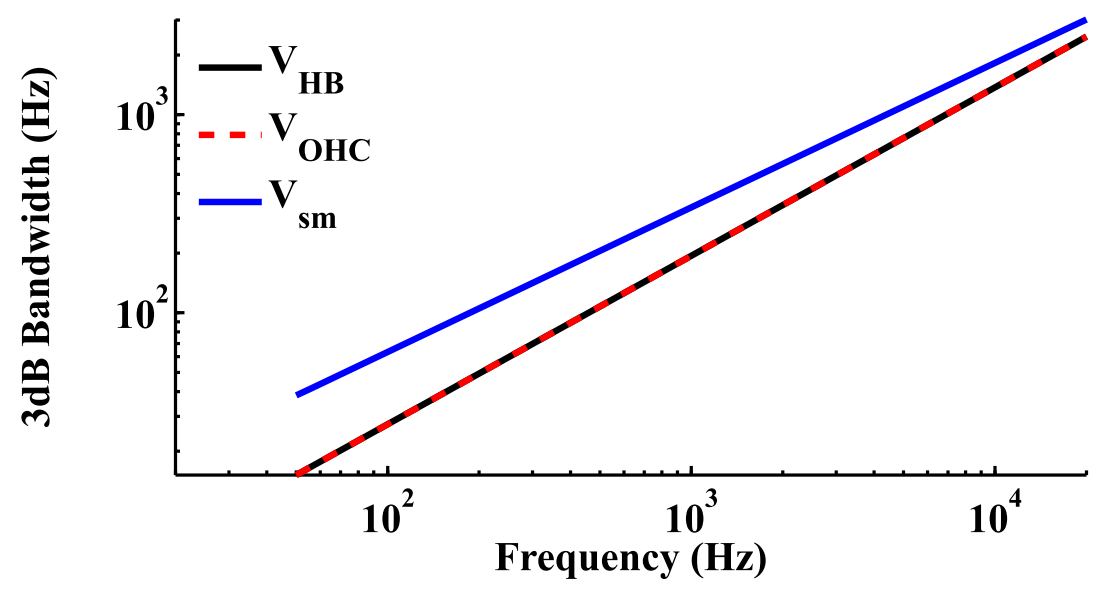

Figure 5.7: (a) Amplitudes of $V_{\mathrm{OHC}}, V_{\mathrm{HB}}$ and $V_{\mathrm{sm}}$ for stimulus frequency of $5300 \mathrm{~Hz}$. All curves are normalised to their maximum values. As can be seen from panel (a), the amplitude of $V_{\mathrm{sm}}$ (the $\mathrm{CM}$ ) is much broader than $V_{\mathrm{OHC}}, V_{\mathrm{HB}}$. The part of this panel labelled "The CM" shows how the scaling of the longitudinal resistor values changes the $\mathrm{CM}$. $\zeta$ is the longitudinal resistors scaling factor. (b) shows $3 \mathrm{~dB}$ bandwidth difference for the audio frequency range. 
(a)

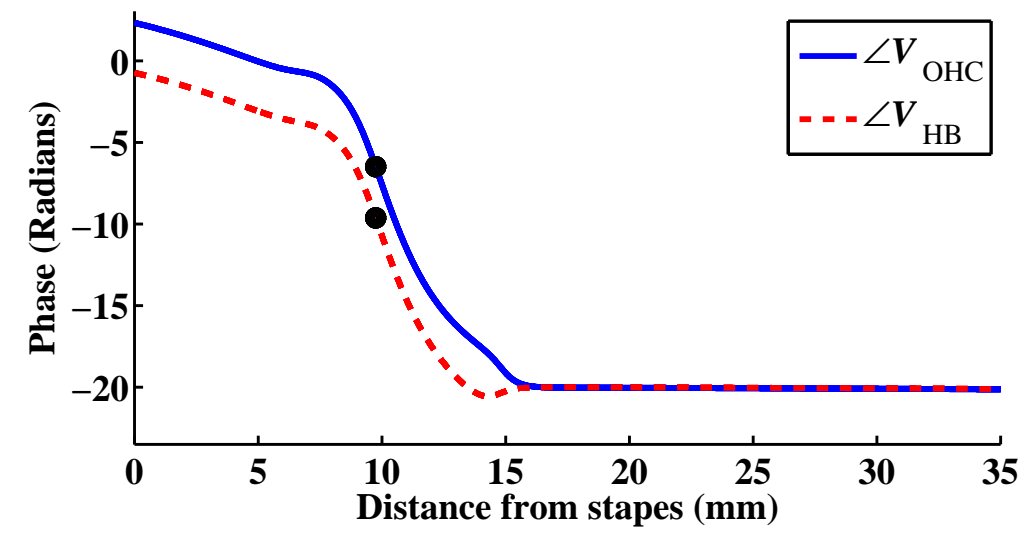

(b)

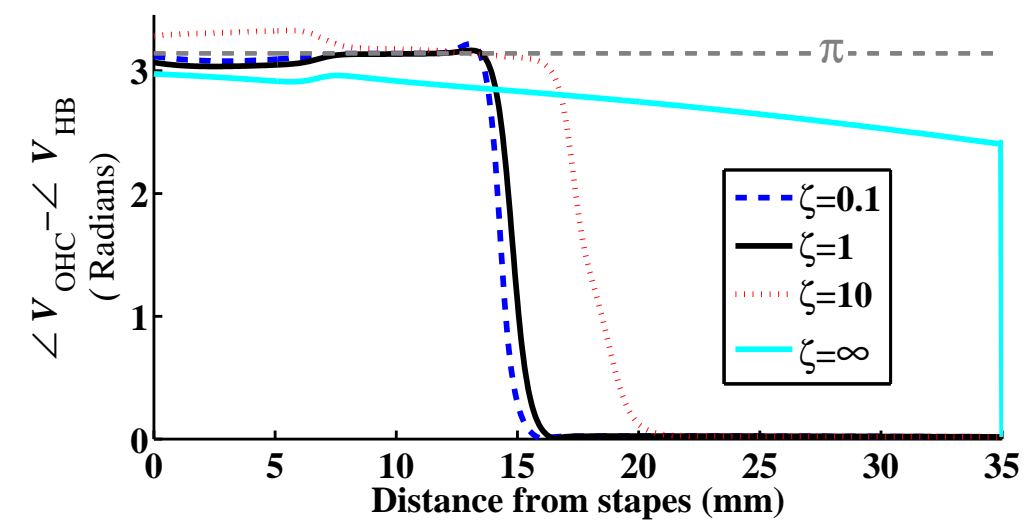

Figure 5.8: (a) Phase of $V_{\mathrm{OHC}}$ and $V_{\mathrm{HB}}$. (b) Phase difference of $V_{\mathrm{OHC}}$ and $V_{\mathrm{HB}}\left(\angle V_{\mathrm{OHC}}-\angle V_{\mathrm{HB}}\right)$. For clarity, only curves for the characteristic frequency of $5300 \mathrm{~Hz}$ for different longitudinal resistors scaling (see Section 5.3.1) are shown. $\zeta$ is the longitudinal resistors scaling factor.

\subsubsection{Discussion}

Changing the longitudinal resistors of the model alters the sharpness of the CM. In fact passive longitudinal coupling (i.e., coupling without active $\mathrm{OHCs}$ ) affects the transversal phase difference. Fig. 5.2 and 5.3 indicate that the CM measured at the best place (when only the section of the best place contributes) still exhibits broad tuning. 
From all these findings it can be concluded that the difference in sharpness between the tuning curves of basilar membrane motion and the $\mathrm{CM}$ is mainly because of the phase cancellation between the hair bundle potential and the potential of the body of the $\mathrm{OHC}$ rather than longitudinal phase cancellation along the cochlea.

The conclusion proposed here agrees with the sharp tuning exhibited in intracellular measurements of the OHC (OHC soma) reported in Russell (2008). The conclusion can be more specifically verified or denied by simultaneous measurement of $\mathrm{OHC}$ soma potential and both scala media and tympani potentials to determine the phase difference between $V_{\mathrm{OHC}}$ and $V_{\mathrm{HB}}$. To the best of our knowledge, this measurement has not yet been reported.

\subsection{Summary}

Even though the broad tuning curves of the CM generated by the PCM agree with experiments, these results are surprising considering the sharp tuning of the $\mathrm{BM}$ displacement. Researchers and modellers have postulated that the $\mathrm{CM}$ in each recording place is a vectorial average of active OHCs in different locations along the cochlea and the vectorial average produces this discrepancy.

We use the proposed electromechanical model to examine the effect of longitudinal coupling on the broadness of the CM tuning curves. This effect is assessed both objectively and visually. The results of the model demonstrate that active longitudinal coupling (i.e. coupling with active $\mathrm{OHCs}$ ) contributes only slightly to the broadness of the CM tuning curves (Fig. 5.2 and 5.3). Another factor must therefore be involved.

By inspecting the OHCs potentials, a phase difference between $V_{\mathrm{OHC}}$ and $V_{\mathrm{HB}}$ of approximately $\pi$ can be seen. Therefore, the sum of these two potential results in a broader potential (the $\mathrm{CM}$ ). This is a significant contributing factor to the broadness of the CM tuning curves based on the proposed approach. 
To summarise, the proposed approach showed that longitude passive network of the organ of Corti affects the phase difference between $V_{\mathrm{OHC}}$ and $V_{\mathrm{HB}}$ and causes transversal phase cancellation in radial sections of the organ of Corti near the best place and results in broader tuning of the $\mathrm{CM}$.

It is noteworthy that even though the vectorial average changes the amplitude of the CM (Fig. 5.4), it does not mean it changes the broadness as well. The results demonstrate that the passive network of the organ of corti has the main influence on the broadness of the $\mathrm{CM}$ and the transversal cancellation broadens the tuning curve of the CM.

\subsection{Contribution}

- The effect of longitudinal coupling versus transversal phase cancellation on the broadness of the CM tuning curves based on the proposed model was assessed.

- The proposed approach hypothesises that the broadness of the CM tuning curves is mainly due to transversal cancellation rather than longitudinal cancellation. 


\section{Chapter 6}

\section{Spontaneous Cochlear Microphonic}

\subsection{Introduction}

Cochlear models have been extensively studied and developed to help enrich our understanding about the physical and biological mechanisms engaged in the hearing process (Chadwick, 1997). Cochlear models are developed based on known cochlear structures and functions. Therefore, a cochlear model that can reproduce realistic cochlear responses, may make predictions about as yet unstudied phenomena and stimulate further physiological experiments (Wen, 2006). In this chapter, we discuss the possibility of a hitherto unreported phenomenon; spontaneous cochlear microphonic.

As previously discussed in Section 2.3, SOAEs are one the major classes of OAEs, and can be detected in the ear canal without any acoustic stimulus. Existence of these emissions is an explicit manifestation of the active mechanism in the cochlea (Probst et al., 1991; Vilfan and Duke, 2008).

There is evidence that self-sustaining oscillation of the BM in the cochlea can cause vibration of the ear drum and produce SOAEs (Robles and Ruggero, 2001). Preexisting mechanical perturbations which are hypothesised to be the origin of SOAE (Shera and Guinan, 2008) can be considered as spatial inhomogeneities 
in the cochlea structure $(\mathrm{Ku}, 2008)$. However the exact mechanisms involved in generating SOAEs are as yet undiscovered. There are two different theories that have been established to explore the origin of SOAE.

The global standing-wave model proposes that SOAEs are produced by coherent reflections between an impedance mismatch at the middle ear and perturbations in the mechanics of the cochlea. The SOAE amplitude is maintained and stabilised by the cochlear amplifier (Shera, 2003). The second model, known as the local oscillator model, suggests that the active elements inside the cochlea independently cause local oscillation (Braun, 2013).

In the global standing-wave model, the spontaneous frequencies that can be generated exhibit a minimum frequency spacing (or preferred minimum distance (PMD)) as Shera and Guinan (2008) report. In other words, multiple coherent internal reflections from multiple irregularity sites form a standing wave and hence produce multiple SOAEs with a certain frequency spacing (Shera, 2003). The local oscillator model predicts no constraint on the spontaneous frequencies.

From a modelling point of view, abrupt changes in the cochlear amplifier gain of the human cochlear model can cause oscillation in the basilar membrane that can be detected in the ear canal as SOAEs (Nobili et al., 2003; Elliott et al., 2007; Rapson et al., 2012).

The proposed model analogously shows that these perturbations can also produce Spontaneous COchlear MICrophonic (SCOMIC). The work which is presented in this chapter is implementing the approach of Elliott et al. (2007); Ku (2008) on the proposed model and the extension is to infer the existence of the SCOMIC. In addition, the simulations show that our model is more similar to the local oscillator model and does not necessarily include the minimum frequency spacing.

This chapter begins with assessing the stability of the proposed model in Section 6.2. The effect of irregularities on the stability is discussed in Section 6.2.1. Finally time-domain simulations and the existence of the SCOMIC are presented in Section 6.2.2. 


\subsection{Stability of the Proposed Model}

One definition of system stability is that a system is considered to be stable if every bounded input produces a bounded output (Dorf and Bishop, 2011). To evaluate stability of a linear system the transfer function of that system should be examined. The transfer function of a linear system is defined as the ratio of the Laplace transform of the output to the Laplace transform of the input, assuming that all initial values are zero. By taking the Laplace transform of equation (4.2), we have:

$$
\mathbf{X}(s)=\left((s \mathbf{I}-\mathbf{A})^{-1} \mathbf{B}\right) \mathbf{U}(s)
$$

where $\mathbf{X}(s)$ and $\mathbf{U}(s)$ are Laplace transforms of $x(t)$ and $u(t)$ respectively, and I is the identity matrix. This relation is the transform function of the model. Note that the roots of the determinant of $s \mathbf{I}-\mathbf{A}$ are the poles of the system. This determinant is called the characteristic polynomial and the roots of the characteristic polynomial are the eigenvalues $(\lambda)$ of $\mathbf{A}$ (Dorf and Bishop, 2011).

Each system pole corresponds to a time-domain term, which can be written as $e^{\sigma t} \cos (\omega t)$, where $\sigma$ is the real part of $\lambda$ and $\omega$ is its imaginary part $(\lambda=\sigma+j \omega)$. $\sigma$ can be observed as the decay or growth rate by which the time domain response of the model, associated to this term, decays to zero or grows to infinity as time goes to infinity. $\omega$ determines the oscillation frequency of this term.

Therefore, to have a stable system, all poles of the transfer function should have negative real part (i.e., $\Re(\lambda)<0$ ).

To assess the stability of the proposed model, the poles of the system or the eigenvalues of $\mathbf{A}$ are calculated and shown in Fig. 6.1. It is worth mentioning again that the matrix $\mathbf{A}$ is calculated by linearizing the mechanoelectrical transduction (MET) current $i_{\mathrm{r}}$ (refer to Sections 3.4.1 and 4.1). All coefficients of the model are real, hence the roots are located symmetrically with respect to the real (horizontal) axis. Henceforth, for the sake of clarity and without losing generality, the roots with positive imaginary values are only shown. Fig. 6.1 shows that the model with original parameter values does not have any poles with negative real part and 
(a)

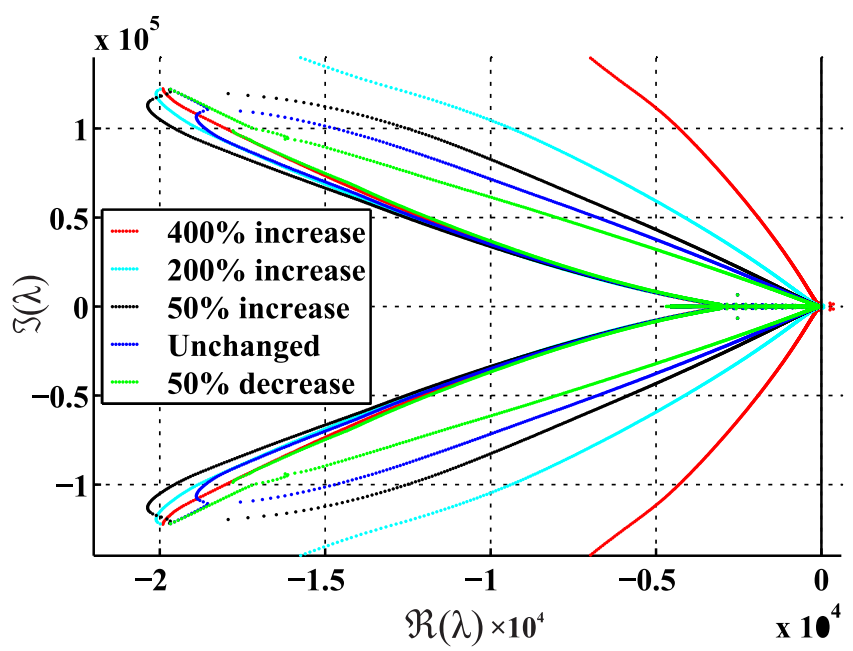

(b)

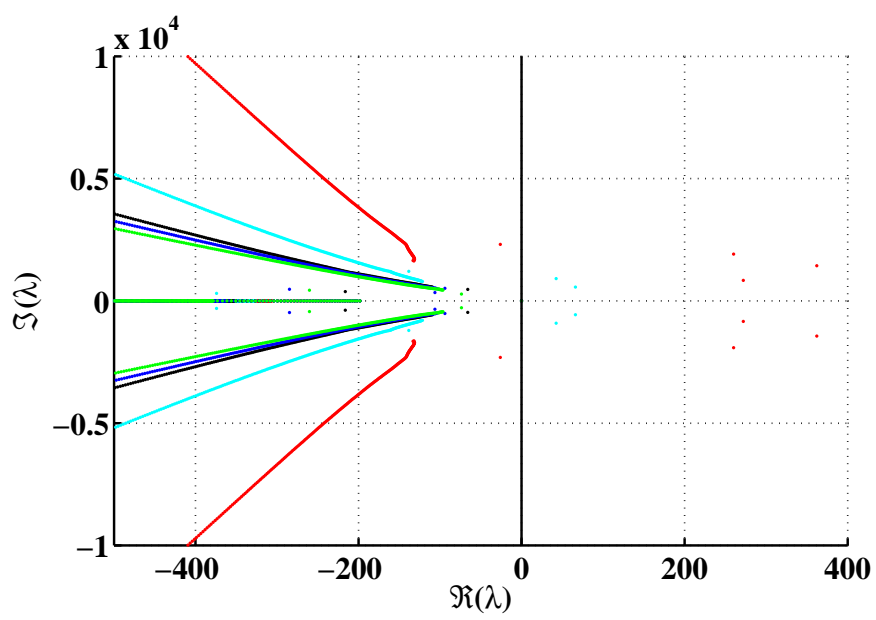

Figure 6.1: Pole location of the proposed model. The abscissa is the real part of the poles $(\Re(s))$. The ordinate is the imaginary part of the poles $\left(\mathfrak{I}(s)\right.$ ). 50\% increase of both $\alpha_{\mathrm{v}}$ and $\alpha_{\mathrm{d}}$ (the MET's sensitivity to reticular lamina velocity and displacement) does not make the system unstable. 200\% increase of both $\alpha_{\mathrm{v}}$ and $\alpha_{\mathrm{d}}$ is required for archiving instability. Zoomed around the origin of (a) is shown in (b). Note that poles are either purely real, or appear in complex conjugate pairs which make the pole location symmetric with respect to the real axis. 
therefore it is stable. Computer simulations of the proposed model indicate that increasing or decreasing values of the velocity and displacement to current gain $\left(\alpha_{\mathrm{v}}\right.$ and $\alpha_{\mathrm{d}}$ ) of the proposed model substantially (for example by 50\%) affect the mechanical and electrical outputs of the model. However, the simulations also demonstrate the important observation that these changes do not make the system unstable. To achieve instability an increase of approximately $200 \%$ is required. This is a difference between the proposed model and the model of Elliott et al. (2007); $\mathrm{Ku}$ (2008). The proximity of the contour of poles to the imaginary axis can be controlled by the feedback gain in the model of Elliott et al. (2007); Ku (2008). When a contour of poles in the model of Elliott et al. (2007); Ku (2008) is near the imaginary axis (i.e. poles with small negative real part) (Fig. 7. of Elliott et al. (2007)) and the model is close to instability, small changes in the positive feedback gain of their model shift these poles to the right hand side of the imaginary axis and makes it unstable. In contrast, changing the parameters does not bring the PCM close to instability.

\subsubsection{Effects of Irregularities on the Stability}

Investigations on chinchilla ears have demonstrated that SOAEs can occur after noise exposure. Histopathological examination of the emitting cochlea indicated that the SOAEs frequencies correspond to the damage positions on the basilar membrane (Clark et al., 1984). Studies have also shown that experimental animals (especially macaque monkeys which have more easily detectable SOAEs than other nonhuman primates) have irregularities in the arrangement of $\mathrm{OHCs}$ at some places on the BM associated with the pertinent frequencies of the detectable SOAEs (Lonsbury-Martin and Martin, 1988). By comparing these two studies, some researchers have postulated that both imposed and natural SOAEs are results of the same mechanism. The former is due to imposed imperfection and the latter is because of natural imperfection in the cochlea (Bright, 2007). It should be noted that SOAEs can be detected in ears without noticeable histopathologic changes, and mechanisms involved in generating SOAEs may vary between species (Probst 
et al., 1991).

Fig. 6.1 illustrates that the model is robust to scaling of parameter values over the entire length of the cochlea. However, even small but abrupt spatial inhomogeneities in the cochlear amplifier gain of the model can make the model unstable (Nobili et al., 2003; Elliott et al., 2007; Rapson et al., 2012),.

(a)

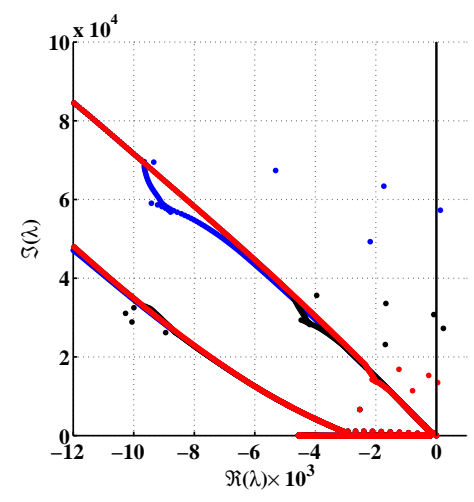

(c)
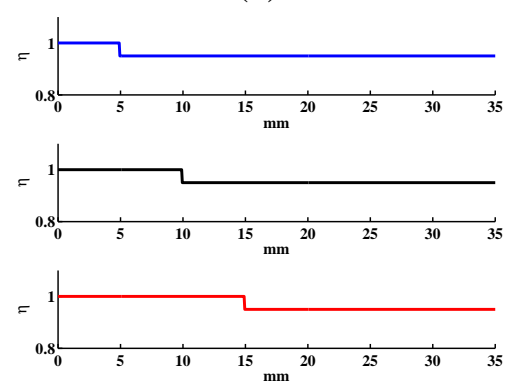

(b)

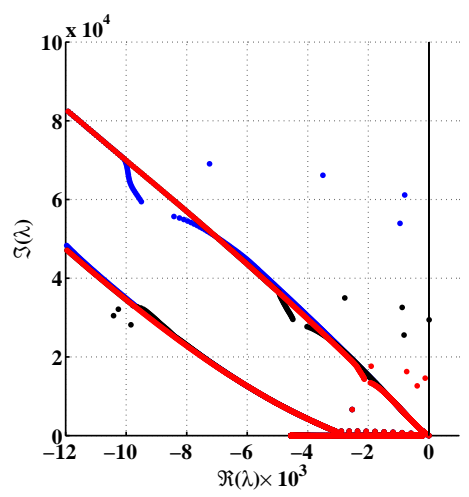

(d)
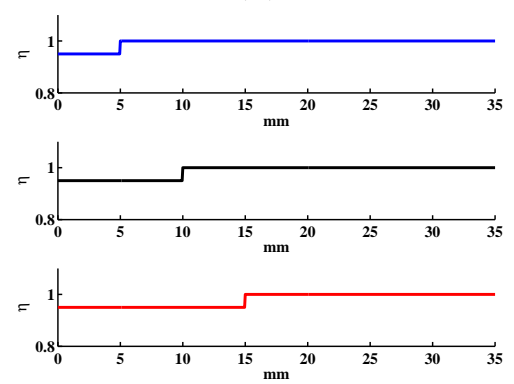

Figure 6.2: The effect of a step change on the stability of the model. (a) shows the pole location for the distributed control parameter coefficient defined as $\eta(x<=5,10,15 \mathrm{~mm})=1$ and $\eta(x>$ $5,10,15 \mathrm{~mm})=.95$ and (b) shows the pole location for the irregularity coefficient defined as $\eta(x<=5,10,15 \mathrm{~mm})=0.95$ and $\eta(x>5,10,15 \mathrm{~mm})=1$, (c) and (d) show the irregularity coefficients of panels (a) and (b), respectively.

The profile of the spatial inhomogeneities in the human cochlea is as yet unexplored (Ku, 2008). Step change in the spatial inhomogeneities and random change of the spatial inhomogeneities are two plausible assumptions which are 
explored in the following. For this purpose, the distributed control parameter coefficient, $\eta$, is defined to vary the parameters of the model.

For investigating the effects of abrupt spatial inhomogeneities, $\alpha_{\mathrm{v}}$ is varied. It should be noted that varying other parameters such as $\alpha_{\mathrm{d}}$ was found to have similar effects on the stability of the model. Fig. 6.2 demonstrates $5 \%$ step changes in the

(a)

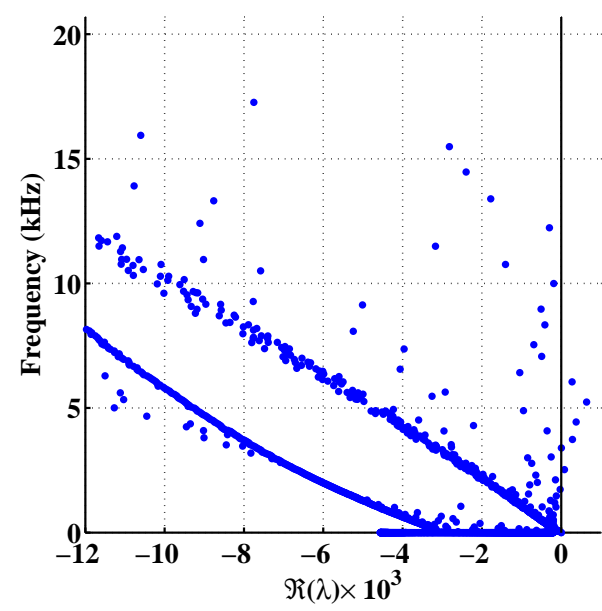

(c)

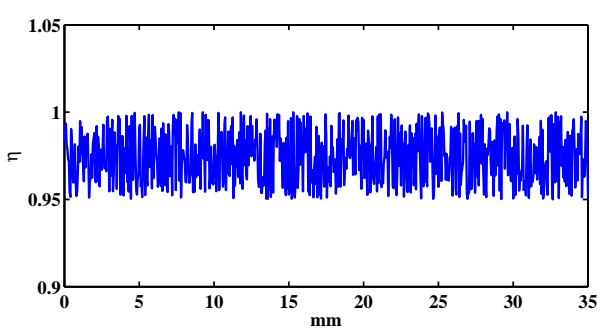

(b)

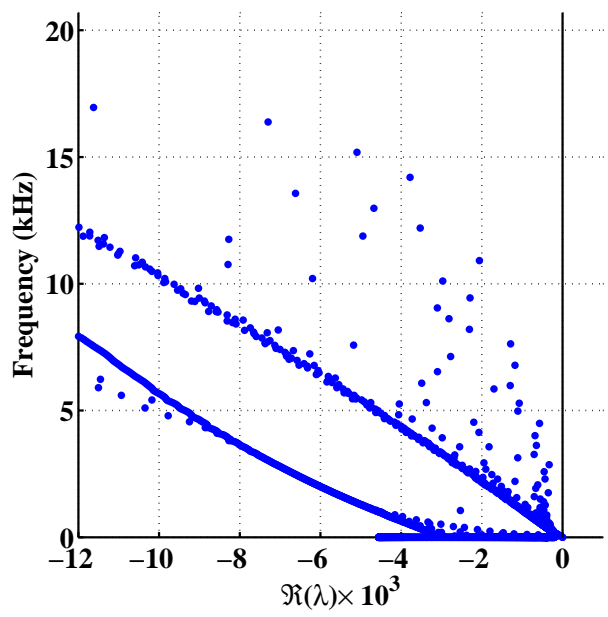

(d)

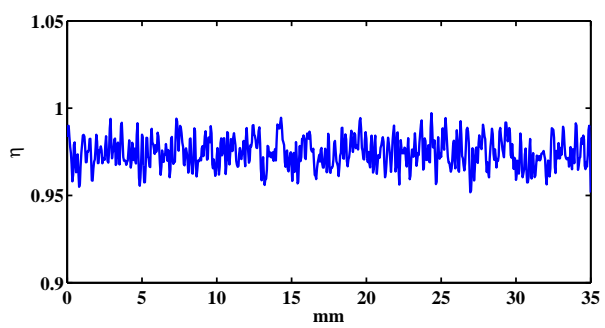

Figure 6.3: (a) Rapid inhomogeneities imposed on $\alpha_{\mathrm{v}}$ by the distributed control parameter coefficient $(\eta)$ make the system unstable. (b) Smooth varying of $\alpha_{\mathrm{v}}$ does not make the system unstable. $\eta$ is generated using uniformly distributed pseudorandom numbers on the interval $[0.95,1]$ panel (c) and smoothed using a 3 point moving average filter (d). The abscissa here is in $\mathrm{kHz}$ instead of radian/s.

distributed control parameter coefficient $\left(\eta=1\right.$ for $x<=x_{0}$ and $\eta=0.95$ for $\left.x>x_{0} \mathrm{~mm}\right)$ and $\left(\eta=0.95\right.$ for $x<=x_{0} \mathrm{~mm}$ and $\eta=1$ for $\left.x>x_{0}\right)$ at the locations 
$\left(x_{0}\right) 5,10$ and $15 \mathrm{~mm}$ from the stapes.

Fig. 6.3 illustrates the effects of random change in the distributed control parameter coefficient. Smooth random inhomogeneities do not destabilise the model (Fig. 6.3 (b)) while rapid inhomogeneities with the same amplitudes make the proposed model unstable (Fig. 6.3 (a)). In panel (c) of Fig. 6.3, $\eta$ is the uniform distribution on the interval $[0.95,1]$ which is smoothed in panel (d) using a 3 point moving average filter.

We now repeat previous experiments 500 times and obtain the histogram shown in Fig. 6.4. Comparing the results of panel (a) and (b) of Fig. 6.3 and Fig. 6.4 indicates that rapid inhomogeneities or discontinuities are very likely to make the model unstable.

As mentioned earlier, unlike the model of Elliott et al. (2007); Ku (2008), The PCM is not close to instability and perturbations of the parameters do not produce many unstable poles as the model of Elliott et al. (2007); Ku (2008) does (Figure 3.9. of $\mathrm{Ku}(2008))$.

\subsubsection{Time-domain Simulations of the Model Including Irregularities and the existence of SCOMIC}

The general interpretation of a pole in time-domain addressed in Section 6.2 is consistent with time-domain simulations presented in Chapter 4. When a click reaches the oval window, a travelling wave forms that travels from the base to the apex. At each region of the organ of Corti, the BM oscillates with the frequency $\omega$ of the pole associated with the region and decays away by the rate of $\sigma$ (Fig. 4.8). As examined and discussed thus far, the rapid spatial irregularities can generate poles with positive real parts (i.e., positive $\sigma$ s) and consequently make the model unstable.

For assessing the transient behaviours of the model including poles having positive real parts, time-domain analyses are performed to investigate the effect of step 
(a)

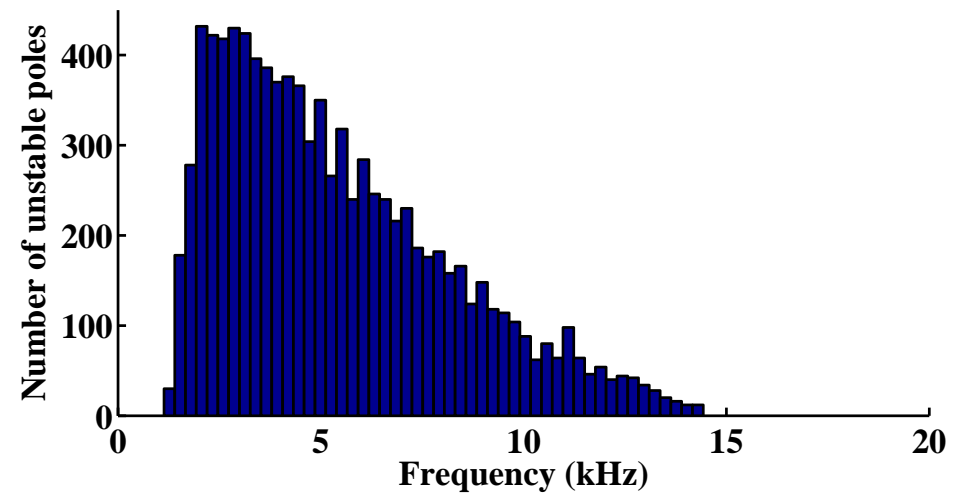

(b)

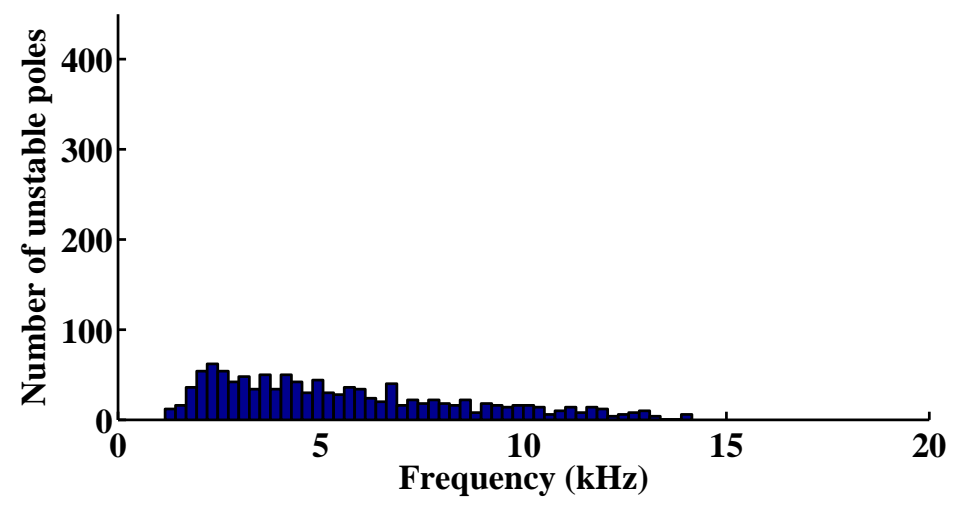

Figure 6.4: Histograms for cochlear models with randomly generated $\eta$. For the histogram in panel (a) $\eta$ is the uniform distribution on the interval $[0.95,1]$ which is smoothed for the histogram in panel (b) using a 3 point moving average filter. The experiments were repeated 500 to obtain the histograms.

changes in the distributed control parameter coefficient shown in Fig. 6.2.

The results of time-domain analysis for $\eta(x<=10 \mathrm{~mm})=0.95$ and $\eta(x>$ $10 \mathrm{~mm})=1$ are presented in Fig. 6.5. A $0 \mathrm{~dB}$ SPL click with duration $40 \mu \mathrm{s}$ is used as initial input that could correspond to ambient or physiological noise to initially elicit the spontaneous otoacoustic emissions.

Fig. 6.5 (blue solid line) shows that for the linearized form of the MET channel 
current $\left(i_{\mathrm{r}}\right)$ response, the BM displacement grows to infinity as expected from the time-domain term associated with the pole having positive real part. The MET channel current $\left(i_{\mathrm{r}}\right)$ response however, actually has a nonlinear saturation property (see equation (3.18)) which stabilises the amplitude of the BM oscillation (Fig. 6.5 (red dashed line)) and thus results in spontaneous self-sustaining oscillation of the BM. This kind of self-sustained oscillation is known as a limit cycle (Khoo, 2000), and can be considered the source of SOAEs (Elliott et al., 2007; Ku et al., 2009). The numerically observed finite amplitude behaviour of the BM oscillation is consistent with Moreno and Suárez (2004) observation that saturation can lead to sable stationary points or orbits.

In general there is no systematic way to prove the existence of the stable limit cycle of a system of dimension higher than two and determine its amplitude (Hu et al., 2009). Some special cases are discussed in (Khalil, 2001, Chapter 5), Moreno and Suárez (2004); Bellizzi and Bouc (2007). Treating this topic from an algebraic point of view has its own extended domain of research and literature, and it is beyond the scope of this thesis to analytically examine the nonlinear stability of the system.

It is noteworthy however to mention here that with a random distribution of the distributed control parameter coefficient (Fig. 6.3), the model is more likely to have poles with positive real part for the characteristic frequencies less than $7 \mathrm{kHz}$ (Fig. 6.3 (a)). In other words, the model stability is more sensitive to rapid change near the apical end. This may be one of the factors influencing the predominance of SOAE between $500 \mathrm{~Hz}$ and $7000 \mathrm{~Hz}$ (Hall, 2000, Chapter 3). The forward and reverse middle ear gain characteristics may also influence this distribution (Puria, 2003). Preexisting mechanical perturbations cause self-sustaining oscillation in the BM which can be detected in the ear canal as SOAEs. Analogously these perturbations also produce spontaneous potentials in the cochlea as shown in Fig. 6.6. If the spontaneous cochlear microphonics predicted by the model do indeed exist they could be recorded in the generation sites. (For example at $10 \mathrm{~mm}$ inside the scala media) given appropriate experimental conditions. Fig. 6.7 (a)-(d) show the propagation of the displacement wave of the BM triggered by a click ( 60 
(a)

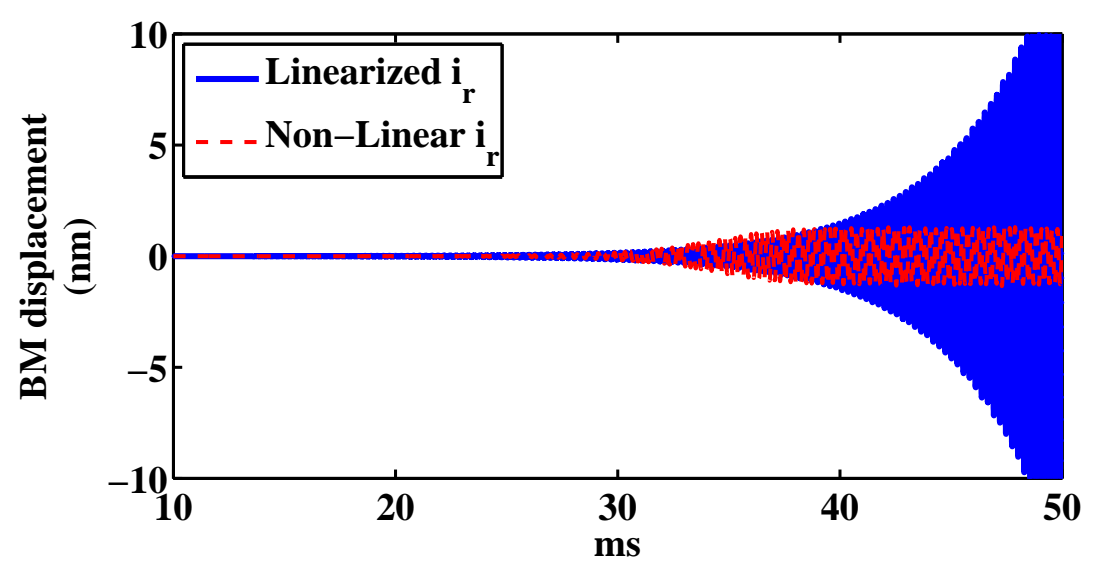

(b)

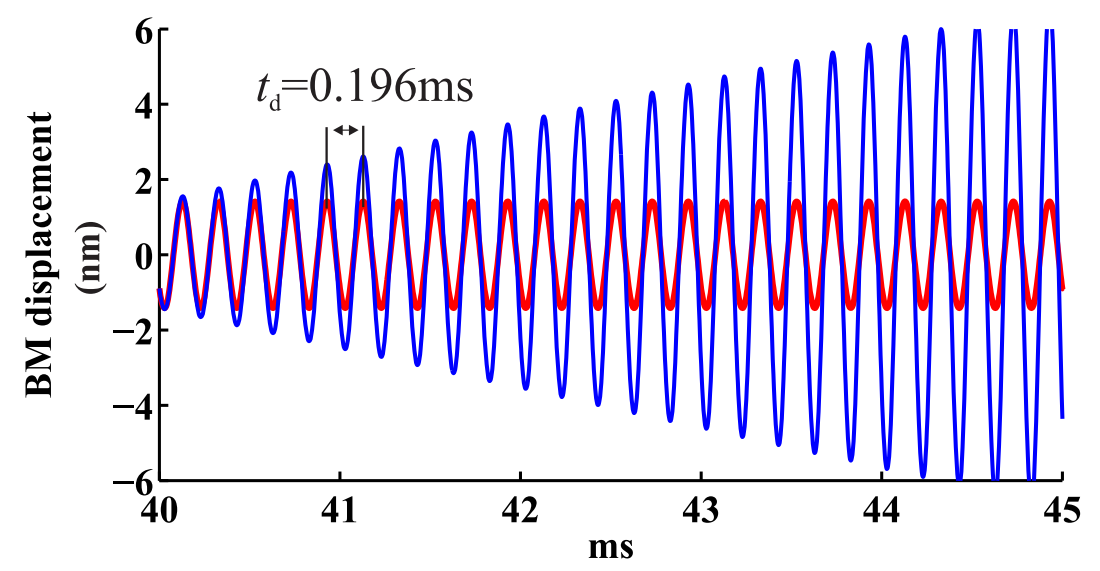

Figure 6.5: (a) shows the BM displacement with $\eta(x<=10 \mathrm{~mm})=0.95$ and $\eta(x>10 \mathrm{~mm})=1$ with the linear (blue solid line) and nonlinear (red dashed line) MET channel currents $\left(i_{\mathrm{r}}\right)(\mathrm{b})$ is zoomed-in version of panel (a). The saturating nonlinearity of $i_{\mathrm{r}}$ prevents unbounded growth of the BM displacement. The pole having positive real part produces a sine wave with $f \simeq 5100 \mathrm{~Hz}$ associated with abrupt spacial change at $10 \mathrm{~mm}$ based on the place-frequency map for humans.

$\mathrm{dB}$ SPL of duration $40 \mu \mathrm{s}$ ) along the cochlea for $\mathrm{t}=0.2, \mathrm{t}=1, \mathrm{t}=5$ millisecond and $\mathrm{t}=1$ minute, respectively. The ordinate is the basilar membrane displacement. (e)-(h) show the propagation of the CM wave along the cochlea. The ordinate is the CM amplitude. Note that a $0 \mathrm{~dB}$ SPL click as shown in Fig. 6.5 produces self- 
(a)

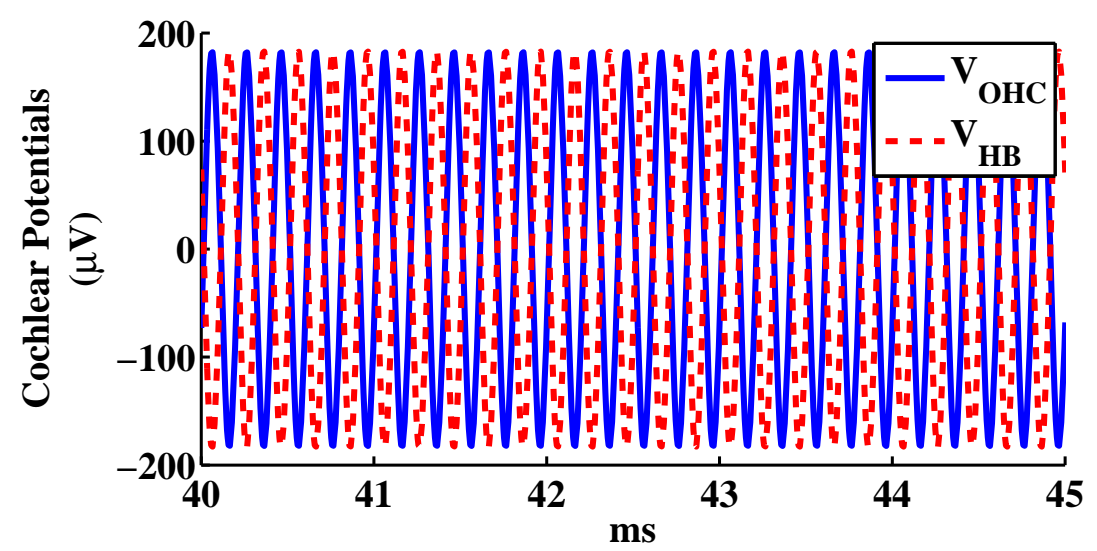

(b)

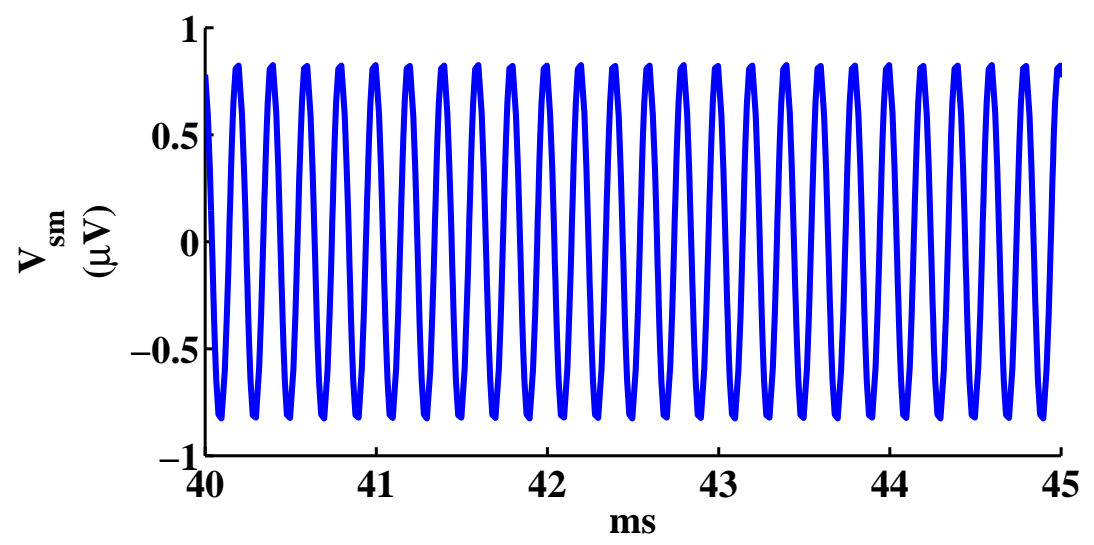

Figure 6.6: (a) shows spontaneous $V_{\mathrm{OHC}}$ (blue solid line) and $V_{\mathrm{OHC}}$ (red dashed line) with nonlinear (red dashed line) MET channel currents. (b) shows spontaneous $V_{\mathrm{sm}}$ (cochlear microphonic. The abrupt spacial change at $10 \mathrm{~mm}$ from the base produces the spontaneous potentials in the cochlea. These spontaneous potentials can be recorded at their generation site (here at $10 \mathrm{~mm}$ from the base).

sustained oscillations but the amplitude of the propagating wave is much smaller than the self-sustained oscillation. A larger ( $60 \mathrm{~dB}$ SPL) click is used in Fig. 6.7 to show the propagating wave as well. For experimental animals such as guinea pigs (Dallos et al., 2005; Honrubia and Ward, 1968) and mongolian gerbils (He and 
Ren, 2013), the CM has been measured at different locations along the cochlea. However recording the $\mathrm{CM}$ or other cochlear electrical responses near the base are more convenient and does not interfere with the cochlear function.

The proposed model is calibrated using the available data for human (see Appendix C). The actual SCOMIC amplitude based on the current model parameter values are between $0.01 \mu \mathrm{V}$ and $10 \mu \mathrm{V}$ for the human frequency range, and ought to be measurable with appropriate instrumentation. Experimentalists may also be able to record this signal at the round window (for higher frequencies which have generation sites near the base).

\subsection{Summary and Conclusion}

Since their discovery, SOAEs have been extensively studied, and recorded and have consequently been used to gain a good insight towards understanding the hearing mechanisms in mammals (Probst et al., 1991; Hall, 2000, Chapter 3).. Two theories have been hypothesised for SOAE generation; a) the global standingwave theory involves the existence of standing waves between the perturbation and the oval window for which the round trip delay is an integral number of cycles, and b) the local oscillator theory proposes that due to perturbations, the cochlear amplifier produces local oscillators without any coherent reflections being necessary (Shera, 2003; Braun, 2013).

Simulations using the proposed model show that small rapid perturbations in feedback gain can make the linearized cochlear model unstable. The saturation property of the MET channel current stabilises the unbounded growth of the BM displacement. The model of Shera (2003) involves the existence of standing waves between the perturbation and the oval window whereas the proposed model does not. Our model does not necessarily include the minimum frequency spaces for SOAEs that Shera and Guinan (2008) report, but challenged by Braun (2013). In this chapter we showed that the small rapid perturbations in feedback gain which have been previously hypothesised to be the generation source of 
(a)

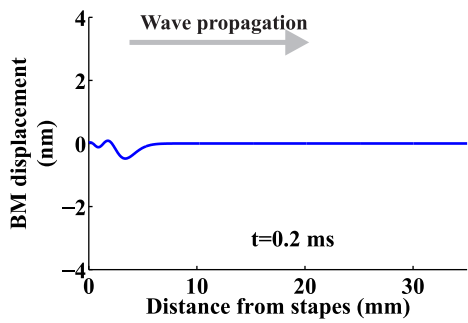

(b)

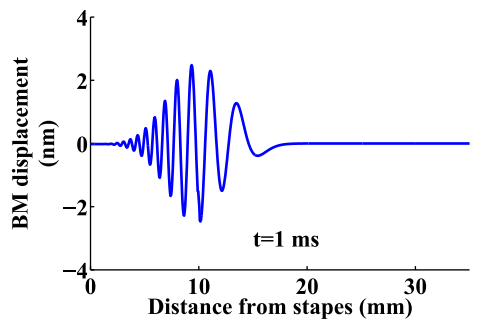

(c)

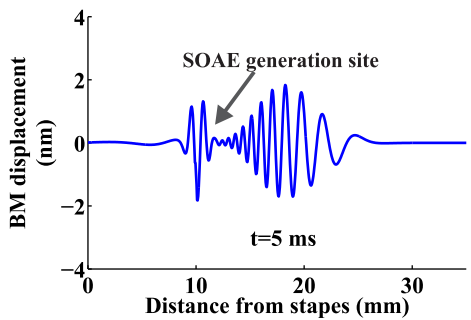

(d)

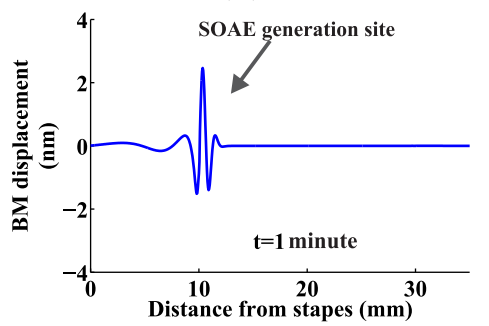

(e)

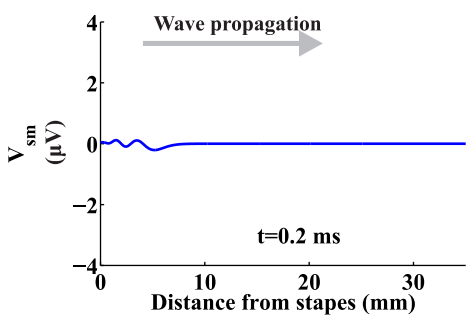

(f)

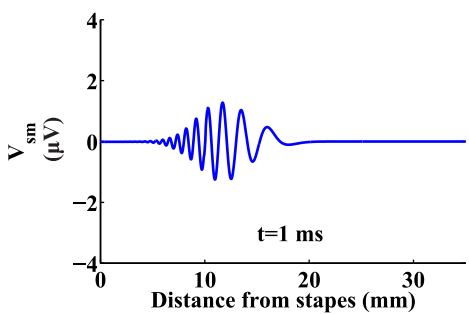

$(\mathrm{g})$

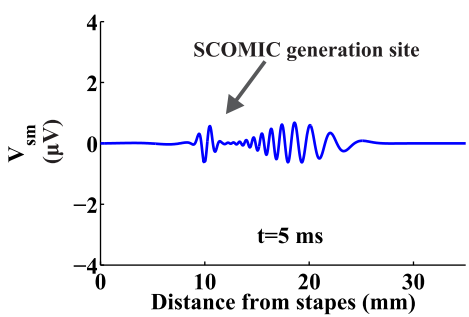

(h)

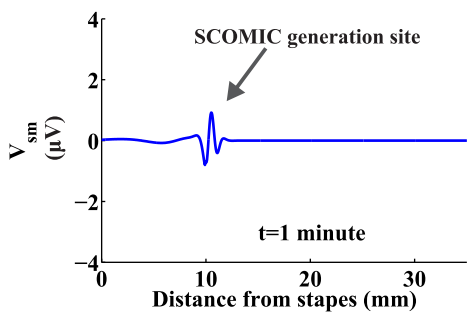

Figure 6.7: (a), (b), (c) and (d) show the model's predicted propagation along the cochlea of the BM displacement wave triggered by a click. Time domain analysis of the nonlinear cochlear model indicates that preexisting mechanical perturbations produce both SOAE, and Spontaneous Cochlear Microphonic (SCOMIC). 
SOAEs (Nobili et al., 2003; Elliott et al., 2007; Rapson et al., 2012) can also generate measurable Spontaneous Cochlear Microphonic (SCOMIC). Currently the SOAEs and SCOMIC generated by our model are localised to particular places along the BM associated with random irregularities. However, both theories of SOAE generation imply that the sources of the SOAEs are self-sustained oscillations along the basilar membrane which logically produce SCOMIC as well.

As explained so far, the SCOMICs are the electrical equivalents of the SOAEs and intuitively obvious. The question is why the existence of this signal has not yet been reported?

A possible reason could be that nobody has actually attempted to record this signal. Another reason could be that the amplitude of this signal is very small to measure. The spontaneous vibrations of the oval window are transmitted via middle ear and this facilitates in measuring SOAEs in the ear canal. In contrast, the SCOMIC is not transmitted, and so recording these electrical signals is more challenging. Our simulations based on human calibrated parameter values indicate that the amplitude of the SCOMIC can be in the range of one microvolt, similar to other bio-signals.

We have attempted to detect $\mathrm{CM}$ with the non-invasive measurement system of Masood et al. (2012) accompanying known SOAE (for human), but without success. At present we believe this is because of its small amplitude in noninvasive measurement rather than its absence. This signal may be detectable in human by a transtympanic recording system, or by electrodes inside the cochlea of animal accompanying known SOAEs.

\subsection{Contribution}

- The results of the proposed model indicate that small rapid perturbations in the feedback gain which have been previously hypothesised to be the 
generation source of SOAEs can also generate measurable Spontaneous Cochlear Microphonic (SCOMIC). 


\section{Chapter 7}

\section{Summary, Conclusions and Suggestions for Further Work}

Improvements in observation techniques for measuring mechanical vibration and electrical signals in the cochlea can increase our understanding of cochlear operation. However there still remain significant gaps in our understanding of the details of actual cochlear functions, which currently cannot be fully captured by contemporary instruments. In hearing studies, modelling techniques are employed to bridge these gaps and further the understanding of the cochlear function.

Modelling is about better understanding of an object or phenomenon. Good models, including good cochlear models, are as simple as possible while still reproducing the behaviours of interest. Simple cochlear models can be used to gain useful information and insights to categorise our understanding of this complex and sophisticated sensory organ. These models can provide a simple framework to explain how the cochlear functions, and how the results of different experiments are linked to these functions (De Boer, 1996).

Ultimately, these insights will lead to better methods of diagnosis, and improved methods of treatment for hearing impairments. 


\subsection{Summary and Conclusions of Present Work}

Electrochemical activities inside the cochlea maintain resting potentials and the circulation of current inside the cochlea. External mechanical stimuli affect the circulation by changing the current that passes through mechanoelectrical transduction channels (Nin et al., 2012a). To investigate mechanoelectrical and electrochemical activities inside the cochlea, the changes in currents and potentials can be monitored by proper instruments. One of the electrical signals which is produced during this process is the cochlear microphonic. This signal has not received much attention since its discovery eighty years ago (Cheatham et al., 2011). The cochlear microphonic reflects mechanical activities and the excitation process of generating them. Probing the generation of the cochlear microphonic therefore can shed more light on the mechanisms involved in cochlear functions. The generation of the cochlear microphonic by endocochlear electromechanical activities and several clinical applications of the cochlear microphonic were discussed in Chapter 2.

This thesis presented an electromechanical model to link electrical and mechanical aspects of cochlear functions. The proposed integrated electromechanical model of the cochlea was constructed by embedding a nonlinear model of the outer hair cells in a electrical network of the organ of Corti and combining it with a onedimensional cochlear model. Different elements of the model and integrations were addressed in Chapter 3. Some of other relevant cochlear modelling approaches were also reviewed in Chapter 3.

A physiological model can only be used for interpretation and future predictions if the model is appropriately verified against experimental data. To this end, the electromechanical model developed in this thesis was tested and verified quantitatively and qualitatively in Chapter 4 using a variety of experimental data available on basilar membrane vibrations and on electrical activities inside the organ of Corti. The results of the model demonstrate that the amplification caused by electromotility of the outer hair cells, which is called the cochlear amplifier, makes the cochlea an excellent frequency analyser, and enhances the 
input stimulus. Consequently, even quiet sounds (very low amplitude stimuli) can be more effectively encoded by the inner hair cells, and more readily conceived by the brain. Without the active mechanism (i.e., cochlea amplifier) inside the cochlea, the dynamic range and frequency selectivity of hearing is reduced dramatically. In addition, we observed that the cochlea amplifier did not noticeably change the amplitude of the cochlear microphonic, which is consistent with experimental data provided by Liberman et al. (2002); Cheatham et al. (2011). The close match between mechanical and electrical outcomes of the model and experimental measurements validates the model, and suggests that the proposed model warrants further investigations.

The details of the matrices of the state-space representation of the model used in Chapter 4 are provided in Appendix A. The circuit analogy was used for analysis of the proposed model. The details of this approach are reported in Appendix B. The parameter values were carefully chosen by using available relevant data in Appendix C.

In mammals, the electrical potential recorded from the scala tympani are less sharply tuned than the basilar membrane tuning curves (Gelfand, 2010, Chapter 4).

It is commonly assumed that when an electrode is inserted at a particular point inside the scala media, the microphonic potentials of neighbouring hair cells may have different phases, and cancel each other, resulting in a relatively broad tuning curve (Patuzzi, 1987; Fridberger et al., 2004; He et al., 2012a).

To investigate the discrepancy between the tuning curves of basilar membrane and those of cochlear microphonic, and the effect of phase cancellation of adjacent hair cells on the broadness of the cochlear microphonic tuning curves, we devised an experiment reported in Chapter 5. We explored the potential effect of adjacent hair cells (i.e., longitudinal phase cancellation) on the broadness of the cochlear microphonic tuning curves in different locations. The results of the experiment indicated that active longitudinal coupling (i.e., coupling with active adjacent outer hair cells) slightly changed the broadness of the CM tuning curves.

The results also demonstrated that there is a $\pi$ phase difference between the 
potentials produced by the hair bundle $V_{\mathrm{HB}}$ and the soma $V_{\mathrm{OHC}}$ near the place associated with the characteristic frequency based on place-frequency maps (i.e, the best place).

Therefore, it seems that the transversal phase cancellation (caused by the phase difference between the hair bundle $V_{\mathrm{HB}}$ and the soma $V_{\mathrm{OHC}}$ ) plays a far more important role than longitudinal phase cancellation in the broadness of the cochlear microphonic tuning curves. Moreover, we noticed that by increasing the modelled longitudinal resistance the cochlear microphonic curves exhibit sharper tuning. The results of Chapter 5 suggest that the passive network of the organ of Corti determines the sharpness of the cochlear microphonic tuning curves.

There is evidence that in the mammalian cochleae, self-standing oscillation of the basilar membrane in the cochlea can cause vibration of the ear drum, and produce spontaneous narrow-band air pressure fluctuations in the ear canal. These spontaneous fluctuations are known as spontaneous otoacoustic emissions (Robles and Ruggero, 2001).

Small rapid perturbations in feedback gain have been proposed to be the generation source of self-standing oscillations of the basilar membrane (Nobili et al., 2003; Elliott et al., 2007; Rapson et al., 2012). Accordingly in Chapter 6, we hypothesised that the self-standing oscillation resulting from small rapid perturbations in feedback gain would produce spontaneous potentials in the cochlea. We demonstrated that according to the results of the model, a measurable spontaneous cochlear microphonic must exist in the human cochlea. The existence of this signal has not yet been reported. However, this spontaneous electrical signal could play an important role in auditory research. Successful or unsuccessful recording of this signal will indicate whether previous hypotheses about the generation source of spontaneous otoacoustic emissions are valid or should be amended. In addition according to the proposed model spontaneous cochlear microphonic is basically an electrical analogue of spontaneous otoacoustic emissions. In certain circumstances, spontaneous cochlear microphonic may be more easily detected near its generation site with proper electrical instrumentation than is spontaneous otoacoustic emissions. 
Considering necessary simplifications, any cochlear model including our model suffers from some limitations in mimicking cochlea functions. Nevertheless, we believe that the proposed model is particularly useful and powerful due to the following reasons:

- The pertinent electrical and mechanical components of the mammalian cochlea are modelled in very simple but precise way. The model is the most detailed one-dimensional cochlear model including electrical coupling of the cochlea developed to date.

- In spite of its simplicity, the model can reproduce the relevant mechanical and electrical observations of the cochlea, and consequently pave the way for conducting further intuitive and rigorous investigations and predictions similar to that carried out in Chapters 5 and 6.

\subsection{Limitations of the model}

The proposed model incorporates electrical longitudinal coupling. However for simplicity, we assume, as do most cochlea models, that adjacent regions of the $\mathrm{BM}$ are mechanically uncoupled except for the energy propagation through the fluid. Recent studies show that longitudinal coupling may stabilise the cochlea and increase the BM sensitivities to acoustic stimuli. Hence effects of structural longitudinal coupling that we have ignored may be significant (Naidu and Mountain, 2001; Meaud and Grosh, 2010; Saremi and Stenfelt, 2013). By considering longitudinal coupling, future versions of the model may produce sharper BM tuning curves than the results presented in Fig. 4.4. Incorporating longitudinal coupling may also make the conditions for stability of the model different from what was shown in Chapter 6.

Although some measurements from mammalian cochleae suggest that somatic motility is the basis of cochlear amplification (Lagarde et al., 2008), effects of the hair bundle motility on cochlear amplifier may also be significant (Nin et al., 
2012b). Hair bundle motility is simplified in our model. Considering the hair bundle motility in future versions of the model may also affect the mechanical and electrical responses of the model.

As mentioned in Section 3.2.1, the mechanical responses of a cochlear model are not significantly affected by coiling. However coiling may affect electrical responses of the model. In our straight model, we assume that there is no significant conductance between turns of the cochlea. We could not find any measurement showing that there is conductance between turns of the cochlea. Coupling between turns could be measured by appropriate instrumentation and if it exists, incorporated in future versions of the model.

\subsection{Future Work}

In the following some intended extensions to the current work are outlined. The cochlear microphonic is usually considered as a passive response (see Section 4.4), so it has not been used as an index to determine OHC integrity (Withnell, 2001; Cheatham et al., 2011). The usefulness of the cochlear microphonic can be investigated by removing OHCs and determining the detectibly in the amplitude or phase of the cochlear microphonic.

The cochlear microphonic recorded at the round window is believed to be generated at the cochlear base (He et al., 2012a). By using the proposed model, signals and experiments may be designed in future studies to elicit response from apical locations using tones in high pass noise similar to the approach advocated by Chertoff et al. (2012).

Due to the anatomical arrangement of the OHCs and IHCs, they exhibit different vulnerabilities to mechanical stress such as exposure to a high level of impulse noise or prolonged exposure to loud noise (Borg et al., 1995; Hu, 2012). As previously mentioned in Section 2.5.2, the cochlear microphonic is mainly generated by the OHCs. The IHCs also contribute to this extracellular potential 
(Dallos, 1983; Withnell, 2001). The current research can be extended by including the IHCs in the electrical model, and checking their effects on the cochlear microphonic. The results may enable audiologists to better distinguish OHC hearing loss (sensitivity loss) from IHC hearing loss (clarity loss) (Killion and Niquette, 2000) for prescribing suitable hearing aids or treatments.

According to the prediction of the model in Chapter 6, the spontaneous cochlear microphonic seems to exist. This study therefore motivates experiments to determine the existence or non-existence of spontaneous cochlear microphonic in humans or experimental animals.

In our simulations, we noticed that even though imposed abrupt spatial irregularities cause self-sustained oscillations of the basilar membrane associated with the locations of irregularities, two separate but close spatial irregularities do not necessarily produce two separate oscillations along the basilar membrane. This observation may bear on the minimum frequency spacing expected from global standing wave theory reported by Shera (2003). This line of research is not directly related to the presented work but it seems fruitful. This may help in answering the open question of the origin of spontaneous otoacoustic emissions.

The proposed lumped model successfully reproduces, explains and predicts the certain features of the cochlea studied in this thesis. The presented ideas however can be examined and extended in future work using a three-dimensional finite element model ${ }^{1}$ of the cochlea including individual hair cells. A detailed threedimensional finite element model of the cochlea may provide more insights into electrical potentials and functions of individual hair cells.

\footnotetext{
${ }^{1}$ Finite element method is a flexible numerical method by which a geometrically complicated physical structure can be systematically divided into a number of discrete elements, and the entire behaviour of this structure can be approximated by a combination and interaction of these elements (Reddy, 2006, Chapter 1). This technique can be used to analyse a continuum such as a fluid, or a biological organ consisting of separate, discrete components (Kolston, 2000).
} 
CHAPTER 7. SUMMARY, CONCLUSIONS AND SUGGESTIONS FOR FURTHER WORK 


\section{Appendix A}

\section{State space formulation}

\section{A.1 State Space Matrices}

As mentioned in Chapter 4, the following variables were chosen as state variables: diaphragm variables $\left\{x_{\mathrm{d}}, v_{\mathrm{d}}\right\}$, middle-ear variables $\left\{x_{\mathrm{m}}, v_{\mathrm{m}}, x_{\mathrm{s}}, v_{\mathrm{s}}\right\}$, and the $6 \mathrm{~N}$ 
cochlear variables $\left\{\xi_{\mathrm{r}}(n), \dot{\xi}_{\mathrm{r}}(n), \xi_{\mathrm{o}}(n), \dot{\xi}_{\mathrm{o}}(n), V_{\mathrm{OHC}}(n), V_{\mathrm{HB}}(n)\right\}$.

$$
\left[\begin{array}{c}
v_{\mathrm{d}} \\
x_{\mathrm{d}} \\
v_{\mathrm{m}} \\
x_{\mathrm{m}} \\
v_{\mathrm{s}} \\
x_{\mathrm{s}} \\
\xi_{\mathrm{r}}(0) \\
\dot{\xi}_{\mathrm{r}}(0) \\
\xi_{\mathrm{o}}(0) \\
\dot{\xi}_{\mathrm{o}}(0) \\
V_{\mathrm{OHC}}(0) \\
V_{\mathrm{HB}}(0) \\
\vdots \\
\xi_{\mathrm{r}}(N) \\
\dot{\xi}_{\mathrm{r}}(n)(N) \\
\xi_{\mathrm{o}}(N) \\
\dot{\xi}_{\mathrm{o}}(N) \\
V_{\mathrm{OHC}}(N) \\
V_{\mathrm{HB}}(N)
\end{array}\right] \quad \dot{\mathbf{x}}=\left[\begin{array}{c}
\dot{v}_{\mathrm{d}} \\
\dot{x}_{\mathrm{d}} \\
\dot{v}_{\mathrm{m}} \\
\dot{x}_{\mathrm{m}} \\
\dot{v}_{\mathrm{s}} \\
\dot{x}_{\mathrm{s}} \\
\dot{\xi}_{\mathrm{r}}(0) \\
\ddot{\xi}_{\mathrm{r}}(0) \\
\dot{\xi}_{\mathrm{o}}(0) \\
\ddot{\xi}_{\mathrm{o}}(0) \\
\dot{V}_{\mathrm{OHC}}(0) \\
\dot{V}_{\mathrm{HB}}(0) \\
\vdots \\
\dot{\xi}_{\mathrm{r}}(N) \\
\ddot{\xi}_{\mathrm{r}}(N) \\
\dot{\xi}_{\mathrm{o}}(N) \\
\ddot{\xi}_{\mathrm{o}}(N) \\
\dot{V}_{\mathrm{OHC}}(N) \\
\dot{V}_{\mathrm{HB}}(N)
\end{array}\right]
$$

In the following, the details of the matrices previously introduced in Chapter 4 are provided.

\section{Earphone Diaphragm, Eardrum and Middle ear $\left(\mathrm{A}_{\mathrm{ME}}\right)$}

The matrix $\mathbf{A}_{\mathrm{ME}}$ is a square matrix of order $6 N+6$ which represents equilibrium equations for the earphone diaphragm, eardrum and middle ear ( Fig. 3.6 (A) and (B) (Equations (3.4) to (3.6c) ) in the first six rows and columns and all other elements of this matrix are zeros. 


$$
\begin{aligned}
& \mathbf{A}_{\mathrm{ME}}= \\
& {\left[\begin{array}{cccccccc}
-\frac{R_{\mathrm{d}}}{M_{\mathrm{d}}} & -\frac{K_{\mathrm{d}}+K_{\mathrm{c}} A_{\mathrm{d}}^{2}}{M_{\mathrm{d}}} & 0 & \frac{K_{\mathrm{c}} A_{\mathrm{d}} A_{\mathrm{e}}}{M_{\mathrm{d}}} & 0 & 0 & 0 & \ldots \\
1 & 0 & 0 & 0 & 0 & 0 & 0 & \ldots \\
0 & \frac{K_{\mathrm{c}} A_{\mathrm{d}} A_{\mathrm{e}}}{M_{\mathrm{m}}} & \frac{-R_{\mathrm{m}}+g^{2} R_{\mathrm{i}}}{M_{\mathrm{m}}} & \frac{-K_{\mathrm{m}}-g^{2} K_{\mathrm{i}}-K_{\mathrm{c}} A_{\mathrm{e}}^{2}}{M_{\mathrm{m}}} & \frac{g R_{\mathrm{i}}}{M_{\mathrm{m}}} & \frac{g K_{\mathrm{i}}}{M_{\mathrm{m}}} & 0 & \ldots \\
0 & 0 & 1 & 0 & 0 & 0 & \ldots & \\
0 & 0 & \frac{g R_{\mathrm{i}}}{M_{\mathrm{s}}+M_{\mathrm{r}}} & \frac{g K_{\mathrm{i}}}{M_{\mathrm{s}}+M_{\mathrm{r}}} & \frac{R_{\mathrm{s}}+R_{\mathrm{r}}-R_{\mathrm{i}}}{M_{\mathrm{s}}+M_{\mathrm{r}}} & \frac{K_{\mathrm{s}}+K_{\mathrm{r}}-K_{\mathrm{i}}}{M_{\mathrm{s}}+M_{\mathrm{r}}} & 0 & \ldots \\
0 & 0 & 0 & 0 & 0 & 1 & 0 & \ldots \\
0 & 0 & 0 & 0 & 0 & 0 & 0 & \ldots \\
\vdots & \vdots & \vdots & \vdots & \vdots & \vdots & \ldots &
\end{array}\right]}
\end{aligned}
$$

\section{Micromechanics $\left(\mathbf{A}_{\mathbf{C}}\right)$}

The matrix $\mathbf{A}_{\mathrm{C}}$ is a square matrix of order $6 N+6$ which presents the micromechanical part of the PCM ( Fig. 3.6 (D), equations (4.4a) to (4.4d)).

$\mathbf{A}_{\mathrm{C}}(n)=$

$$
\left[\begin{array}{cccccc}
-\frac{k(n)}{m(n)} & -\frac{r(n)}{m(n)} & \frac{K(n)}{M(n)}+\frac{1}{C_{g}(n) T(n)^{2} M}-\frac{k(n)}{m(n)} & \frac{R(n)}{M(n)}-\frac{r(n)}{m(n)} & -\frac{1}{T(n) M(n)} & 0 \\
0 & 0 & 1 & 0 & 0 & 0 \\
0 & 0 & -\frac{K(n)}{M(n)}-\frac{1}{C_{g} T(n)^{2} M(n)} & -\frac{R(n)}{M(n)} & \frac{1}{T(n) M(n)} & 0 \\
0 & 0 & 0 & 0 & 0 & 0 \\
0 & 0 & 0 & 0 & 0 & 0 \\
0 & 0 & 0 & 0 & 0 & 0
\end{array}\right]
$$

and

$$
\mathbf{A}_{\mathrm{C}}=\left[\begin{array}{cccccc}
0_{6 \times 6} & \cdots & & & \cdots \\
\vdots & \mathbf{A}_{\mathrm{C}}(0) & 0_{6 \times 6} & & \cdots \\
\vdots & 0_{6 \times 6} & \mathbf{A}_{\mathrm{C}}(1) & 0 & 0 & 0 \\
0_{6 \times 6} & 0_{6 \times 6} & 0_{6 \times 6} \cdot & \cdots & \\
\vdots & \vdots & \vdots & & \mathbf{A}_{\mathrm{C}}(N)
\end{array}\right]
$$




\section{Fluid pressures $\left(\mathrm{A}_{\mathbf{P}}\right)$}

The square matrix $\mathbf{A}_{\mathrm{P}}$ of order $6 N+6$ is constructed as follows: 


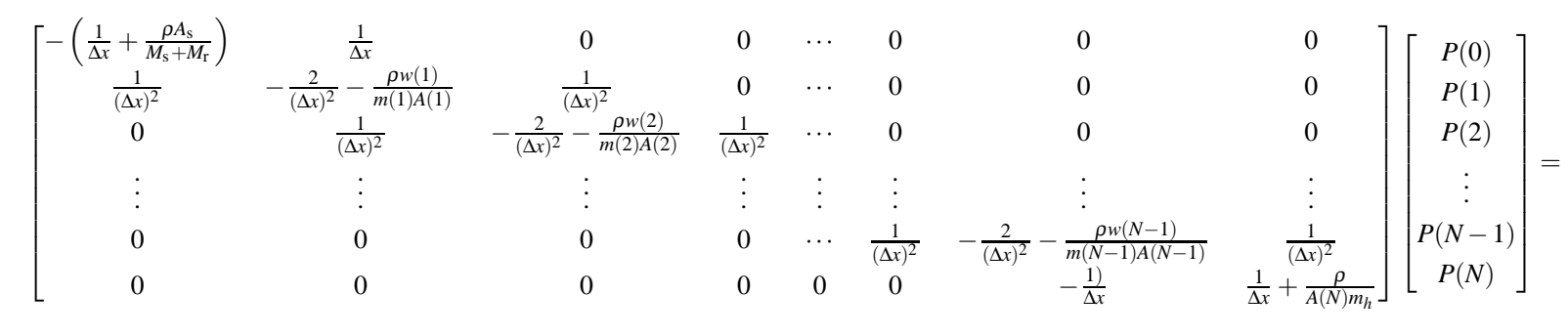

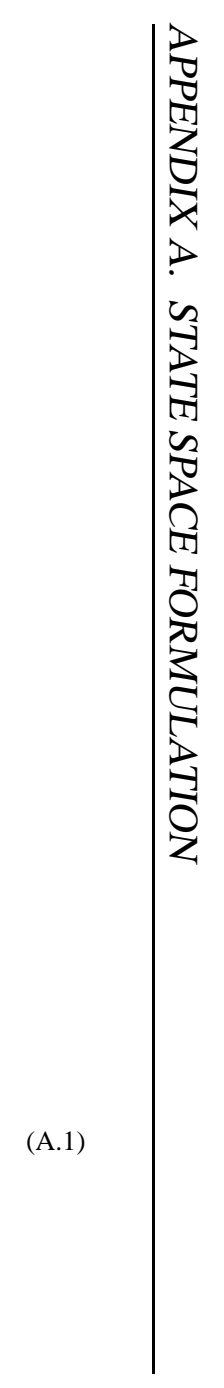


$\mathbf{P}_{\dot{\xi}_{\mathrm{r}}}$ is a $(6 N+6) \times(N)$ matrix which can be constructed to rearrange the pressure values to a format suitable for combination with equation (4.4b).

$$
\mathbf{A}_{\mathrm{P}}=\mathbf{P}_{\dot{\xi}_{\mathrm{r}}}\left(\mathbf{D}_{2}-\hat{\mathbf{D}}_{2}\right)^{-1} \mathbf{L}
$$

where $\hat{\mathbf{D}}=\operatorname{Diagonal}\left(\frac{\rho}{A} w\right)$

\section{Electrical Coupling $\left(\mathbf{A}_{\mathbf{G}}\right)$}

By considering vector $V$ as follows:

$$
\mathbf{V}=\left[\begin{array}{c}
V_{\mathrm{sv}}(0) \\
V_{\mathrm{sm}}(0) \\
V_{\mathrm{st}}(0) \\
\dot{V}_{\mathrm{HB}}(0) \\
\dot{V}_{\mathrm{OHC}}(0) \\
\vdots \\
V_{\mathrm{sv}}(N) \\
V_{\mathrm{sm}}(N) \\
V_{\mathrm{st}}(N) \\
\dot{V}_{\mathrm{hb}}(N) \\
\dot{V}_{\mathrm{OHC}}(N)
\end{array}\right] \quad \mathbf{V}(i)=\left[\begin{array}{c}
V_{\mathrm{sv}}(i) \\
V_{\mathrm{sm}}(i) \\
V_{\mathrm{st}}(i) \\
\dot{V}_{\mathrm{HB}}(i) \\
\dot{V}_{\mathrm{OHC}}(i)
\end{array}\right]
$$

equations (4.13) to (4.17) can be written in matrix form as follows: 
for simplicity $G=\frac{1}{R}$ has been used in the matrix.

$G_{1}(i)=\left[\begin{array}{ccccc}V_{\mathrm{sv}}(i-1) & V_{\mathrm{sm}}(i-1) & V_{\mathrm{st}}(i-1) & \dot{V}_{\mathrm{HB}}(i-1) & \dot{V}_{\mathrm{OHC}}(i-1) \\ \hline-G_{8}(i) & 0 & 0 & 0 & 0 \\ 0 & -G_{9}(i) & 0 & 0 & 0 \\ 0 & 0 & -G_{11}(i) & 0 & 0 \\ 0 & 0 & 0 & 0 & 0 \\ 0 & 0 & 0 & 0 & 0\end{array}\right]$

$\Xi$

$G_{2}(i)=\left[\begin{array}{ccccc}V_{\mathrm{sv}}(i) & V_{\mathrm{sm}}(i) & V_{\mathrm{st}}(i) & \dot{V}_{\mathrm{HB}}(i) & \dot{V}_{\mathrm{OHC}}(i) \\ \hline G_{8}(i+1)+G_{8}(i)+G_{1}(i)+G_{2}(i) & -G_{2}(i) & 0 & 0 & 0 \\ -G_{2}(i) & G_{9}(i+1)+G_{9}(i)+G_{2}(i)+G_{6}(i) & 0 & C_{3}(i) & 0 \\ 0 & 0 & G_{11}(i)+G_{11}(i+1)+G_{5}(i) & 0 & -C_{4}(i) \\ 0 & 0 & 0 & C_{3}(i) & -C_{4}(i) \\ 0 & 1 & -1 & 0 & 0\end{array}\right]$

$G_{3}(i)=\left[\begin{array}{ccccc}V_{\mathrm{sv}}(i+1) & V_{\mathrm{sm}}(i+1) & V_{\mathrm{st}}(i+1) & \dot{V}_{\mathrm{HB}}(i+1) & \dot{V}_{\mathrm{OHC}}(i+1) \\ \hline-G_{8}(i+1) & 0 & 0 & 0 & 0 \\ 0 & -G_{9}(i+1) & 0 & 0 & 0 \\ 0 & 0 & -G_{11}(i+1) & 0 & 0 \\ 0 & 0 & 0 & 0 & 0 \\ 0 & 0 & 0 & 0 & 0\end{array}\right]$

$$
C_{\mathrm{E}}(i)=\left[\begin{array}{c}
0 \\
G_{6}(i) V_{2}(i)-i_{\mathrm{r}}(i)-G_{3} V_{\mathrm{HB}}(i) \\
i_{\mathrm{Q}}(i)+G_{4}(i) V_{\mathrm{OHC}}(i) \\
-i_{\mathrm{r}}(i)+i_{\mathrm{Q}}(i)+G_{4} V_{\mathrm{OHC}}(i)-G_{3} V_{\mathrm{HB}}(i) \\
V_{\mathrm{HB}}(i)+V_{\mathrm{OHC}}(i)-V_{1}(i)
\end{array}\right]
$$




$$
\begin{aligned}
& {\left[\begin{array}{lll}
G_{1}(i) & G_{2}(i) & G_{3}(i)
\end{array}\right]\left[\begin{array}{c}
\mathbf{V}(i-1) \\
\mathbf{V}(i) \\
\mathbf{V}(i+1)
\end{array}\right]=C_{\mathrm{E}}(i)} \\
& \mathbf{G}=\left[\begin{array}{ccccccc}
G_{2}(0) & G_{3}(0) & 0 & \ldots & 0 & 0 & 0 \\
G_{1}(1) & G_{2}(1) & G_{3}(1) & \cdots & 0 & 0 & 0 \\
0 & \ddots & \ddots & \cdots & 0 & 0 & 0 \\
0 & 0 & 0 & \cdots & G_{1}(N-1) & G_{2}(N-1) & G_{3}(N-1) \\
0 & 0 & 0 & \cdots & 0 & G_{1}(N) & G_{2}(N)
\end{array}\right] \\
& \mathbf{G V}=\left[\begin{array}{c}
C_{\mathrm{E}}(0) \\
\vdots \\
C_{\mathrm{E}}(N)
\end{array}\right]
\end{aligned}
$$

In these matrices, the resistances were replaced by the conductances $(G=1 / R)$ : By using linearized form of $i_{\mathrm{r}}$, Equation (A.3) can be written as:

$$
\mathbf{G V}=\mathbf{E x}
$$

where $\mathbf{E}$ constructs the combination of the state variables of the right side of (A.3). Matrix $\mathbf{K}$ can be made to extract the $2 \times N$ state variables $\dot{V}_{\mathrm{OHC}}$ and $\dot{V}_{\mathrm{HB}}$. Therefore:

$$
\mathbf{A}_{\mathrm{G}}=\mathbf{K G}^{-1} \mathbf{E}
$$

and finally A can be calculated using (4.20). 


\section{Appendix B}

\section{Using Circuit Analogies for Analysis of Cochlear Models}

As shown in chapter 3, interactions between different parts of the cochlear model can be represented by differential equations. spatial discretisation can be used to simplify the particular differential equations to ordinary differential equations that can be solved by conventional numerical methods. However solving these equations demands high computational costs, especially as the time scales of different components of the model can be different by several order of magnitudes, i.e., the system can be described as stiff (Miranker and Miranker, 2002, Chapter 1). Various technique such as the use of a hybrid direct-iterative solver, and using noninverted form of matrices have been suggested for a numerical solution of cochlear models (Bertaccini and Sisto, 2011; Teal et al., 2011; Rapson et al., 2012).

Another efficient alternative method for facilitating computation is to use system analogies. In this method, analogies between different phenomena are utilised to make the whole system more understandable and analysable. Using a circuit analogy is often popular among scientists and engineers (Senturia, 2001).

Using electrical circuits for modelling the cochlea (Wegel and Lane, 1924) dates back several decades, before the development of digital computers and before active behaviour was observed in the cochlea (Rhode, 1971). Even though many 
researchers use a circuit analogy to describe their cochlear models (Wegel and Lane, 1924; Neely, 1993), circuit simulator software has rarely been used to analyse a cochlear model.

In addition to performing the frequency domain analysis, which has been employed for analysing cochlear models (Lu et al., 2006; Kim et al., 2011), circuit simulators are capable of performing time domain analysis of a circuit. This ability of circuit simulators has not been reported for analysing nonlinear cochlear models.

In the following section the equivalent electric circuit of each part of the proposed model will be developed and analysed with SPICE (Simulation Program with Integrated Circuit Emphasis). The results of this method have been demonstrated in chapters 4, 5 and 6.

\section{B.1 Materials and Methods}

The analogy between simple mechanical and electrical systems is straightforward Senturia (2001). Consider the mechanical system in Fig. B.1. Newton's second law mandates the following equation:

$$
f=K x+M \frac{d^{2} x}{d t^{2}}+B \frac{d x}{d t}
$$

By using Kirchhoff's current and voltage laws for the electrical circuit in Fig. B.1, the following equation can be written:

$$
v=\frac{1}{C} \int_{0}^{t} i d t+L \frac{d i}{d t}+R i
$$

If we assume that the velocity $\dot{x}$ is equivalent to the electrical current $i$ (the velocity and current go through a series of elements and are called through variables), the force $f$ is equivalent to the voltage $v$ (the force and voltage are called across variables) (Senturia, 2001). We can represent these equivalents as $\dot{x} \Leftrightarrow i$ and $f \Leftrightarrow v$. Using this notation, $B \Leftrightarrow R, M \Leftrightarrow L$ and $K \Leftrightarrow 1 / C$. The differential equations (B.1) and (B.2) are equivalent and the mechanical system and electrical circuit in 


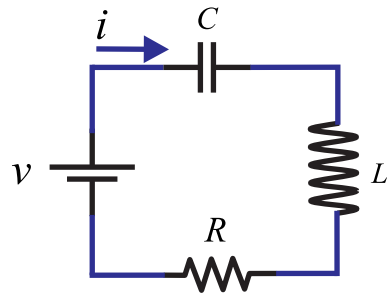

Electrical Circuit

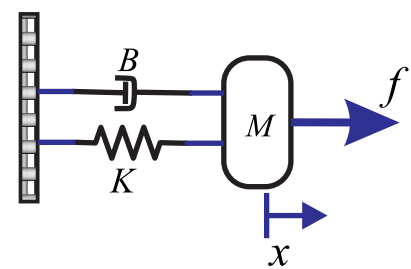

Mechanical System

Figure B.1: (a) A mechanical system with mass $M$, spring constant $K$ and damper of damping coefficient $B$. (b) An equivalent electrical circuit of (a) with the resistance $R$, the inductance $L$ and the capacitance $C$. $i$ represents the electrical current in the electrical system and $x$ represents the displacement.

Fig. B. 1 become equivalent. This analogy can be extended to acoustic and fluid systems.

\section{B.1.1 Equivalent Circuit of the Sound Source and Middle Ear Models}

The models of the sound source and middle ear are composed of simple acoustical and mechanical components which can be converted to equivalent circuits by exploiting the approach of the previous section. Those models also include lever gains and the pressures on changing cross sectional areas, both of which can be represented by ideal transformers. Fig. B.2 demonstrates the equivalent circuit for this part of the model. The stimulus force $f(t)$ is substituted by an equivalent voltage source. All components of the middle ear are replaced by their equivalent electrical elements. Therefore, equations (3.4) to (3.6c) are valid for this circuit.

\section{B.1.2 Equivalent Circuit of Cochlear Macromechanics}

By writing the finite difference approximation for (3.8) and (3.9), assuming that $P(n)=P(n \Delta x, t), U(n)=U(n \Delta x, t)$ and $\xi_{\mathrm{r}}(n)=\xi_{\mathrm{r}}(n \Delta x, t)$, the following 


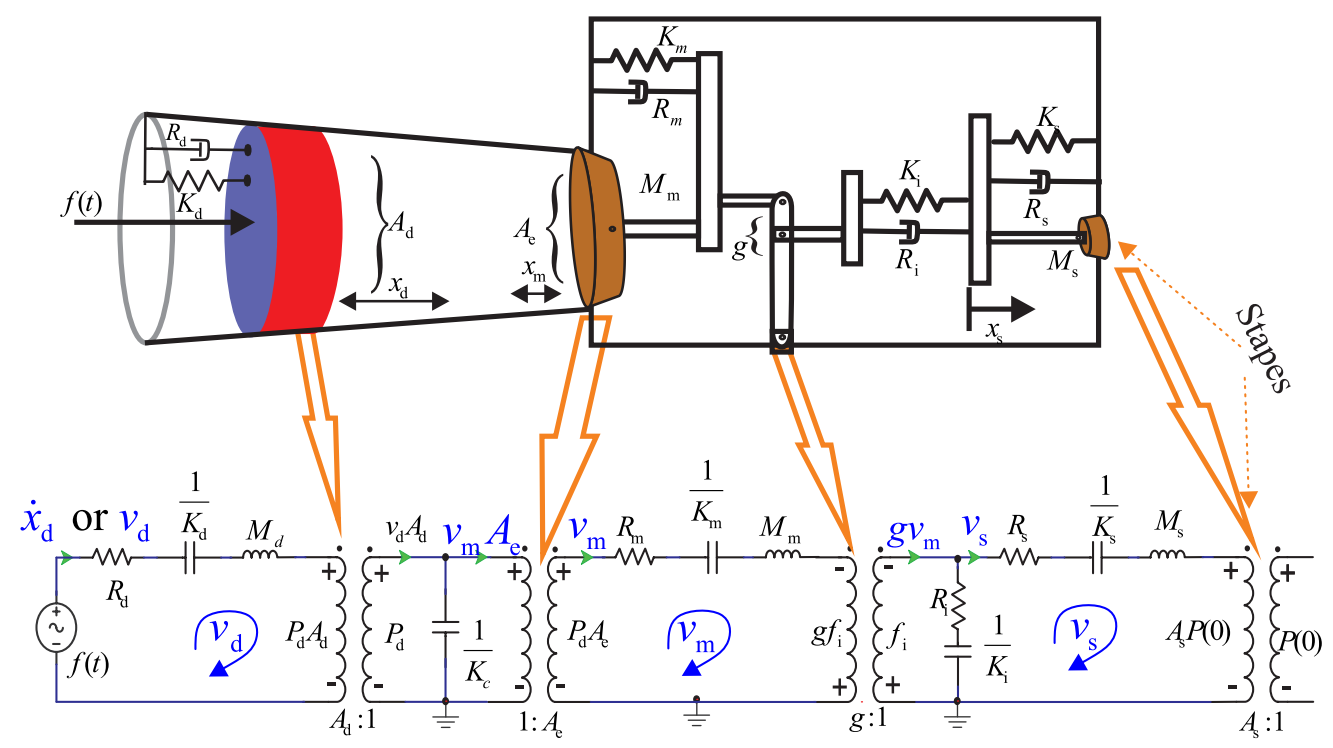

Figure B.2: Equivalent circuit of the sound source and middle ear models

equations are derived:

$$
\begin{aligned}
& \frac{P(n)-P(n-1)}{\Delta x}=-\frac{\rho}{A(n)} \dot{U} \\
& \frac{U(n)-U(n-1)}{\Delta x}=w \dot{\xi}_{\mathrm{r}}(n)
\end{aligned}
$$

The fluid volume velocity is assumed to be equivalent to the current and the fluid pressure is assumed to be equivalent to the voltage, so (B.3) represents an inductor in the circuit equivalent, with the inductance $L(n)=\frac{\rho}{A(n)} \Delta x$. By using same approach, (3.11) represents an inductor with the inductance $L=\frac{\rho}{A_{\mathrm{s}}} \Delta x$ and (4.10) represents two resistors at the helicotrema with resistances $R_{1}=\frac{\rho}{A(N-1) m_{\mathrm{h}}}$ and $R_{2}=\frac{1}{\Delta x}$.

\section{B.1.3 Equivalent Circuit of Cochlear Micromechanics}

Equations (3.13) and (3.14) represent the cochlear micromechanical part of the proposed model. Both equations can be easily represented by an electrical circuit. 


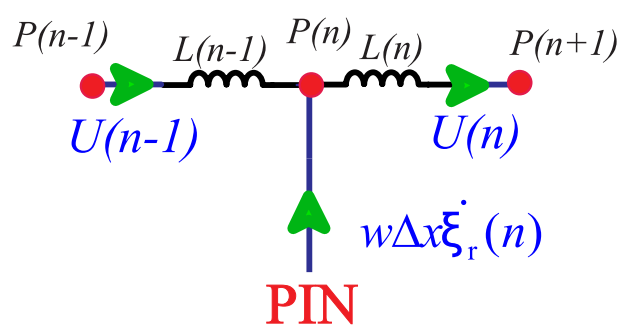

Figure B.3: The equivalent circuit for equations (B.3) and (B.4). Equation (B.3) represents an inductor, with the inductance $L(n)=\frac{\rho}{A(n)} \Delta x$. Equation (B.4) dictates that the current entering the node $P(n)$ should be $w \Delta x \dot{\xi}_{\mathrm{r}}(n)$. This circuit was connected to the micromechanical circuit of the model via a node which is labelled "PIN" as shown in this circuit.

It should be also taken into account that the current entering to the node $P(n)$ is $w \Delta x \dot{\xi}_{\mathrm{r}}$. Therefore, the electrical component values should be adjusted in order to satisfy (B.4). The resulting circuit is shown in Fig. B.4.

By considering equation (3.17), the dependent voltage source $f_{\mathrm{OHC}}{ }^{1}$ relies on $\tilde{V}$ and $V_{\text {OHC }}$. $\tilde{V}$ (refer to 3.4.1, $\tilde{V}=\left(T C_{\mathrm{g}}\right)^{-1} \xi_{\mathrm{o}}$ ) can be obtained by taking the integral of $\dot{\xi}_{\mathrm{o}}$. This procedure can be accomplished by using a dependent current source and a capacitor in the circuit equivalent (see Fig. B.4). $V_{\mathrm{OHC}}(n)$ is the electrical potential of $R_{4}(n)$ in the potential coupling (see Fig. 3.7). $i_{\mathrm{r}}(n)$ depends on the displacement and velocity of the RL. $\dot{\xi}_{\text {r }}$ can be obtained by using the current representation the velocity of the $\mathrm{BM}$ and $\mathrm{OHC}$ branches. $\xi_{\mathrm{r}}$ can be calculated by a dependent current source and a capacitor in the circuit equivalent (see Fig. B.4). Therefore, $\eta=\alpha_{\mathrm{v}} \dot{\xi}_{\mathrm{r}}(n)+V(\mathrm{P} 6,0)$.

\section{B.2 SPICE implementation}

For implementing the circuit equivalent of the model, LTspice ${ }^{2}$ and its netlist syntax have been used. Matlab ${ }^{\circledR}$ has been used to create the net-list files.

\footnotetext{
${ }^{1}$ Equivalent to the forces which are generated by the OHCs against $\mathrm{OHC}$ load impedances

${ }^{2}$ LTspice $^{\circledR}$ is a free SPICE software from Linear Technology Corporation (http://www.linear.com/designtools/software/).
} 


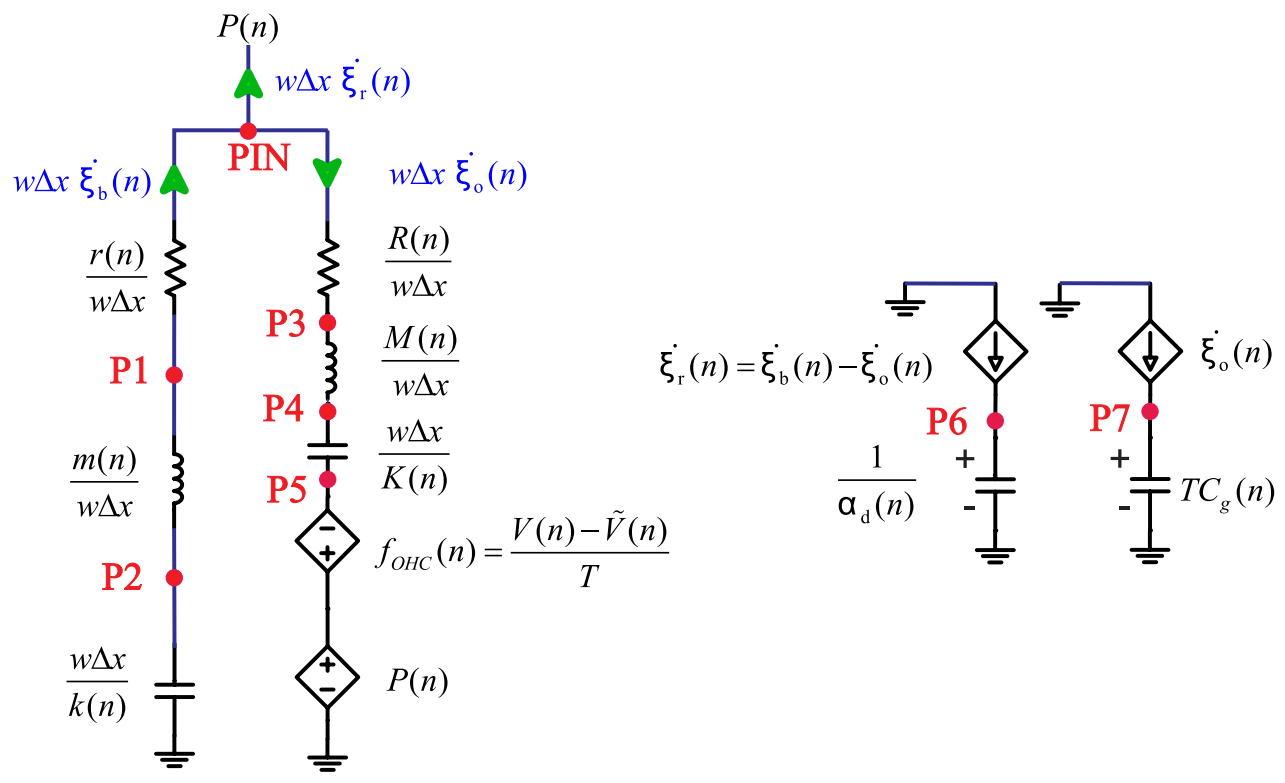

Figure B.4: This circuit represents equations (3.13), (3.14) and (3.17). For satisfying equation (B.4), the electrical component values are adjusted (see text). $V_{i}=\alpha_{\mathrm{d}} \xi_{\mathrm{r}}(i)$ and $\tilde{V}(x)=$ $\left(C_{g} T\right)^{-1} \xi_{o}(x)$. The nodes "PIN" and "P1" to "P7" are used in the SPICE implementation of the model.

The circuit equivalent of cochlear micromechanics and electrical coupling consist of repetitive circuitry which is easier to implement if enclosed in a sub-circuit. The net-list of this sub-circuit is listed as follows:

.SUBCKT CROSS_SECTION PIN VB VO VHB VOHC SV SM ST COMON

*The sub-circuit, CROSS_SECTION encases a lumped model

*of a cross section of the organ of Corti.

$*$ PIN is the connection node to the inductors

*representing the fluid pressure.

$* \mathrm{VB}$ and $\mathrm{VO}$ is the output current nodes that calculates

*the $\mathrm{BM}$ and the $\mathrm{OHC}$ velocities, respectively.

$* \mathrm{VHB}, \mathrm{VOHC}, \mathrm{SV}, \mathrm{SM}$ and ST are the potential of the hair 
*bundle,OHC soma, scala vestibuli, scala media and *scala tympani, respectively.

$\begin{array}{llll}*--- & \text { Mechanical } & \text { part---- } \\ \text { CK_B } & \text { COMON } & \text { P2 } & \{\mathrm{w} * \mathrm{dx} / \mathrm{KB}\} \\ \text { LM_B } & \text { P2 } & \text { P1 } & \{\mathrm{MB} /(\mathrm{w} * \mathrm{dx})\} \\ \text { RR_B } & \text { P1 } & \text { PIN } & \{\mathrm{RB} /(\mathrm{w} * \mathrm{dx})\}\end{array}$

*CK_B, LM_B and RR_B are adjusted values for *stiffness, mass and resistance of the BM *per unit area, respectively.

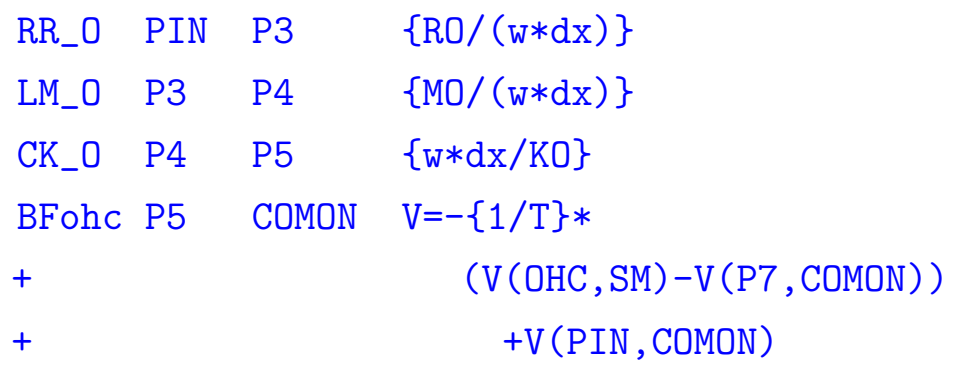

$* \mathrm{CK}_{-} \mathrm{O}$, LM_O and RR_O are adjusted values

*for stiffness, mass and resistance of

*the OHC load, respectively.

$* \mathrm{~V}(\mathrm{OHC}, \mathrm{SM})$ is the potential of the soma (V_OHC).

$* \mathrm{~V}(\mathrm{P} 7, \mathrm{COMON})$ is $\mathrm{V} \_$tilda.

B4 COMON P6 $I=1 /(\mathrm{w} * \mathrm{dx}) *\left(\mathrm{I}\left(\mathrm{RR} \_\mathrm{B}\right)-\mathrm{I}\left(\mathrm{RR} \_0\right)\right)$

CCALPHAD P6 COMON $\{1 /$ alpha_d $\}$ 
BFF2 COMON P7 $\mathrm{I}=1 /(\mathrm{w} * \mathrm{dx}) * \mathrm{I}\left(\mathrm{RR} \_0\right)$

CCGT P7 COMON $\{\mathrm{T} * \mathrm{Cg}\}$

$* \mathrm{Cg}$ is the gaining capacitor.

$* \mathrm{~V}(\mathrm{P} 7, \mathrm{COMON})$ is the V_tilda;

*----Electrical Part---------------------

RR_1 COMON SV $\{R 1\}$

RR_2 SV SM $\{R 2\}$

RR_6 SM COMON $\{R 6\}$

*----- Hair Bundle-------

* Ir is the MET Channel current.

** linear for .ac .tran mode

BIr SM OHC I=(1/(w*dx)*alpha_V*(I (RR_B)

$\left.\left.+\quad-\mathrm{I}\left(\mathrm{RR}_{-} \mathrm{O}\right)\right)+\mathrm{V}(\mathrm{P} 6, \mathrm{COMON})\right)$

** nonlinear only for transient mode

$* \mathrm{BIr} \mathrm{SM} \mathrm{OHC} *+\mathrm{I}=\mathrm{IMAX} / 2 * \tanh (2 *(1 /(\{\mathrm{W}\} *\{\mathrm{DX}\}) * a l$ pha_V$*$

$\left.\left.* \quad+\left(\mathrm{I}\left(\mathrm{RR} \_\mathrm{B}\right)-\mathrm{I}\left(\mathrm{RR} \_\mathrm{O}\right)\right)+\mathrm{V}(\mathrm{P} 6, \mathrm{COMON})\right) /\{\mathrm{IMAX}\}\right)$

RR_3 SM OHC $\{\mathrm{R} 3\}$

CC_3 SM OHC $\{\mathrm{C} 3\}$

*----- OHC SOMA------

BF4 OHC ST I $=\mathrm{I}\left(\mathrm{RR}_{-} \mathrm{O}\right) *\{1 / \mathrm{T} * 1 /(\mathrm{W} * \mathrm{DX})\}$

CC_4 OHC ST $\{\mathrm{C} 4\}$

RR_4 OHC ST $\{R 4\}$

RR_5 ST COMON $\{R 5\}$ 
*CURRENT SOURCES FOR ACCESSING VEL_O AND VEL_B

BFVELO COMON VO $\mathrm{I}=1 /(\mathrm{w} * \mathrm{dx}) * \mathrm{I}\left(\mathrm{RR} \_0\right)$

BFVELB COMON VB $\mathrm{I}=1 /(\mathrm{w} * \mathrm{dx}) * \mathrm{I}\left(\mathrm{RR} \_\mathrm{B}\right)$

*CURRENT SOURCES FOR ACCESSING V_ohc AND V_hb

BVOL_HB COMON VHB $\mathrm{I}=\{\mathrm{R} 3\} * \mathrm{I}\left(\mathrm{RR} \_3\right)$

BVOL_OHC COMON VOHC I $=\{\mathrm{R} 4\} * \mathrm{I}\left(\mathrm{RR} \_4\right)$

In a SPICE netlist, ".SUBCKT" defines a sub-circuit and comment lines start with an asterisk (*). The ' + ' (plus) sign at the beginning of a line means that the second line is a continuation of the previous line (Quarles et al., 1993).

The suggested procedure for converting the proposed model to an electrical circuit can be used as a framework to represent any one-dimensional (1D) model of the cochlea, including nonlinearity by electrical circuits. These circuits then can be readily analysed using SPICE.

\section{B.3 Discussion and Summary}

In general, a cochlear model is a large system of ordinary differential equations. In a realistic model of the cochlea, the nonlinearity should also be considered in these equations. Accordingly, solving these differential equations becomes cumbersome. An attractive alternative approach for solving these equations is to use a circuit analogy and using a powerful circuit simulator such as SPICE to solve them. SPICE can also report implementation bugs in a circuit.

Although there is in principle no different between using circuit analogy implementation of a cochlear model or implementing it using other methods, in practice the use of circuit analogies is likely to produce more reliable results, as there are 
less likely to be errors resulting from implementation.

In this appendix, equivalent circuits have been used to derive the differential equations of the proposed model. These resulting circuits can be readily implemented in circuit analysis software such as SPICE. The approach which has been discussed here can be applied to any one-dimension cochlear model to allow the use of circuit analysis software to analyse them.

Circuit analysis software has been developed to analyse large, complex and nonlinear electrical circuits thoroughly, quickly and precisely (Vlasak, 2012). These capabilities have been used in this project to analyse circuit representation of the model. Both time domain and frequency domain simulations can be easily performed on circuit equivalents of cochlear models.

Many researchers have investigated building a silicon cochlea (Watts, 1993; Wen, 2006; Hamilton et al., 2008) which can be considered a starting point of building a very robust and efficient speech recognition system similar to the human cochlea. The proposed frame work facilitates further developing of silicon cochlea research. 


\section{Appendix C}

\section{Parameter Selection and Calibration}

The mammalian cochlea has very intricate structure and outstanding performance. These features make it very challenging to model, and consequently a wide variety of configurations has been being proposed to model various specific functions of the cochlea (see Section 3.2.3). In addition to variation in configurations, the selection of parameter values for each configuration is very challenging.

Modelling work greatly depends on available data from experimental measurements. However a complete set of measurements for modelling the cochlea of one mammalian species is not available and most the time is only restricted to a few places along the cochlear partition. Consequently for a detailed model of the cochlea of one species, many assumptions inevitably must be included (Maoiléidigh and Hudspeth, 2013). Current technology also precludes the measurement of all parameter values for different elements of a cochlear model, and a common approach is to determine the parameter values of elements of a model by using available measurements and so reduce the number of free (immeasurable) parameters to as few as possible (Duifhuis, 2012, Chapter 3 ).

Some parameter values of a cochlear model vary with longitudinal position. Greenwood's frequency-place map (see page 58) constrains many of these to be exponentially dependent on the distance from the base (Liberman, 1982; 
Greenwood, 1990b).

\section{C.1 Mechanical Parameter Values}

In the current work, the mechanical parameter values have been taken from Liu and Neely $(2010,2009)$ and listed in Table. C.1 and Table. C.2.

Table C.1: Parameter values for the earphone diaphragm and the middle-ear mechanics.

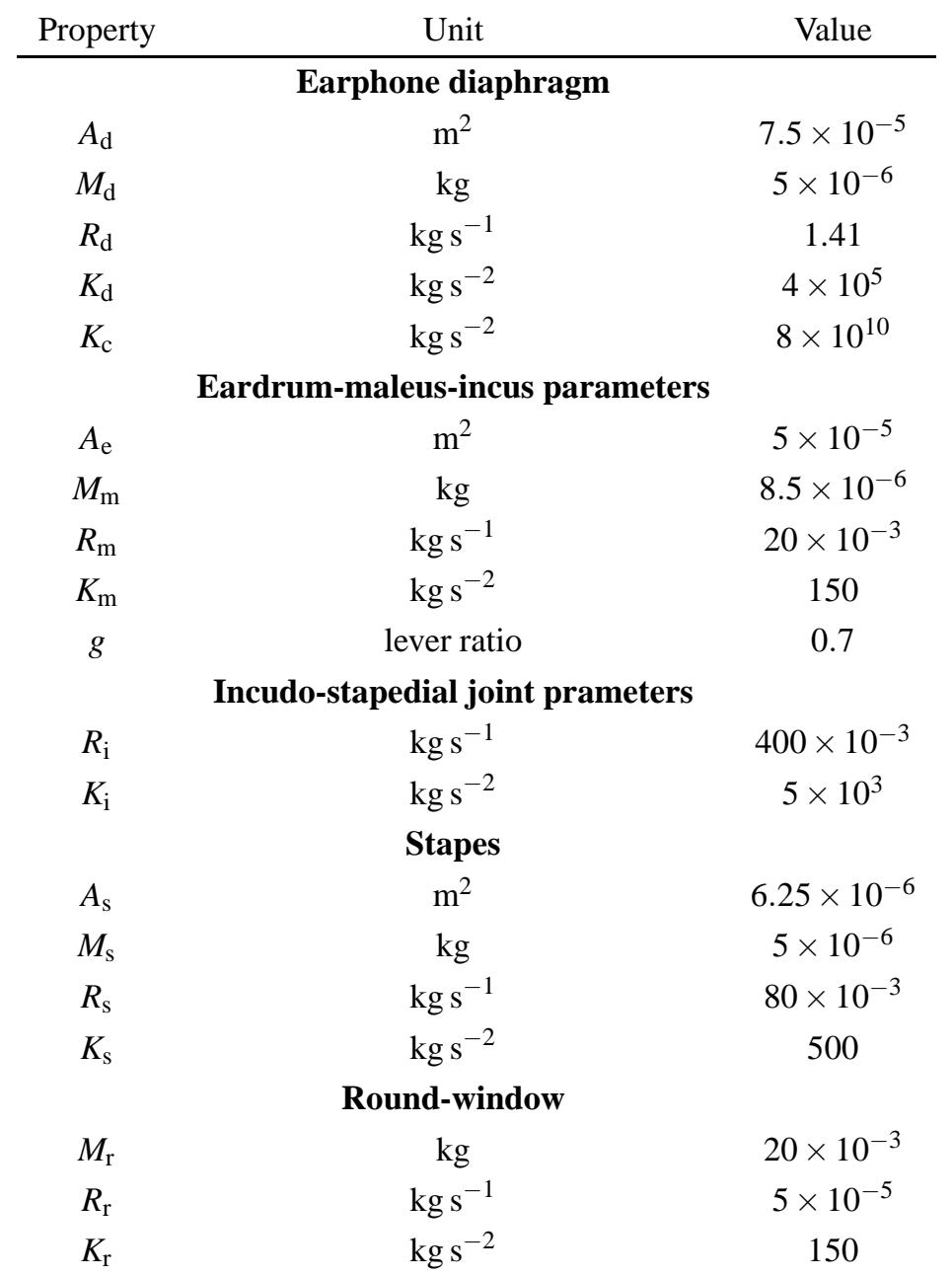


Table C.2: Parameter values for cochlear mechanics.

\begin{tabular}{ccccc} 
Property & Unit & Base & Middle & Appex \\
\hline$M$ & $\mathrm{~kg}$ & $2.8 \times 10^{-11}$ & $5 \times 10^{-10}$ & $2.8 \times 10^{-8}$ \\
$R$ & $\mathrm{~kg} \mathrm{~s}^{-1}$ & $9.4 \times 10^{-7}$ & $9.23 \times 10^{-7}$ & $1.85 \times 10^{-6}$ \\
$K$ & $\mathrm{~kg} \mathrm{~s}^{-2}$ & $1.96 \times 10^{-1}$ & $1.06 \times 10^{-2}$ & $7.64 \times 10^{-4}$ \\
$m$ & $\mathrm{~kg} \mathrm{~m}^{-2}$ & $1.24 \times 10^{-2}$ & $7.1 \times 10^{-2}$ & $4.1 \times 10^{-1}$ \\
$r$ & $\mathrm{~kg} \mathrm{~s}^{-1} \mathrm{~m}^{-2}$ & $4.8 \times 10^{2}$ & $7.97 \times 10^{2}$ & $1.68 \times 10^{3}$ \\
$k$ & $\mathrm{~kg} \mathrm{~s}^{-2} \mathrm{~m}^{-2}$ & $1.96 \times 10^{8}$ & $1 \times 10^{7}$ & $3.14 \times 10^{5}$ \\
$\alpha_{\mathrm{d}}$ & $\mathrm{Am}^{-1}$ & $1.6 \times 10^{-3}$ & $6.23 \times 10^{-4}$ & $2.04 \times 10^{-4}$ \\
$\alpha_{\mathrm{v}}$ & $\mathrm{Cm}^{-1}$ & $4.36 \times 10^{-6}$ & $1.78 \times 10^{-6}$ & $6.81 \times 10^{-7}$ \\
$I_{\mathrm{max}}$ & $\mathrm{pA}$ & 670 & 320 & 83 \\
$C_{\mathrm{g}}$ & $\mathrm{pF}$ & 18 & 33 & 70 \\
$T$ & $\mathrm{mC}^{-1}$ & $2.4 \times 10^{6}$ & $2.4 \times 10^{6}$ & $2.4 \times 10^{6}$ \\
$A$ & $\mathrm{~m}^{2}$ & $6.3 \times 10^{-6}$ & $1.4 \times 10^{-6}$ & $3.1 \times 10^{-3}$ \\
$w$ & $\mathrm{~m}$ & $3.1 \times 10^{-4}$ & $4 \times 10^{-4}$ & $5 \times 10^{-4}$ \\
$L$ & $\mathrm{~m}$ & & $3.5 \times 10^{-2}$ &
\end{tabular}

In Table. C.2, the parameter values are listed for the base, the middle of the cochlea $(L / 2)$, and the apex. Log-quadratic interpolation were used to interpolate intermediate values between these three locations.

\section{C.2 Electrical Parameter Values}

\section{C.2.1 Longitudinal Resistors}

Three longitudinal resistors $R_{8}, R_{9}$ and $R_{11}$ connect adjacent section of the electrical model (Fig. 3.7) of the organ of Corti. The parameter values of these resistors can be estimated by using the specific resistances (electrical resistivity) of perilymph and endolymph, the cross-sectional areas of the cochlear fluid spaces 
and the following relation for electrical resistivity (George, 2007, Chapter 3):

$$
R=\rho \frac{l}{A}
$$

where $R$ is here the longitudinal resistance, $\rho$ is the specific resistance of perilymph or endolymph, $l$ is the length (here $l=\Delta x$ ) and $A$ is the cross-sectional for each of three compartments of the cochlea (Fig. C.1).

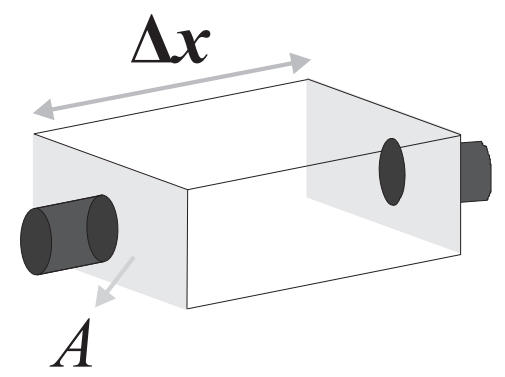

Figure C.1: Calculating parameter values for longitudinal resistors.

The longitudinal resistances have been estimated in Strelioff (1973) for the guinea pig by using the specific resistances of perilymph and endolymph reported in Misrahy et al. (1958) and the cross-sectional areas of each compartment of the guinea pig cochlea that were available that time. We recalculated these estimates using recent measurements of the cross-sectional areas of the guinea pig cochlea (Thorne et al., 1999) and the results are in reasonable agreement with the estimations of Strelioff (1973). The results of these comparisons is shown in Fig. C.2. A longitudinal average of these resistances was employed in Ramamoorthy et al. (2007).

The specific resistances of $0.719 \Omega \mathrm{m}$ for the perilymph and $0.598 \Omega \mathrm{m}$ for the endolymph of the guinea pig are reported in Misrahy et al. (1958) and these values have also been used for the human cochlea (Rattay et al., 2001; Tran et al., 2013). By using the cross-sectional area of the human cochlear fluid spaces measured in Wysocki (1999), the parameter values for longitudinal resistors are estimated and presented in Fig. C.3. 
(a)

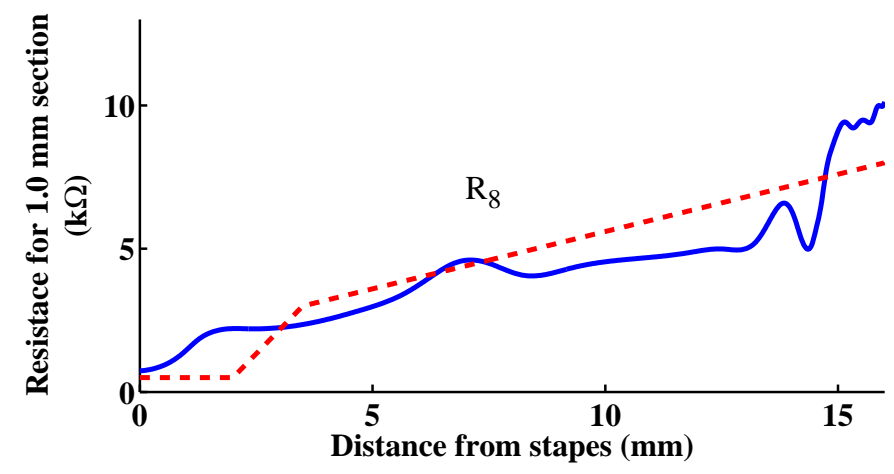

(b)

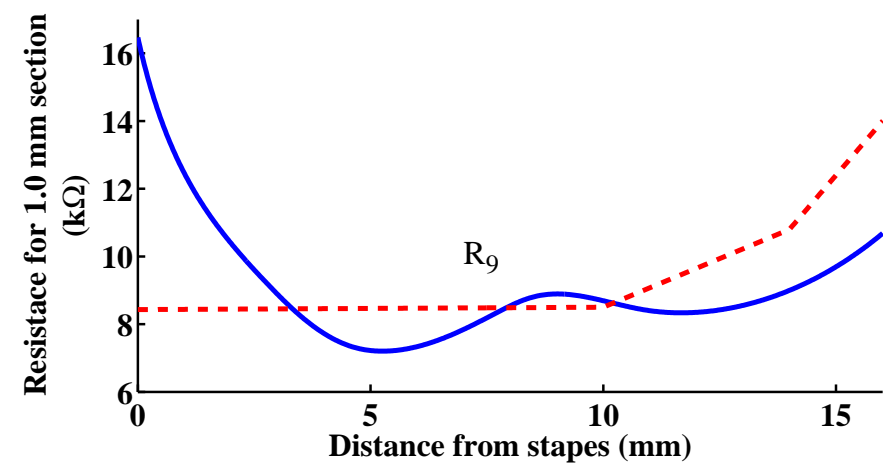

(c)

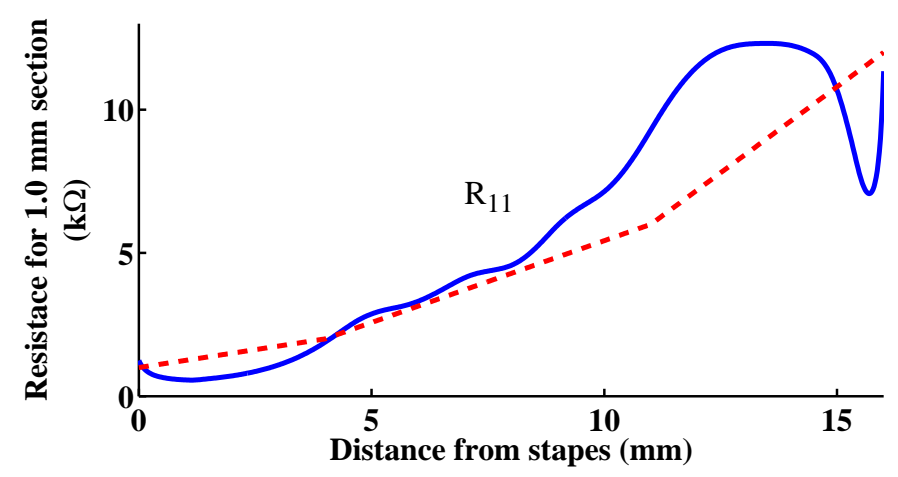

Figure C.2: Comparison between the estimates of longitudinal resistors reported in Strelioff (1973) and new estimates by using more recent measurements of the guinea pig cochlear fluid spaces (Thorne et al., 1999) and the specific resistances of the perilymph and the endolymph. The resistances along the scalae vestibuli, media and tympani are represented by $R_{8}, R_{9}$ and $R_{11}$, respectively. The red dashed lines show the estimates of Strelioff (1973). 
(a)

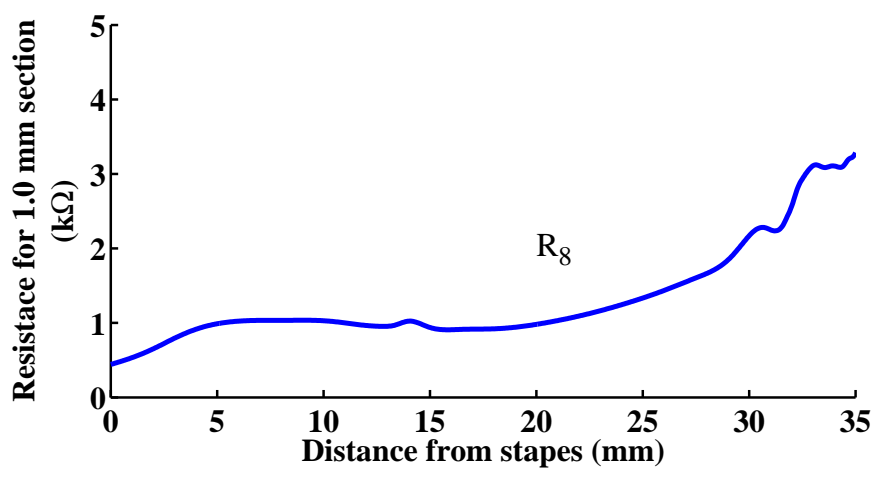

(b)

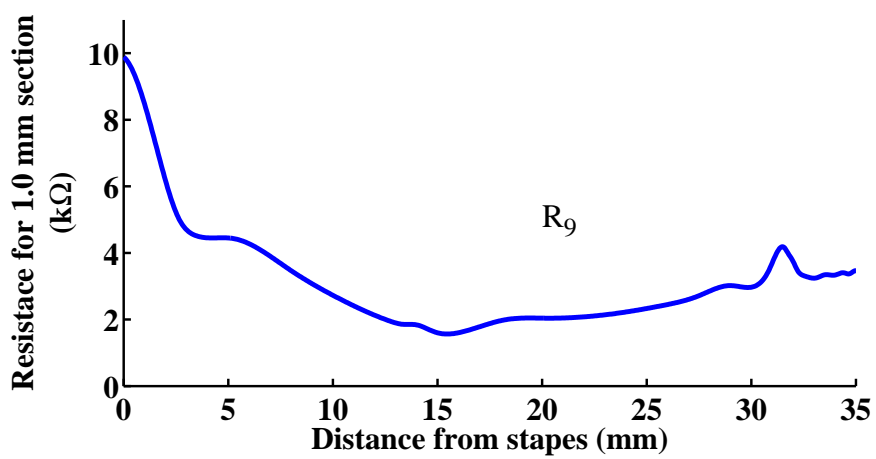

(c)

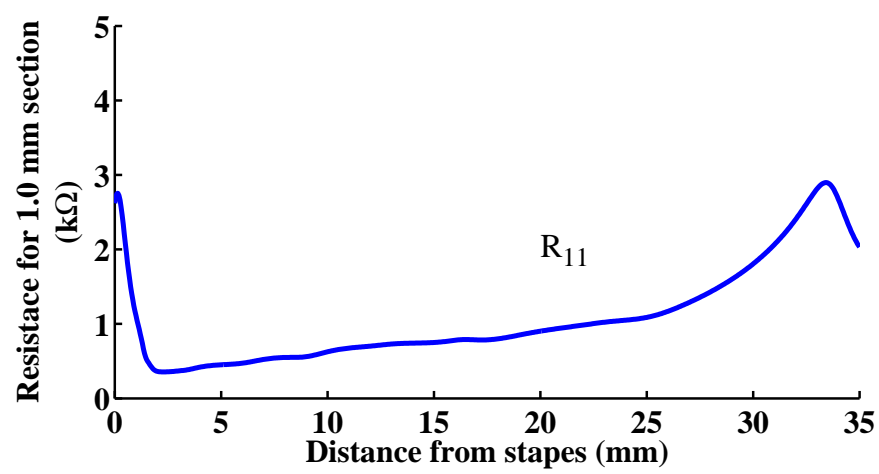

Figure C.3: The human cochlear fluid spaces (Wysocki, 1999) and the specific resistances of the perilymph and the endolymph were used to calculate the longitudinal resistors $R_{8}, R_{9}$ and $R_{11}$. 
It should be noted that in the human cochlea, Reissner's membrane which separates the scala media from the scala vestibuli is not accurately identifiable using imaging or other contemporary measuring techniques, and so a combination of these two compartments has been measured Wysocki (1999); Thorne et al. (1999). The ratio of endolymphatic cross sectional area to scala vestibuli cross sectional area is assumed to be the same in human as in guinea pig in Fig. C.3.

\section{C.2.2 Radial Electrical Elements}

In general, a passive cell membrane is modelled as a resistor and capacitor in parallel combination (Keener and Sneyd, 2009b, Chapter 8). However, due to the compartmentalisation of the organ of Corti, the membrane of the OHC can be divided into two independent membranes each of which can be modelled by a separate parallel combination of a resistor and a capacitor (Mistrík et al., 2009). The apical part (hair bundle) is modelled by $R_{3}, C_{3}$, and the basolateral part (soma) is modelled by $R_{4}, C_{4}$ in the proposed configuration (Fig. 3.7).

A cell membrane can be observed as two parallel conducting plates separated by an insulator, and therefore its capacitance is proportional to the area of the membrane (Keener and Sneyd, 2009b, Chapter 2). In mammals, the area of the OHC soma increases by a factor of $3-5$ from the base to the apex (Zajic and Schacht, 1987; He et al., 1994), consequently the parameter value of $C_{4}$ increases by the same factor (Housley and Ashmore, 1992). There are however only a very limited number of studies focused on measuring the dimensions of the hair bundle in mammalian species and human in particular (see Breneman et al. (2009); Johnson et al. (2011) and references within). Some measurements suggest that the ratio of the membrane area of $\mathrm{OHC}$ soma and the area of the hair bundle can be assumed to be constant along the cochlea length which results in a constant ratio of $C_{4}$ to $C_{3}$ (Johnson et al., 2011; Nam and Fettiplace, 2012).

Recent measurements and estimations of the $\mathrm{OHC}$ indicate that the parameter value of $R_{4}$ increases by approximately 6-fold from the base to the apex (Fig.6 D of Johnson et al. (2011)) which is less (approximately 50-fold) than what has been 
previously reported (Housley and Ashmore, 1992; Mammano and Ashmore, 1996; Mistrík et al., 2009).

The OHC total membrane capacitance $\left(C_{\mathrm{m}}=C_{3}+C_{4}\right)$ and total membrane conductance (at resting potential) $G_{\mathrm{r}}=1 / R_{3}+1 / R_{4}$ determine the membrane time constant of the $\mathrm{OHC}\left(\tau_{\mathrm{OHC}}=C_{\mathrm{m}} / G_{\mathrm{r}}\right)$ (Ashmore, 2011; Johnson et al., 2011). Prior to the recent measurements and estimations of the membrane time constant the OHC (Johnson et al., 2011), the cochlea amplification was thought to be restricted by this time constant for higher frequency stimuli. The recent measurement reveals that the $\mathrm{OHC}$ membrane time constant due to the notable difference between ion concentration in vivo and in vitro, was overestimated by a factor of 10 (Johnson et al., 2011; Nam and Fettiplace, 2012) (also see Section 2.4).

It is noteworthy that the measurements and estimates of Johnson et al. (2011) imply that prestin can provide amplification over the full frequency range of mammalian hearing. However, the current technology prevents stimulation very small and fragile OHCs at the base and measurement of them. Therefore, precise and direct measurements have yet to be conducted to settle the time constant issue (Ashmore, 2011).

The parameter values of the radial electrical elements of the electrical coupling of the model for the base and the apex are listed in Table. C. 3 based on the aforementioned measurements and model estimates. The particular parameters having the most uncertainty due to limited availability of data are $R_{1}, R_{2}, R_{5}$ and $R_{6}$. The parameter values between these two locations are assumed to be logarithmically spaced.

$\tau_{\mathrm{OHC}}$ of the model is compared with the data of (Johnson et al., 2011) in Fig. C.4. 


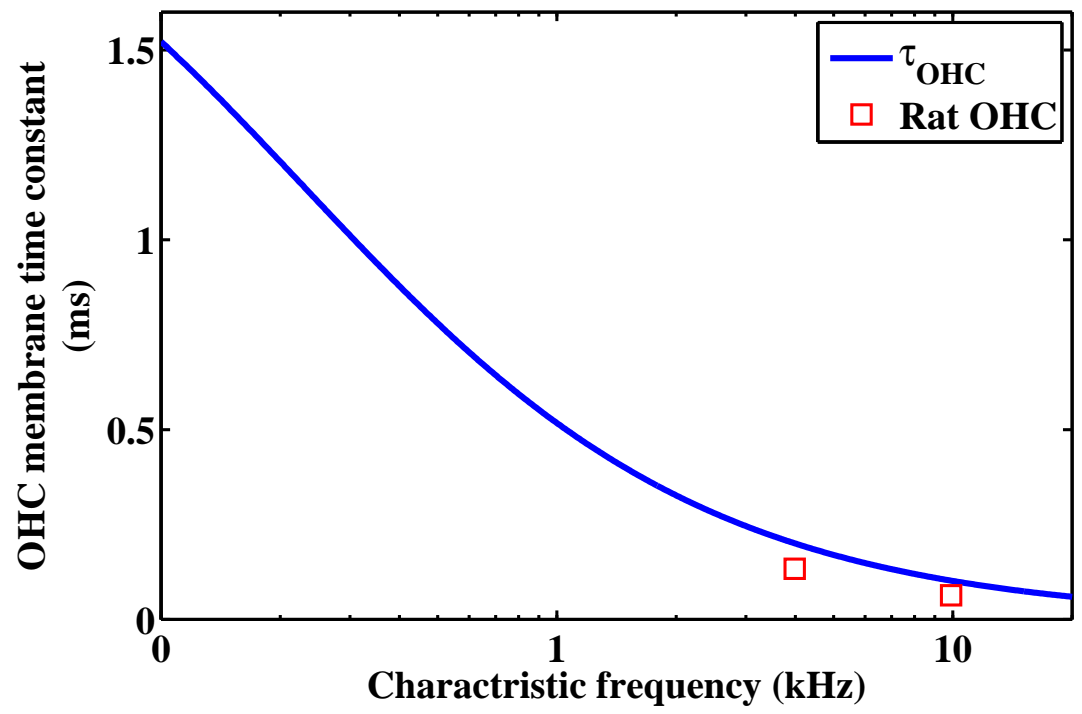

Figure C.4: $\tau_{O H C}$ is calculated by using the parameter values of Table. C.3. The membrane time constants for the rat $\mathrm{OHC}$ have been extracted from part C of Fig 7 of Johnson et al. (2011). 
Table C.3: Electrical parameters used in the model.

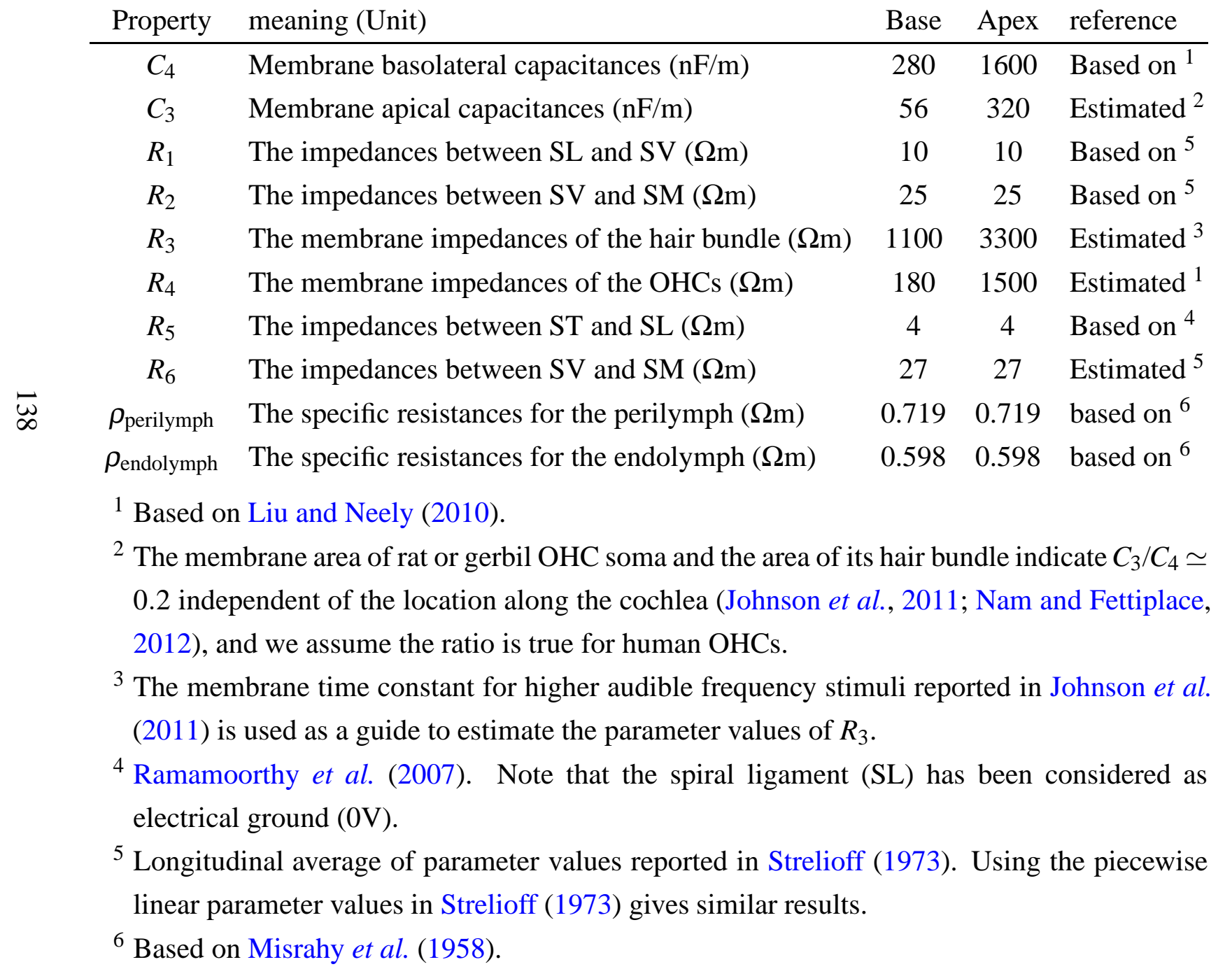




\section{C.3 Calibration}

The stimulus force on the earphone diaphragm $f(t)$ determines the level of sound pressure (see Fig. 3.6 (A)). The model was calibrated by considering the displacement of the BM in the human cochlea. Fig. C.5 shows the results of the calibration. These results are consistent with the modelling results of the human BM displacement reported in Nobili et al. (2003).

The nonlinearity in MET current is responsible for the intensity-dependent
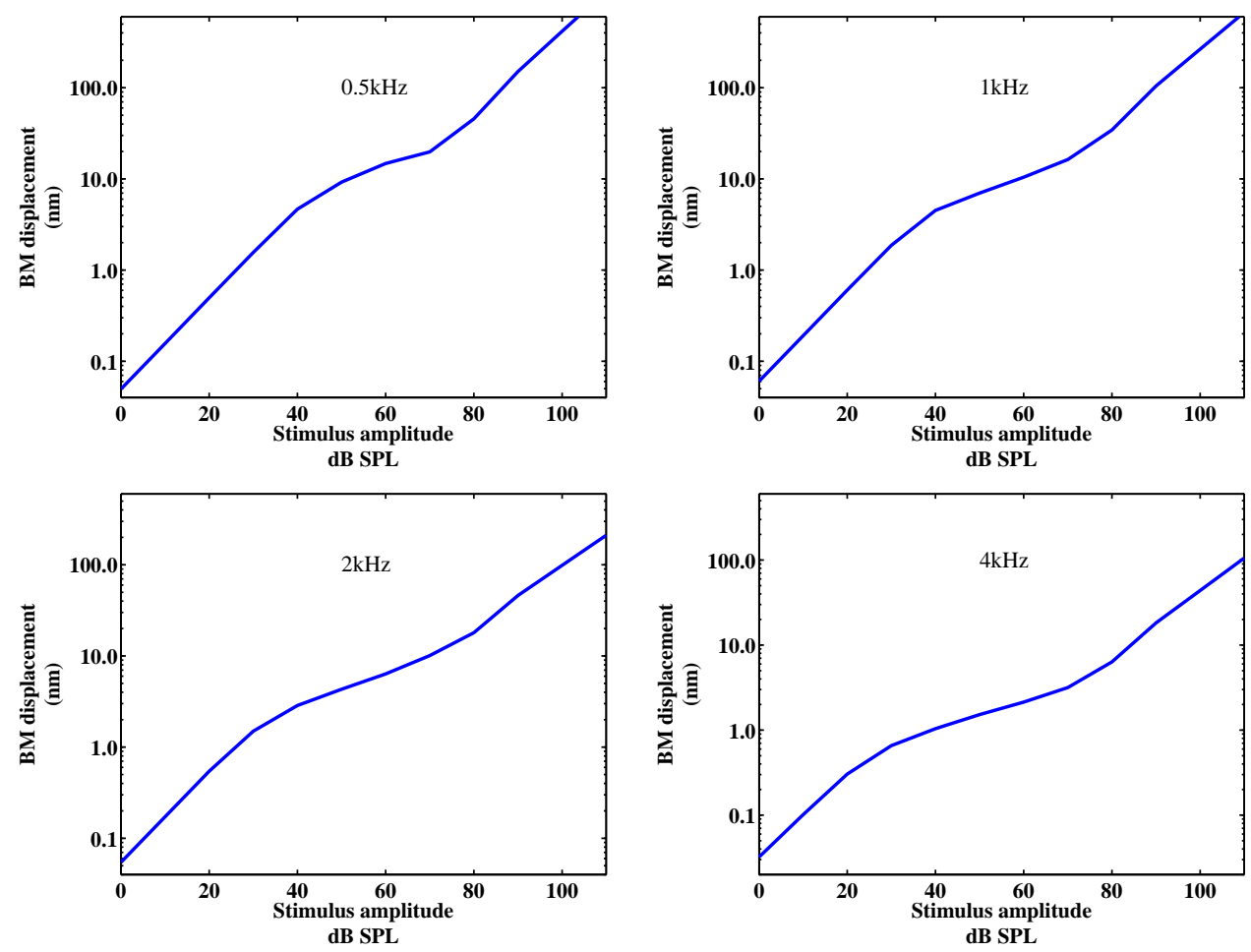

Figure C.5: The BM displacement responses grow nonlinearly with the stimulus intensity at the indicated characteristic frequencies.

nonlinear growth of responses. 


\section{Bibliography}

Adrian, E. D. (1931). "The microphonic action of the cochlea: An interpretation of Wever and Brays experiments", Proceedings of the Physiological Society 71, XXViii-XXiX. 3, 24

Ashmore, J. (2008). "Cochlear outer hair cell motility", Physiological Reviews 88, 173-210. 12, 14, 22, 79

Ashmore, J. (2011). "Pushing the envelope of sound", Neuron 70, 1021-1022. 21, 136

Atcherson, S. R. and Stoody, T. M. (2012). Auditory Electrophysiology: A Clinical Guide (Thieme). 4

Ayat, M. and Teal, D. P. (????). "Model based prediction of the existence of the spontaneous cochlear microphonic", in Mechanics of Hearing, Attica, Greece, July 2014. 8

Ayat, M., Teal, D. P., and McGuinness, M. (2014). "An integrated model for the cochlear microphonic", Biocybernetics and Biomedical Engineering . 8

Ayat, M., Teal, D. P., Searchfield, G. D., and Razali, N. (????). "Cochlear microphonic broad tuning curves", in Mechanics of Hearing, Attica, Greece, July 2014. 8

Ayat, M. and Teal, P. D. (2013a). "Modelling the generation of the cochlear microphonic", in Engineering in Medicine and Biology Society (EMBC), 2013 35th Annual International Conference of the IEEE, 7168-7171 (IEEE). 8 
Ayat, M. and Teal, P. D. (2013b). "Using circuit analogies for analysis of cochlear models", Biomedical Engineering Letters 3, 263-272. 8

Békésy, G. (1951). "DC potentials and energy balance of the cochlear partition", The Journal of the Acoustical Society of America 23, 576-582. 24

Bellizzi, S. and Bouc, R. (2007). “An amplitude-phase formulation for nonlinear modes and limit cycles through invariant manifolds", Journal of sound and vibration 300, 896-915. 96

Berlin, C. I., Hood, L., Morlet, T., Rose, K., and Brashears, S. (2003). “Auditory neuropathy/dys-synchrony: Diagnosis and management", Mental retardation and developmental disabilities research reviews 9, 225-231. 26

Bertaccini, D. and Sisto, R. (2011). "Fast numerical solution of nonlinear nonlocal cochlear models", Journal of computational physics 230, 2575-2587. 51, 65, 66,119

Borg, E., Canlon, B., and Engström, B. (1995). "Noise-induced hearing loss. Literature review and experiments in rabbits. Morphological and electrophysiological features, exposure parameters and temporal factors, variability and interactions", Scand. Audiol. Suppl. 40, 1-147. 108

Braun, M. (2013). "High-multiple spontaneous otoacoustic emissions confirm theory of local tuned oscillators", SpringerPlus 2, 135. 20, 88, 99

Breneman, K. D., Brownell, W. E., and Rabbitt, R. D. (2009). "Hair cell bundles: flexoelectric motors of the inner ear", PLoS One 4, e5201. 135

Bright, K. E. (2002). “Spontaneous otoacoustic emissions", MS Robinette \& TJ Glattke (eds). Otoacoustic Emissions: Clinical Applications 74-94. 19

Bright, K. E. (2007). "Spontaneous otoacoustic emissions in populations with normal hearing sensitivity", in Otoacoustic emissions: clinical applications, edited by M. S. Robinette and T. J. Glattke, chapter 3 (Thieme). 91 
Buus, S. and Florentine, M. (2002). "Growth of loudness in listeners with cochlear hearing losses: recruitment reconsidered", JARO-Journal of the Association for Research in Otolaryngology 3, 120-139. 61

Cai, H., Manoussaki, D., and Chadwick, R. (2005). "Effects of coiling on the micromechanics of the mammalian cochlea", Journal of The Royal Society Interface 2, 341-348. 31

Ceresa, M., Perez, F., Vera, S., Carranza, N., Jover, J. H., Mistrik, P., and Gonzalez Ballester, M. A. (2013). "Functional simulation of the cochlea for implant optimization", in Engineering in Medicine and Biology Society (EMBC), 2013 35th Annual International Conference of the IEEE, 4541-4544 (IEEE). 33

Cha, P. D., Rosenberg, J. J., and Dym, C. L. (2000). Fundamentals of modeling and analyzing engineering systems (Cambridge University Press). 52

Chadwick, R. (1997). "What should be the goals of cochlear modeling?", The Journal of the Acoustical Society of America 102, 3054. 87

Cheatham, M., Naik, K., and Dallos, P. (2011). "Using the cochlear microphonic as a tool to evaluate cochlear function in mouse models of hearing", JAROJournal of the Association for Research in Otolaryngology 12, 113-125. 3, 4, 23, 39, 40, 46, 67, 68, 104, 105, 108

Chen, H.-h. A. and Narins, P. (2012). "Wind turbines and ghost stories: the effects of infrasound on the human auditory system", Acoustics Today 8, 51-56. 4, 25

Cheng, L., White, R. D., and Grosh, K. (2008). "Three-dimensional viscous finite element formulation for acoustic fluid-structure interaction", Computer methods in applied mechanics and engineering 197, 4160-4172. 33

Chertoff, M. E., Earl, B. R., Diaz, F. J., and Sorensen, J. L. (2012). "Analysis of the cochlear microphonic to a low-frequency tone embedded in filtered noise", 
The Journal of the Acoustical Society of America 132, 3351-3362. 4, 27, 77, 108

Choi, Y.-S., Lee, S.-Y., Parham, K., Neely, S. T., and Kim, D. O. (2008). "Stimulus-frequency otoacoustic emission: Measurements in humans and simulations with an active cochlear model", The Journal of the Acoustical Society of America 123, 2651. 63

Clark, W., Kim, D., Zurek, P., and Bohne, B. (1984). "Spontaneous otoacoustic emissions in chinchilla ear canals: correlation with histopathology and suppression by external tones", Hearing research 16, 299-314. 91

Dallos, P. (1983). "Some electrical circuit properties of the organ of Corti. i. analysis without reactive elements", Hearing Research 12, 89-119. 25, 36, 39, 46, 109

Dallos, P. (1984). "Some electrical circuit properties of the organ of Corti. ii. analysis including reactive elements", Hearing Research 14, 281-291. 36, 39, 46

Dallos, P. (1992). “The active cochlea", The journal of neuroscience 2, 4575-4585. 66

Dallos, P., Billone, N., Durrant, J., Wang, C.-Y., and Raynor, S. (1972). "Cochlear inner and outer hair cells: functional differences”, Science 177, 356-358. 17

Dallos, P., Cheatham, M. A., and Ferraro, J. (2005). "Cochlear mechanics, nonlinearities, and cochlear potentials", The Journal of the Acoustical Society of America 55, 597-605. 6, 73, 74, 98

Dallos, P. and Evans, B. N. (1995). "High-frequency motility of outer hair cells and the cochlear amplifier", Science 267, 2006-2009. 39

Darlene, R., MARGARET, W. S., GE WANG, P., MICHAEL, W. V., GEORGE, A. G., and J GAIL, N. (1998). "In vivo measures of cochlear length and 
insertion depth of nucleus cochlear implant electrode arrays", Ann Otol Rhinol Laryngol 107, 1B11. 58

Davis, H. (1965). "A model for transducer action in the cochlea", Cold Spring Harbor symposia on quantitative biology 30, 181-190. 36, 37

De Boer, E. (1996). "Mechanics of the cochlea: modeling efforts", in The cochlea, edited by P. Dallos, A. Popper, and R. Fay, 258-317 (Springer). 103

Dorf, R. C. and Bishop, R. H. (2011). Modern control systems (Pearson). 89

Duifhuis, H. (2012). Cochlear Mechanics, SpringerLink : Bücher (Springer). 29, 129

Duke, T. (2002). “The power of hearing”, Physics world 15, 29-33. 2, 3

Elliott, S., Ku, E., and Lineton, B. (2007). "A state space model for cochlear mechanics", The Journal of the Acoustical Society of America 122, 2759. 6, $34,40,51,52,65,88,91,92,94,96,101,106$

Erixon, E., Högstorp, H., Wadin, K., and Rask-Andersen, H. (2009). "Variational anatomy of the human cochlea: Implications for cochlear implantation", Otology \& Neurotology 30, 14-29. 11

Fettiplace, R. (2006). "Active hair bundle movements in auditory hair cells", The Journal of physiology 576, 29-36. 21

Fettiplace, R. and Hackney, C. M. (2006). "The sensory and motor roles of auditory hair cells", Nature Reviews Neuroscience 7, 19-29. 10, 13, 16, 21, 36

Fletcher, H. (1938). "The mechanism of hearing as revealed through experiment on the masking effect of thermal noise", Proceedings of the National Academy of Sciences of the United States of America 24, 265-274. 16 
Fridberger, A., de Monvel, J. B., Zheng, J., Hu, N., Zou, Y., Ren, T., and Nuttall, A. (2004). "Organ of corti potentials and the motion of the basilar membrane", The Journal of neuroscience 24, 10057-10063. 63, 73, 74, 105

Frolenkov, G. I. (2006). "Regulation of electromotility in the cochlear outer hair cell”, The Journal of physiology 576, 43-48. 14

Furness, D. N. and Hackney, C. M. (2008). "Molecular anatomy of receptor cells and organ of corti", in The senses: a comprehensive reference, volume 3:Audition, 108-131 (Elsevier, Canada). 11

Geisler, C. D. and Cai, Y. (1996). "Relationships between frequency-tuning and spatial-tuning curves in the mammalian cochlea", The Journal of the Acoustical Society of America 99, 1550. 77

Gelfand, S. A. (2010). Hearing: An Introduction to Psychological and Physiological Acoustics (Informa Healthcare). 6, 9, 10, 11, 13, 17, 18, 19, 22, 24, 25, 31, 61, 74, 105

George, G. S. (2007). Engineering Physics (Technical Publications). 132

Gold, T. (1948). "Hearing. ii. the physical basis of the action of the cochlea", Proceedings of the Royal Society of London. Series B-Biological Sciences 135, 492-498. 30

Greenwood, D. (1990a). "A cochlear frequency-position function for several species-29 years later", The Journal of the Acoustical Society of America 87, 2592-2605. 78

Greenwood, D. D. (1990b). "A cochlear frequency-position function for several species29 years later", The Journal of the Acoustical Society of America 87, 2592. 58, 67, 129, 130

Guinan Jr, J. J., Salt, A., and Cheatham, M. A. (2012). "Progress in cochlear physiology after békésy", Hearing research 293, 12-20. 20, 30, 39 
Hall, J. (2000). Handbook of otoacoustic emissions, chapter 3 (Singular Publishing Group, San Diego). 20, 96, 99

Hamilton, T. J., Jin, C., van Schaik, A., and Tapson, J. (2008). "An active 2-d silicon cochlea", Biomedical Circuits and Systems, IEEE Transactions on 2 , 30-43. 128

Harris, J. and Salt, A. (2008). "Meniere's disease", in The senses: a comprehensive reference, volume 3:Audition, 157-163 (Elsevier, Canada). 25, 26

He, D. Z., Evans, B. N., and Dallos, P. (1994). "First appearance and development of electromotility in neonatal gerbil outer hair cells", Hearing research 78, 7790. 135

He, W., Porsov, E., Kemp, D., Nuttall, A., and Ren, T. (2012a). "The group delay and suppression pattern of the cochlear microphonic potential recorded at the round window", PloS one 7, e34356. 6, 74, 105, 108

He, W., Porsov, E., Kemp, D., Nuttall, A. L., and Ren, T. (2012b). "The group delay and suppression pattern of the cochlear microphonic potential recorded at the round window", PLoS One 7, e34356. 24

He, W. and Ren, T. (2013). "Basilar membrane vibration is not involved in the reverse propagation of otoacoustic emissions", Scientific reports 3. 4, 98

Holley, M. C. (1996). "Outer hair cell motility", in The cochlea, edited by P. Dallos, A. Popper, and R. Fay, 386-434 (Springer). 34

Honrubia, V. and Ward, P. H. (1968). "Longitudinal distribution of the cochlear microphonics inside the cochlear duct (guinea pig)", The Journal of the Acoustical Society of America 44, 951-958. 4, 6, 24, 39, 63, 73, 75, 98

Housley, G. and Ashmore, J. (1992). "Ionic currents of outer hair cells isolated from the guinea-pig cochlea.”, The Journal of physiology 448, 73-98. 135, 136 
$\mathrm{Hu}$, B. (2012). "Noise-induced structural damage to the cochlea", in NoiseInduced Hearing Loss, 57-86 (Springer). 108

Hu, T., Thibodeau, T., and Teel, A. R. (2009). "Analysis of oscillation and stability for systems with piecewise linear components via saturation functions", in American Control Conference, 2009. ACC'09., 1911-1916 (IEEE). 96

Iurato, S. (1962). "Functional implications of the nature and submicroscopic structure of the tectorial and basilar membranes", The Journal of the Acoustical Society of America 34, 1386-1395. 31

Iwasa, K. H. and Sul, B. (2008). "Effect of the cochlear microphonic on the limiting frequency of the mammalian ear", The Journal of the Acoustical Society of America 124, 1607. 39

Johnson, S. L., Beurg, M., Marcotti, W., and Fettiplace, R. (2011). "Prestindriven cochlear amplification is not limited by the outer hair cell membrane time constant", Neuron, Elsevier 70, 1143-1154. 21, 37, 39, 135, 136, 137, 138

Kandel, E. R., Schwartz, J. H., Jessell, T. M., Siegelbaum, S. A., and Hudspeth, A. J. (2012). Principles of neural science, fifth edition (McGraw-Hill New York). 1, 9, 12, 15

Keener, J. and Sneyd, J. (2009a). Mathematical Physiology : Systems Physiology, volume 2 (Springer Verlag). 33

Keener, J. and Sneyd, J. (2009b). Mathematical Physiology : Systems Physiology, volume 1 (Springer Verlag). 135

Kemp, D. (1979). "Evidence of mechanical nonlinearity and frequency selective wave amplification in the cochlea", Archives of oto-rhino-laryngology 224, 3745. 6 
Kemp, D. T. (1978a). "Stimulated acoustic emissions from within the human auditory system", The Journal of the Acoustical Society of America 64, 13861391. 18

Kemp, D. T. (1978b). "Stimulated acoustic emissions from within the human auditory system", The Journal of the Acoustical Society of America 64, 1386. 69

Kennedy, H., Crawford, A., and Fettiplace, R. (2005). "Force generation by mammalian hair bundles supports a role in cochlear amplification", Nature 433, 880-883. 21

Khalil, H. (2001). Nonlinear System, 3rd edition (Prentice-Hall). 96

Khoo, M. C. (2000). Physiological control systems (IEEE press). 52, 96

Killion, M. and Niquette, P. (2000). "What can the pure-tone audiogram tell us about a patients SNR loss", Hear J 53, 46-53. 109

Kim, D. J., Mountain, D. C., and Hubbard, A. E. (2011). "How much do somatic and hair bundle motility contribute to cochlear amplification?", in AIP Conference Proceedings, volume 1403, 632. 120

Kletsky, E. J. and Zwislocki, J. J. (1979). "Cochlear-microphonic versus hair-cell tuning in the cochlea", The Journal of the Acoustical Society of America $\mathbf{6 5}$, S84-S84. 77

Kletsky, E. J. and Zwislocki, J. J. (1980). "CM tuning can be compatible with sharply tuned receptor potentials", Hearing research 2, 549-557. 74

Kohllöffel, L. (1990). "Cochlear mechanics: coiling effects (i, ii) and the absorption equation (iii)”, Hearing research 49, 19-27. 31

Kolston, P. J. (2000). "Finite-element modelling: a new tool for the biologist", Philosophical Transactions of the Royal Society of London. Series A: Mathematical, Physical and Engineering Sciences 358, 611-631. 109 
$\mathrm{Ku}$, E., Elliott, S., and Lineton, B. (2009). "Limit cycle oscillations in a nonlinear state space model of the human cochlea", The Journal of the Acoustical Society of America 126, 739-750. 96

Ku, E. M. (2008). "Modelling the human cochlea", Ph.D. thesis, University of Southampton. 15, 34, 58, 79, 88, 91, 92, 94

Lagarde, M. M. M., Drexl, M., Lukashkina, V. A., Lukashkin, A. N., and Russell, I. J. (2008). "Outer hair cell somatic, not hair bundle, motility is the basis of the cochlear amplifier", Nature neuroscience 11, 746-748. 21, 107

Leake, P. A., Hradek, G. T., Snyder, R. L., et al. (2006). "Neonatal deafness results in degraded topographic specificity of auditory nerve projections to the cochlear nucleus in cats", Journal of Comparative Neurology 497, 13-31. 27

Leventhall, G. (2006). "Infrasound from wind turbines-fact, fiction or deception", Canadian acoustics 34, 29-36. 25

Leventhall, G. (2007). "What is infrasound?", Progress in biophysics and molecular biology 93, 130-137. 25

Liberman, M. C. (1982). "The cochlear frequency map for the cat: Labeling auditory-nerve fibers of known characteristic frequency", The Journal of the Acoustical Society of America 72, 1441-1449. 16, 129

Liberman, M. C., Gao, J., He, D. Z., Wu, X., Jia, S., and Zuo, J. (2002). "Prestin is required for electromotility of the outer hair cell and for the cochlear amplifier", Nature 419, 300-304. 4, 21, 24, 67, 68, 69, 105

Liu, Y.-W. and Neely, S. T. (2009). "Outer hair cell electromechanical properties in a nonlinear piezoelectric model", The Journal of the Acoustical Society of America 126, 751. 22, 41, 42, 45, 130

Liu, Y.-W. and Neely, S. T. (2010). "Distortion product emissions from a cochlear model with nonlinear mechanoelectrical transduction in outer hair cells", The 
Journal of the Acoustical Society of America 127, 2420-2432. vi, 37, 40, 41, 42, 44, 45, 46, 49, 53, 58, 130, 138

Lonsbury-Martin, B. and Martin, G. (1988). "Incidence of spontaneous otoacoustic emissions in macaque monkeys: a replication", Hearing research 34, 313-317. 91

Lu, T. K., Zhak, S., Dallos, P., and Sarpeshkar, R. (2006). "Fast cochlear amplification with slow outer hair cells", Hearing research 214, 45-67. 120

Mammano, F. and Ashmore, J. F. (1996). "Differential expression of outer hair cell potassium currents in the isolated cochlea of the guinea-pig.", The Journal of physiology 496, 639-646. 136

Manley, G., Fay, R., and Popper, A., eds. (2008). Active Processes and Otoacoustic Emissions in Hearing (Springer). 17, 18, 19

Manley, G. A. and van Dijk, P. (2007). "Otoacoustic emissions in amphibians, lepidosaurs, and archosaurs", in Active Processes and Otoacoustic Emissions in Hearing, 211-260 (Springer). 26

Maoiléidigh, D. Ó. and Hudspeth, A. (2013). "Effects of cochlear loading on the motility of active outer hair cells", Proceedings of the National Academy of Sciences 110, 5474-5479. 21, 129

Martin, P. (2007). "Active hair-bundle motility of the hair cells of vestibular and auditory organs", in Active Processes and Otoacoustic Emissions in Hearing, 93-143 (Springer). 12

Masood, A., Teal, P., and Hollitt, C. (2012). "A non-invasive cochlear microphonic measurement system", Medical Engineering \& Physics 34, 11911195. 4, 24, 101

Meaud, J. and Grosh, K. (2010). "The effect of tectorial membrane and basilar membrane longitudinal coupling in cochlear mechanics", The Journal of the Acoustical Society of America 127, 1411-1421. 107 
Meddis, R. and Lopez-Poveda, E. A. (2010). "Overview", in Computational models of the auditory system, edited by R. Meddis, E. A. Lopez-Poveda, R. R. Fay, and A. N. Popper, volume 35 of Springer Handbook of Auditory Research, chapter 1 (Springer). 3

Miranker, W. L. and Miranker, A. (2002). Numerical Methods for Stiff Equations and Singular Perturbation Problems, volume 5 (Springer). 119

Misrahy, G. A., Hildreth, K. M., Shinabarger, E. W., and Gannon, W. J. (1958). "Electrical properties of wall of endolymphatic space of the cochlea (guinea pig)", American Journal of Physiology-Legacy Content 194, 396-402. 79, 132, 138

Mistrík, P., Mullaley, C., Mammano, F., and Ashmore, J. (2009). "Threedimensional current flow in a large-scale model of the cochlea and the mechanism of amplification of sound", Journal of The Royal Society Interface 6, 279-291. 16, 39, 40, 46, 48, 77, 135, 136

Moleti, A., Paternoster, N., Bertaccini, D., Sisto, R., and Sanjust, F. (2009). "Otoacoustic emissions in time-domain solutions of nonlinear non-local cochlear models", The Journal of the Acoustical Society of America 126, 2425. 51

Moller, A. R. (2006). Hearing: anatomy, physiology, and disorders of the auditory system (Academic Press). 1, 2, 14, 20

Moreno, I. and Suárez, R. (2004). "Existence of periodic orbits of stable saturated systems", Systems \& control letters 51, 293-309. 96

Mountain, D. C. and Hubbard, A. E. (1994). "A piezoelectric model of outer hair cell function", The Journal of the Acoustical Society of America 95, 350. 22, 45

Müller, U. and Gillespie, P. (2008). "Silencing the cochlear amplifier by immobilizing prestin", Neuron 58, 299-301. 60 
Naidu, R. C. and Mountain, D. C. (2001). "Longitudinal coupling in the basilar membrane", JARO-Journal of the Association for Research in Otolaryngology 2, 257-267. 107

Nam, J.-H. and Fettiplace, R. (2010). "Force transmission in the organ of corti micromachine", Biophysical journal 98, 2813-2821. 21, 22, 37, 40

Nam, J.-H. and Fettiplace, R. (2012). "Optimal electrical properties of outer hair cells ensure cochlear amplification", PloS one 7, e50572. 21, 22, 36, 37, 40, $135,136,138$

Neely, S. T. (1977). "Mathematical models of the mechanics of the cochlea", Ph.D. thesis, California Institute of Technology. 33

Neely, S. T. (1985). "Mathematical modeling of cochlear mechanics", The Journal of the Acoustical Society of America 78, 345. 44

Neely, S. T. (1993). "A model of cochlear mechanics with outer hair cell motility", The journal of the acoustical society of America 94, 137. 120

Neely, S. T. and Kim, D. O. (1986). "A model for active elements in cochlear biomechanics", The Journal of the Acoustical Society of America 79, 1472-80. $34,35,40,60,62,75$

Ni, G. (2012). "Fluid coupling and waves in the cochlea", Ph.D. thesis, University of Southampton. 51

Ni, G., Elliott, S., Ayat, M., and Teal, P. D. (2014). "Modelling cochlear mechanics", BioMed Research International 2014. 8

Ni, G., Elliott, S., Lineton, B., and Saba, R. (2011). "Finite element modelling of fluid coupling in the coiled cochlea", in AIP Conference Proceedings, volume $1403,350.31$

Nin, F., Hibino, H., Murakami, S., Suzuki, T., Hisa, Y., and Kurachi, Y. (2012a). "Computational model of a circulation current that controls electrochemical 
properties in the mammalian cochlea", Proceedings of the National Academy of Sciences 109, 9191-9196. 104

Nin, F., Reichenbach, T., Fisher, J. A., and Hudspeth, A. (2012b). "Contribution of active hair-bundle motility to nonlinear amplification in the mammalian cochlea", Proceedings of the National Academy of Sciences 109, 21076-21080. 107

Nobili, R., Mammano, F., and Ashmore, J. (1998). "How well do we understand the cochlea?", Trends in neurosciences 21, 159-167. 32

Nobili, R., Vetešnik, A., Turicchia, L., and Mammano, F. (2003). "Otoacoustic emissions from residual oscillations of the cochlear basilar membrane in a human ear model", Journal of the Association for Research in Otolaryngology 4, 478-494. 88, 92, 101, 106, 139

Norman, N. G., Shea Jr, J. J., and Orchik, D. J. (1997). "Cochlear microphonics in meniere's disease”, Otology \& Neurotology 18, 58-66. 4, 26

O'Beirne, G. A. (2005). "Mathematical modelling and electrophysiological monitoring of the regulation of cochlear amplification", Ph.D. thesis, University of Western Australia. Physiology. 38

O’Beirne, G. A. and Patuzzi, R. (2007). "Mathematical model of outer hair cell regulation including ion transport and cell motility", Hearing Research Elsevier 234, 29-51. 38

Oghalai, J. S. (2004). "The cochlear amplifier: augmentation of the traveling wave within the inner ear", Current opinion in otolaryngology \& head and neck surgery 12, 431. 21, 34

Osborne, M., Comis, S., and Pickles, J. O. (1988). "Further observations on the fine structure of tip links between stereocilia of the guinea pig cochlea", Hearing research 35, 99-108. 18 
Oxenham, A. J. and Shera, C. A. (2003). "Estimates of human cochlear tuning at low levels using forward and simultaneous masking", Journal of the Association for Research in Otolaryngology 4, 541-554. 61

Patuzzi, R. (1987). "A model of the generation of the cochlear microphonic with nonlinear hair cell transduction and nonlinear basilar membrane mechanics", Hearing Research 30, 73-82. 6, 39, 73, 74, 105

Patuzzi, R. (1996). "Cochlear micromechanics and macromechanics", in The cochlea, edited by P. Dallos, A. Popper, and R. Fay, 186-257 (Springer). 31, $34,35,43$

Patuzzi, R. (2011). "Ion flow in cochlear hair cells and the regulation of hearing sensitivity", Hearing research 280, 3-20. 35

Patuzzi, R. and Moleirinho, A. (1998). "Automatic monitoring of mechanoelectrical transduction in the guinea pig cochlea", Hearing research 125, 1-16. $4,25,26$

Patuzzi, R., Yates, G., and Johnstone, B. (1989). "Outer hair cell receptor current and sensorineural hearing loss", Hearing research 42, 47-72. 4

Patuzzi, R. B. and O’Beirne, G. A. (1999). "Boltzmann analysis of cm waveforms using virtual instrument software", Hearing research 133, 155-159. 4, 25, 26

Peng, A. W. and Ricci, A. J. (2011). "Somatic motility and hair bundle mechanics, are both necessary for cochlear amplification?”, Hearing research 273, 109-122. 21

Pickles, J. O. (2008). An introduction to the Physiology of Hearing, third edition (Emerald Group Publishing). 11, 14, 23

Pickles, J. O., Comis, S. D., and Osborne, M. (1984). "Cross-links between stereocilia in the guinea pig organ of Corti, and their possible relation to sensory transduction", Hearing research 15, 103-112. 18 
Poch-Broto, J., Carricondo, F., Bhathal, B., Iglesias, M.-C., López-Moya, J., Rodríguez, F., Sanjuán, J., and Gil-Loyzaga, P. (2009). "Cochlear microphonic audiometry: a new hearing test for objective diagnosis of deafness", Acta otolaryngologica 129, 749-754. 4, 24, 25, 27

Pozrikidis, C. (2008). "Boundary-integral modeling of cochlear hydrodynamics", Journal of Fluids and Structures 24, 336-365. 33

Pozrikidis, C. (2009). Fluid dynamics (Springer). 33

Probst, R., Lonsbury-Martiann, B. L., and Martin, G. K. (1991). "A review of otoacoustie emissions", The Journal of the Acoustical Society of America 89, 2027-2067. 6, 19, 87, 91, 99

Puria, S. (2003). "Measurements of human middle ear forward and reverse acoustics: implications for otoacoustic emissions", The Journal of the Acoustical Society of America 113, 2773. 96

Puria, S. and Allen, J. B. (1991). "A parametric study of cochlear input impedance", The Journal of the Acoustical Society of America 89, 287. 44

Quarles, T., Newton, A., Pederson, D., and Sangiovanni-Vincentelli, A. (1993). "SPICE3 version $3 \mathrm{f} 3$ users manual", University of California, Berkeley, CA . 127

Ramamoorthy, S., Deo, N. V., and Grosh, K. (2007). "A mechano-electroacoustical model for the cochlea: Response to acoustic stimuli”, The Journal of the Acoustical Society of America 121, 2758-2773. 37, 39, 40, 46, 132, 138

Ramsden, R. T. (2013). "History of cochlear implantation", Cochlear Implants International 14, S3-S5. 3

Rapson, M. J., Tapson, J. C., and Karpul, D. (2012). "Unification and extension of monolithic state space and iterative cochlear models", The Journal of the Acoustical Society of America 131, 3935. 88, 92, 101, 106, 119 
Rattay, F., Leao, R. N., and Felix, H. (2001). "A model of the electrically excited human cochlear neuron. ii. influence of the three-dimensional cochlear structure on neural excitability", Hearing research 153, 64-79. 132

Recio, A. and Rhode, W. S. (2000). "Basilar membrane responses to broadband stimuli", The Journal of the Acoustical Society of America 108, 2281-2298. 67

Recio, A., Rich, N. C., Narayan, S. S., and Ruggero, M. A. (1998). "Basilarmembrane responses to clicks at the base of the chinchilla cochlea", The Journal of the Acoustical Society of America 103, 1972. 66

Reddy, J. N. (2006). An introduction to the finite element method, volume 2 (McGraw-Hill New York). 109

Rhode, W. S. (1971). "Observations of the vibration of the basilar membrane in squirrel monkeys using the Mössbauer technique”, The Journal of the Acoustical Society of America 49, 1218. 30, 119

Rhode, W. S. (1984). "Cochlear mechanics”, Annual review of physiology 46, 231-246. 1, 30

Robles, L. and Ruggero, M. A. (2001). "Mechanics of the mammalian cochlea", Physiological reviews 81, 1305-1352. 1, 29, 61, 87, 106

Roush, P. (2008). "Auditory neuropathy spectrum disorder: Evaluation and management", The Hearing Journal 61, 36. 4

Ruggero, M. A. and Temchin, A. N. (2005). "Unexceptional sharpness of frequency tuning in the human cochlea", Proceedings of the National Academy of Sciences of the United States of America 102, 18614-18619. 61, 63

Russell, I. (2008). "Cochlear receptor potentials", in The senses: a comprehensive reference, volume 3:Audition, 320-358 (Elsevier). 4, 24, 36, 84

Salt, A. N. and Hullar, T. E. (2010). "Responses of the ear to low frequency sounds, infrasound and wind turbines", Hearing research 268, 12-21. 4, 25 
Salt, A. N. and Kaltenbach, J. A. (2011). "Infrasound from wind turbines could affect humans", Bulletin of Science, Technology \& Society 31, 296-302. 25

Salt, A. N., Lichtenhan, J. T., Gill, R. M., and Hartsock, J. J. (2013). "Large endolymphatic potentials from low-frequency and infrasonic tones in the guinea pig", The Journal of the Acoustical Society of America 133, 1561. 25

Santarelli, R., Scimemi, P., Dal Monte, E., and Arslan, E. (2006). "Cochlear microphonic potential recorded by transtympanic electrocochleography in normally-hearing and hearing-impaired ears", Acta otorhinolaryngologica italica 26, 78. 25

Saremi, A. and Stenfelt, S. (2013). "Effect of metabolic presbyacusis on cochlear responses: A simulation approach using a physiologically-based model", The Journal of the Acoustical Society of America 134, 2833-2851. 107

Senturia, S. (2001). Microsystem design, chapter 5 (Kluwer academic publishers Boston, MA). 119, 120

Shera, C. A. (2003). "Mammalian spontaneous otoacoustic emissions are amplitude-stabilized cochlear standing waves", The Journal of the Acoustical Society of America 114, 244. 20, 88, 99, 109

Shera, C. A. and Guinan, J. J. (2008). "Mechanisms of mammalian otoacoustic emission", in Active processes and otoacoustic emissions in hearing, edited by G. Manley and R. Fay, pp. 305-342 (Springer, New York). 87, 88, 99

Shi, W., Ji, F., Lan, L., Liang, S.-C., Ding, H.-N., Wang, H., Li, N., Li, Q., Li, X.-Q., and Wang, Q.-J. (2012). "Characteristics of cochlear microphonics in infants and young children with auditory neuropathy", Acta oto-laryngologica 132, 188-196. 27

Sisto, R., Moleti, A., Paternoster, N., Botti, T., and Bertaccini, D. (2010). "Different models of the active cochlea, and how to implement them in the 
state-space formalism", The Journal of the Acoustical Society of America 128, 1191-1202. 52, 65

Snow, J. B., Wackym, P. A., and Ballenger, J. J. (2009). Ballenger's Otorhinolaryngology Head and Neck Surgery, 17 edition (Pmph USA Limited). 16

Starr, A., Zeng, F., Michalewski, H., and Moser, T. (2008). "Perspectives on auditory neuropathy: Disorders of inner hair cell, auditory nerve, and their synapse", in The senses: a comprehensive reference, volume 3:Audition, 397412 (Elsevier, Canada). 25, 26

Steele, C. R. and Taber, L. A. (1979). "Comparison of wkb and finite difference calculations for a two-dimensional cochlear model", The Journal of the Acoustical Society of America 65, 1001. 51

Steele, C. R. and Zais, J. G. (1985). "Effect of coiling in a cochlear model", The Journal of the Acoustical Society of America 77, 1849-1852. 31

Strelioff, D. (1973). "A computer simulation of the generation and distribution of cochlear potentials", The Journal of the Acoustical Society of America 54, 620-9. 36, 38, 46, 47, 79, 132, 133, 138

Tasaki, I., Davis, H., and Eldredge, D. (1954). "Exploration of cochlear potentials in guinea pig with a microelectrode.", The Journal of the Acoustical Society of America 26, 765-773. 24

Tasaki, I., Davis, H., and Legouix, J. P. (1952). "The space-time pattern of the cochlear microphonics (guinea pig), as recorded by differential electrode", the Journal of the Acoustical Society of America 24, 502-519. 24

Tasaki, I. and Fernandez, C. (1952). "Modification of cochlear microphonics and action potentials by $\mathrm{kcl}$ solution and by direct currents", Journal Of Neurophysiology 15, 497-512. 24 
Teal, P., Lineton, B., and Elliott, S. (2011). "An electromechanical model for the cochlear microphonic", in AIP Conf. Proc, volume 1403, 652-657. 4, 65, 119

Thorne, M., Salt, A. N., DeMott, J. E., Henson, M. M., Henson, O., and Gewalt, S. L. (1999). "Cochlear fluid space dimensions for six species derived from reconstructions of three-dimensional magnetic resonance images", The Laryngoscope 109, 1661-1668. 79, 132, 133, 135

Tran, P., Wong, P., Sue, A., Li, Q., and Carter, P. (2013). "Influence of blood vessel conductivity in cochlear implant stimulation using a finite element head model", in Engineering in Medicine and Biology Society (EMBC), 2013 35th Annual International Conference of the IEEE, 5291-5294 (IEEE). 79, 132

Tyberghein, J. and Forrez, G. (1971). "Objective (era) and subjective (cor) audiometry in the infant", Acta Oto-Laryngologica 71, 249-252. 27

Vilfan, A. and Duke, T. (2008). "Frequency clustering in spontaneous otoacoustic emissions from a lizard's ear", Biophysical journal 95, 4622-4630. 20, 87

Vlasak, W. (2012). "Analyzing atoms using the spice computer program", Computing in Science \& Engineering 14, 98. 128

Von Békésy, G. and Wever, E. G. (1960). Experiments in hearing, volume 8 (McGraw-Hill New York). 29

Wangemann, P. (2006). "Supporting sensory transduction: cochlear fluid homeostasis and the endocochlear potential", The Journal of physiology 576, $11-21.11$

Watts, L. (1993). "Cochlear mechanics: Analysis and analog VLSI", Ph.D. thesis, California Institute of Technology. 10, 11, 12, 13, 14, 15, 17, 128

Webster, D. (1973). “Audition”, Handbook of perception III: biology of perceptual systems. Academic Pr., New York 449-482. 39 
Wegel, R. and Lane, C. (1924). "The auditory masking of one pure tone by another and its probable relation to the dynamics of the inner ear", Physical Review 23, 266. 119,120

Wen, B. (2006). "Modeling the nonlinear active cochlea: Mathematics and analog vlsi”, Ph.D. thesis, The University of Pennsylvania. 87, 128

Wever, E. and Bray, C. (1930a). "Action currents in the auditory nerve in response to acoustic stimulation”, Proc Natl Acad Sci USA 10, 344-350. 3, 24

Wever, E. and Bray, C. (1930b). "Present possibilities for auditory theory", The Psychological Review 37, 365-380. 24

Wilson, M. (2008). "Interferometry data challenge prevailing view of wave propagation in the cochlea", Physics Today 61, 26. 1

Withnell, R. H. (2001). "Brief report: the cochlear microphonic as an indication of outer hair cell function", Ear and hearing 22, 75-77. 25, 36, 108, 109

Wysocki, J. (1999). "Dimensions of the human vestibular and tympanic scalae", Hearing research 135, 39-46. 79, 132, 134, 135

Yamamura, K., Kuroki, W., Okuma, H., and Inoue, Y. (2005). "Path following circuits-spice-oriented numerical methods where formulas are described by circuits-", IEICE transactions on fundamentals of electronics, communications and computer sciences $\mathbf{8 8}, 825-831.66$

Zajic, G. and Schacht, J. (1987). "Comparison of isolated outer hair cells from five mammalian species”, Hearing research 26, 249-256. 135

Zhang, M. (2013). "Effects of stimulus intensity on low-frequency toneburst cochlear microphonic waveforms", Audiology Research 3, e3. 3 